\title{
Variational approximations for correlation functions in quantum field theories
}

\author{
Cécile Martin \\ Groupe de Physique Théorique, \\ Institut de Physique Nucléaire, \\ F-91406, Orsay Cedex, France*
}

\begin{abstract}
Applying the time-dependent variational principle of Balian and Vénéroni, we derive variational approximations for multi-time correlation functions in $\Phi^{4}$ field theory. We assume first that the initial state is given and characterized by a density operator equal to a Gaussian density matrix. Then, we study the more realistic situation where only a few expectation values are given at the initial time and we perform an optimization with respect to the initial state. We calculate explicitly the two-time correlation functions with two and four field operators at equilibrium in the symmetric phase.
\end{abstract}

*E-mail address : martinc@ipno.in2p3.fr 
Variational Correlation Functions 


\section{Contents}

\section{1-Introduction}

2-Variational approximation for two-time correlation functions : optimization of the dynamics

- 2-1 Definition of the generating functional for correlation functions

- 2-2 Variational evaluation of the generating functional

- 2-3 Choice of the variational spaces

- 2-4 Dynamical equations with sources

- 2-5 Expansion in powers of the sources

- 2-5a- TDHB equations for the expectation values

- 2-5b-Two-time correlation functions

\section{3-Optimization of the initial state}

- 3-1 Introduction

- 3-2 Variational spaces

- 3-3 Dynamical equations

- 3-4 Expansion in powers of the sources

- 3-5 Approximation for the two-time and two-point correlation function in the symmetric phase. The case in equilibrium. The fluctuation-dissipation theorem.

- 3-6 Two-time and four-point correlation function :

Relation between the response function $\Pi_{R}\left(\mathbf{q}^{2}, t^{\prime}, t^{\prime \prime}\right)$ and the four-point correlation function $\Sigma^{\Phi \Phi}$. The case of the symmetric phase. A perturbative resolution of the dynamical equations. The variational expression for the response function $\Pi_{R}\left(\mathbf{q}^{2}, t^{\prime}, t^{\prime \prime}\right)$ at the lowest order and at the first order in the loop term.

\section{4-Conclusion}

\section{Appendices}


- Appendix A : Characterization of a Gaussian state

- Appendix B : Expression of the von Neuman entropy for bosons in the HartreeBogoliubov approximation

- Appendix C : Parametrization of the product of two Gaussians

- Appendix D : Dynamical equations for the expectation values $\alpha_{d}, \Xi_{d}, \alpha_{a}, \Xi_{a}$

- Appendix E : Useful integrals for the resolution of the dynamical equations of section 3

- Appendix F : Solutions of the backward dynamical equations to first order 


\section{Introduction}

The description of the time evolution of a system with initial conditions which are out of equilibrium is one of the main challenges in quantum field theory at finite temperature. This problem appears in different contexts in cosmology or in particle physics. In inflationary models for the primordial universe, the problem is to describe the evolution of the inflaton scalar field in order to be abble to predict the duration of the inflationary phase. Another important problem is the prediction of the density fluctuations and its relation to the distribution of galaxies [1]. In particle physics, the experiments at the Brookhaven Relativistic Heavy Ion Collider (RHIC) or soon at the CERN Large Hadron Collider (LHC) may show a phase transition between a state where the quarks and the gluons are deconfined and chiral symmetry restored and the state of ordinary matter. The state of the matter formed after the collision is out of equilibrium and the study of the dynamics near the phase transition requires a nonperturbative method. The chiral phase transition can be studied in a model of scalar fields with an $\mathrm{O}(\mathrm{N})$ symmetry. The relaxation of inhomogeneous pions configurations formed during the collision (called disoriented chiral condensates or DCC) could be the signal of the chiral phase transition through a pions distribution with strong correlations [2]. In the Standard model, one is interested in the dynamical properties of the electroweak phase transition in order to describe the baryogenesis. The violation of the baryonic number, which is related to the desintegration of non-trivial topological field configurations, the sphalerons, is evaluated from a correlation function calculated at high temperature in a classical approximation [3.

The scale for the energy density involved in the primordial universe or the length scale which is important for the collective modes in Quantum Chromodynamics require non-perturbative approaches. Variational methods are therefore particularly adapted.

In the mean-field Hartree-Fock (HF) approximation for fermions or the HartreeBogoliubov (HB) approximation for bosons, one minimizes the free energy or, at zero temperature, the energy with a Gaussian variational ansatz for the density matrix or the pure state. For a time-dependent problem, the corresponding approximations (TDHF or TDHB) are obtained by minimizing an action also with a Gaussian variational ansatz. (At zero temperature, a time-dependent variational principle was already introduced by Dirac [4]. It was used by the authors of reference [5] in the context of quantum field theories .) These methods are well adapted to the calculation of expectation values. However, they are not suitable for multi-time correlation functions. In order to obtain approximations for these multi-time correlation functions, a variational principle was recently introduced in the context of non-relativistic fermions [6]. The action to minimize has for stationary value the generating functional for the correlation functions. This functional is a functional of 
the time-dependent sources coupled to the operators of interest. The action involves two types of variational objects, one $\mathcal{A}(t)$ related to the observables of interest and the other $\mathcal{D}(t)$ akin to a density matrix. The temporal evolution of $\mathcal{A}(t)$ is described by a backward equation while that of $\mathcal{D}(t)$ involves a time running forward. Let us notice that this doubling of the number of degrees of freedom with two temporal arrows appears already in the formalism of Schwinger and Keldysh for time-dependent problems at finite temperature [7]. (For a review of the different formalisms used at finite temperature, see reference [8]). By restricting the variational spaces for $\mathcal{A}(t)$ and $\mathcal{D}(t)$ to Gaussian operators, one obtains evolution equations which are coupled. The expansion at first order in powers of the sources of these equations (or at the second order for the generating functional ) provides approximations for the two-time correlation functions. In spite of the Gaussian character of the variational ansätze, the approximations obtained in this way go beyond the usual mean-field approximations and include correlations between particles. This comes from the fact that the variational principle of Balian and Vénéroni [6] has been precisely constructed to provide directly the quantity of interest, namely the generating functional.

Our aim is to generalize this formalism to quantum field theories. For simplicity, we will consider the case of a scalar field described by a $\Phi^{4}$ interaction in a Minkowski space. We will choose sources coupled to local operators such as $\Phi(\mathbf{x})$ or $\Pi(\mathbf{x})$ (written here in the Schrödinger representation) and to composite operators such as $\Phi(\mathbf{x}) \Phi(\mathbf{y})$ or $\Phi(\mathbf{x}) \Pi(\mathbf{y})$. The second derivative of the generating functional with respect to the sources will thus give two-time correlation functions which have up to four field operators.

In the first part of the paper, we will consider that the initial state is given and characterized by a density operator $D\left(t_{0}\right)$ equal to a Gaussian density matrix. We will write the action functional to be minimized (eqs. (2.14) and (2.15)). Then we will restrict our variational spaces to operators which are exponentials of quadratic and linear forms in the field operators $\Phi(\mathbf{x})$ and $\Pi(\mathbf{x})$ (called Gaussian operators). An adequate parametrization of the variational objects allows us to write the coupled dynamical equations in a compact form. We stress again the fact that the variational method we use introduces a doubling of the degrees of freedom. We obtain four coupled equations (two for the expectation values of one-field operator and two for the expectation values of two-field operators). Therefore we have twice as many dynamical equations as the usual mean-field approach. The expansion of these equations to lowest zero order in powers of the sources leads to a decoupling of these equations whose a half is identical to the time-dependent mean-field equations for the $\Phi^{4}$ theory written in reference [9]. We have showed in a previous paper that these dynamical equations can be put into a classical noncanonical Hamiltonian framework with a Poisson structure which is a generalization of the standard Poisson bracket [10]. At the lowest order we thus obtain an approximation for the single-time 
expectation values of one or two field operators. The expansion of the coupled equations to first order in powers of the sources provides an approximation for the two-time correlation functions with two, three or four field operators. Their evaluation proceeds in the following way : first one solves the mean-field TDHB equations, then one calculates the kernel which corresponds to small deviations arround the mean-field solution (which is analogous to the random phase approximation (RPA) kernel of reference [6]) and finally one solves a backward dynamical equation which involves the kernel calculated for the mean-field solution. These results have been obtained for the generating functional of correlation functions with time-ordered product of the field operators. We also give the corresponding expressions for the retarded Green functions. Indeed, these retarded Green functions appear when one studies the response of the system to a small external perturbation. A less complete version of this first part of the paper has been published in reference [11].

In section 2 , we have supposed that the initial state $D\left(t_{0}\right)$ was known. In practice, this is not the case. Only the expectation values of a few observables are known. In section 3 , we use the form of the variational principle introduced by Balian and Vénéroni which optimizes also the initial state [6]. The approximations obtained in this way for the twotime correlation functions include not only the correlations between particles originating from the dynamics but also those present in the initial state. One introduces again two variational objects which now evolve in imaginary time. We study the symmetric phase. We will consider the two-time function with two field operators $\Phi$ and the twotime function with four field operators $\Phi$. We will relate it to the standard polarization function $\Pi_{R}$. At equilibrium, in the symmetric phase, the case of the correlation function with two field operators is special since the approximations obtained with or without optimization with respect to the initial state coïncide. For the correlation function with four field operators, we will be obliged to combine the variational approximation with a perturbative approximation valid for small values of the coupling constant, by neglecting or taking into account at first order a loop term in the dynamical equations. However, the solutions remain nonperturbative since they depend on the self-consistent HB solution. At the lowest order, by neglecting the loop term, the two-time function with four field operators is simply related to the two-time function with two field operators according to the Wick theorem. At equilibrium, in the symmetric phase, we give to first order the expression for the two-time polarization functions with four field operators : the retarded Green function $\Pi_{R}\left(\mathbf{q}^{2}, t^{\prime}, t^{\prime \prime}\right)$ (eq. 4.133a) and the non-retarded Green function $\Pi\left(\mathbf{q}^{2}, t^{\prime}, t^{\prime \prime}\right)$ (eq. (3.144) ). We verify explicitly that these functions depend only on the time difference $t^{\prime}-t^{\prime \prime}$ and not on the initial time $t_{0}$. This is not the case for the nonretarded Green function if, like in the section 2, we optimize only the dynamics and not the initial state. We check also that our approximations for the two-time correlation functions 
satisfy the fluctuation-dissipation theorem. The last section contains some conclusions and perspectives for future work.

\section{Variational approximation for the two-time corre- lation functions : optimization of the dynamics}

\subsection{Definition of the generating functional for correlation func- tions}

We define the operator $A(J, t)$ which is a functional of the sources $J$ and a function of the time $t$ in the following way :

$$
\begin{aligned}
A(J, t) & =T \exp i \int_{t}^{\infty} d t^{\prime}\left\{\int d^{3} x J^{\Phi}\left(\mathbf{x}, t^{\prime}\right) \Phi^{H}\left(\mathbf{x}, t^{\prime}, t\right)+J^{\Pi}\left(\mathbf{x}, t^{\prime}\right) \Pi^{H}\left(\mathbf{x}, t^{\prime}, t\right)\right. \\
& +\int d^{3} x d^{3} y J^{\Phi \Pi}\left(\mathbf{x}, \mathbf{y}, t^{\prime}\right)\left(\Phi^{H}\left(\mathbf{x}, t^{\prime}, t\right) \Pi^{H}\left(\mathbf{y}, t^{\prime}, t\right)+\Pi^{H}\left(\mathbf{y}, t^{\prime}, t\right) \Phi^{H}\left(\mathbf{x}, t^{\prime}, t\right)\right) \\
& \left.+J^{\Phi \Phi}\left(\mathbf{x}, \mathbf{y}, t^{\prime}\right) \Phi^{H}\left(\mathbf{x}, t^{\prime}, t\right) \Phi^{H}\left(\mathbf{y}, t^{\prime}, t\right)+J^{\Pi \Pi}\left(\mathbf{x}, \mathbf{y}, t^{\prime}\right) \Pi^{H}\left(\mathbf{x}, t^{\prime}, t\right) \Pi^{H}\left(\mathbf{y}, t^{\prime}, t\right)\right\}
\end{aligned}
$$

where $T$ is the time-ordered product of operators and $\Phi^{H}\left(\mathbf{x}, t^{\prime}, t\right)$ and $\Pi^{H}\left(\mathbf{x}, t^{\prime}, t\right)$ are respectively the field and momentum operators defined in the Heisenberg representation. They satisfy the boundary condition : $\Phi^{H}(\mathbf{x}, t, t)=\Phi(\mathbf{x})$ and $\Pi^{H}(\mathbf{x}, t, t)=\Pi(\mathbf{x}), \Phi(\mathbf{x})$ and $\Pi(\mathbf{x})$ being the operators in the Schrödinger representation. We use the short hand notation

$$
A(J, t)=T \exp \left(i \int_{t}^{\infty} d t^{\prime} J_{j}\left(t^{\prime}\right) \mathcal{O}_{j}\left(t^{\prime}, t\right)\right)
$$

The sources $J_{j}\left(t^{\prime}\right)$ (which are local or depend on two space points) are turned on between $t^{\prime}=t$ and $t^{\prime}=+\infty$.

The functional $A(J, t)$ satisfies the equation

$$
\frac{d}{d t} A(J, t)=i[A(J, t), H]-i A(J, t) \sum_{j} J_{j}(t) \mathcal{O}_{j}
$$

with the boundary condition $A(t=+\infty)=1$. We will consider the case of a selfinteracting scalar field in a Minkowski metric described by the Hamiltonian $H=\int d^{3} x \mathcal{H}(\mathbf{x})$ with :

$$
\mathcal{H}(\mathbf{x})=\frac{1}{2} \Pi^{2}(\mathbf{x})+\frac{1}{2}[\vec{\nabla} \Phi(\mathbf{x})]^{2}+\frac{m_{0}^{2}}{2} \Phi^{2}(\mathbf{x})+\frac{b}{4 !} \Phi^{4}(\mathbf{x}) .
$$

We work in three spatial dimensions. The constants $m_{0}$ and $b$ are respectively the bare mass and the bare coupling constant.

The generating functional for the causal Green functions writes

$$
Z\left(J, t_{0}\right)=\operatorname{Tr}\left(D\left(t_{0}\right) A\left(J, t_{0}\right)\right),
$$


$D\left(t_{0}\right)$ being the initial state. The statistical operator $D\left(t_{0}\right)$ may represent a thermal equilibrium or a nonequilibrium state. At zero temperature, it reduces to a projection operator. In addition to the sources $J$, the generating functional $Z$ depends on the initial time $t_{0}$. This is a difference with the generating functional usually considered in quantum field theories [12]. We want to evaluate the functional derivative of

$$
W\left(J, t_{0}\right)=-i \ln Z\left(J, t_{0}\right)
$$

$W\left(J, t_{0}\right)$ is the generating functional for the connected Green functions. Let us note that for hermitic operators $A(t)$ and $D(t), W(J, t)$ is real. The expansion in powers of the sources of $W(J, t)$ writes :

$$
\begin{aligned}
& W\left(J, t_{0}\right)=-i \ln \left(n_{0}\right)+\int_{t_{0}}^{+\infty} d t^{\prime}\left\{\int d^{3} x J_{\Phi}\left(\mathbf{x}, t^{\prime}\right) \varphi\left(\mathbf{x}, t^{\prime}\right)+\int d^{3} x d^{3} y J_{\Phi \Phi}\left(\mathbf{x}, \mathbf{y}, t^{\prime}\right) G\left(\mathbf{x}, \mathbf{y}, t^{\prime}\right)+\ldots\right\} \\
& +\frac{i}{2} \iint_{t_{0}}^{+\infty} d t^{\prime} d t^{\prime \prime}\left\{\int d^{3} x_{1} d^{3} x_{2} J_{\Phi}\left(\mathbf{x}_{1}, t^{\prime}\right) J_{\Phi}\left(\mathbf{x}_{2}, t^{\prime \prime}\right) C_{\Phi \Phi}^{2}\left(\mathbf{x}_{1}, \mathbf{x}_{2}, t^{\prime}, t^{\prime \prime}\right)+\ldots\right\} \\
& +\ldots
\end{aligned}
$$

where $n_{0}=\operatorname{Tr} D\left(t_{0}\right)$ ( we choose not to normalize the operator $D\left(t_{0}\right)$ ). We introduce the following notations for the expectation values of one and two field operators :

$$
\begin{gathered}
\varphi(\mathbf{x}, t)=\frac{1}{n_{0}} \operatorname{Tr}\left(\Phi^{H}\left(\mathbf{x}, t, t_{0}\right) D\left(t_{0}\right)\right) \\
G(\mathbf{x}, \mathbf{y}, t)=\frac{1}{n_{0}} \operatorname{Tr}\left(\Phi^{H}\left(\mathbf{x}, t, t_{0}\right) \Phi^{H}\left(\mathbf{y}, t, t_{0}\right) D\left(t_{0}\right)\right)-\varphi(\mathbf{x}, t) \varphi(\mathbf{y}, t) .
\end{gathered}
$$

The two-time causal functions with two field operators are defined according to :

$$
C_{\Phi \Phi}^{2}\left(\mathbf{x}, \mathbf{y}, t^{\prime}, t^{\prime \prime}\right)=\frac{1}{n_{0}} \operatorname{Tr}\left(T \Phi^{H}\left(\mathbf{x}, t^{\prime}, t_{0}\right) \Phi^{H}\left(\mathbf{y}, t^{\prime \prime}, t_{0}\right) D\left(t_{0}\right)\right)-\varphi\left(\mathbf{x}, t^{\prime}\right) \varphi\left(\mathbf{y}, t^{\prime \prime}\right) .
$$

Similarly, we define the functions $C_{\Phi \Pi}^{2}, C_{\Pi \Pi}^{2}$. From the expansion (2.7), we have :

$$
\left.\frac{1}{i} \frac{\delta^{2} W}{\delta J_{\Phi}\left(\mathbf{x}, t^{\prime}\right) \delta J_{\Phi}\left(\mathbf{y}, t^{\prime \prime}\right)}\right|_{J=0}=C_{\Phi \Phi}^{2}\left(\mathbf{x}, \mathbf{y}, t^{\prime}, t^{\prime \prime}\right) .
$$

We define also the three-point and four point two-time correlation function $C^{3}$ et $C^{4}$ :

$$
\begin{aligned}
& \left.\frac{1}{i} \quad \frac{\delta^{2} W}{\delta J_{\Phi}\left(\mathbf{x}, t^{\prime}\right) \delta J_{\Phi \Phi}\left(\mathbf{y}, \mathbf{z}, t^{\prime \prime}\right)}\right|_{J=0} \equiv C^{3}\left(\mathbf{x}, \mathbf{y}, \mathbf{z}, t^{\prime}, t^{\prime \prime}\right) \\
& +\varphi\left(\mathbf{y}, t^{\prime \prime}\right)\left(C^{2}\left(\mathbf{x}, \mathbf{z}, t^{\prime}, t^{\prime \prime}\right)+\varphi\left(\mathbf{x}, t^{\prime}\right) \varphi\left(\mathbf{z}, t^{\prime \prime}\right)\right)+\varphi\left(\mathbf{z}, t^{\prime \prime}\right)\left(C^{2}\left(\mathbf{x}, \mathbf{y}, t^{\prime}, t^{\prime \prime}\right)+\varphi\left(\mathbf{x}, t^{\prime}\right) \varphi\left(\mathbf{y}, t^{\prime \prime}\right)\right) \\
& \left.\frac{\delta^{2} W}{i} \quad \frac{(2.12)}{\delta J_{\Phi \Phi}\left(\mathbf{x}, \mathbf{y}, t^{\prime}\right) \delta J_{\Phi \Phi}\left(\mathbf{z}, \mathbf{u}, t^{\prime \prime}\right)}\right|_{J=0} \equiv C^{4}\left(\mathbf{x}, \mathbf{y}, \mathbf{z}, \mathbf{u}, t^{\prime}, t^{\prime \prime}\right) \\
& \quad+\left(C^{2}\left(\mathbf{x}, \mathbf{z}, t^{\prime}, t^{\prime \prime}\right)+\varphi\left(\mathbf{x}, t^{\prime}\right) \varphi\left(\mathbf{z}, t^{\prime \prime}\right)\right)\left(C^{2}\left(\mathbf{y}, \mathbf{u}, t^{\prime}, t^{\prime \prime}\right)+\varphi\left(\mathbf{y}, t^{\prime}\right) \varphi\left(\mathbf{u}, t^{\prime \prime}\right)\right) \\
& \quad+\left(C^{2}\left(\mathbf{x}, \mathbf{u}, t^{\prime}, t^{\prime \prime}\right)+\varphi\left(\mathbf{x}, t^{\prime}\right) \varphi\left(\mathbf{u}, t^{\prime \prime}\right)\right)\left(C^{2}\left(\mathbf{y}, \mathbf{z}, t^{\prime}, t^{\prime \prime}\right)+\varphi\left(\mathbf{y}, t^{\prime}\right) \varphi\left(\mathbf{z}, t^{\prime \prime}\right)\right)
\end{aligned}
$$




\subsection{Variational evaluation of the generating functional}

We will use the time-dependent variational principle of Balian and Vénéroni [6] to obtain an approximation for our quantity of interest $\operatorname{Tr}\left(D\left(t_{0}\right) A\left(J, t_{0}\right)\right)$. This principle is an illustration of the general method developped in reference 13 to construct a variational principle suited to the evaluation of a desired quantity. The basic idea is to consider the equation (2.3) which defines $A\left(J, t_{0}\right)$ as a constraint and to introduce a lagrangian multiplier $\mathcal{D}\left(t_{0}\right)$ associated to this constraint.

We define the action functional :

$$
\mathcal{Z}(\mathcal{A}(t), \mathcal{D}(t))=\operatorname{Tr}\left(\mathcal{A}\left(t_{0}\right) \mathcal{D}\left(t_{0}\right)\right)+\mathcal{Z}_{\text {dyn }}
$$

where

$$
\mathcal{Z}_{\text {dyn }}=\operatorname{Tr} \int_{t_{0}}^{\infty} d t \mathcal{D}(t)\left\{\frac{d \mathcal{A}(t)}{d t}-i\left[\mathcal{A}(t), \int d^{3} x \mathcal{H}(\mathbf{x})\right]+i \mathcal{A}(t)\left(\sum_{j} J_{j}(t) Q_{j}\right)\right\} .
$$

We have written in a compact form the term which involves the sources $J$ :

$$
\begin{aligned}
\sum_{j} J_{j}(t) Q_{j}= & \int d^{3} x\left(J^{\Phi}(\mathbf{x}, t) \Phi(\mathbf{x})+J^{\Pi}(\mathbf{x}, t) \Pi(\mathbf{x})\right) \\
& +\int d^{3} x d^{3} y J^{\Phi \Pi}(\mathbf{x}, \mathbf{y}, t)(\Phi(\mathbf{x}) \Pi(\mathbf{y})+\Pi(\mathbf{y}) \Phi(\mathbf{x})) \\
& +\int d^{3} x d^{3} y\left(J^{\Phi \Phi}(\mathbf{x}, \mathbf{y}, t) \Phi(\mathbf{x}) \Phi(\mathbf{y})+J^{\Pi \Pi}(\mathbf{x}, \mathbf{y}, t) \Pi(\mathbf{x}) \Pi(\mathbf{y})\right)
\end{aligned}
$$

The variational quantities of the action functional $\mathcal{Z}$ are the observable-like operator $\mathcal{A}(t)$ and the density-like operator $\mathcal{D}(t)$. We look for the stationarity of $\mathcal{Z}$ with respect to variations of $\mathcal{A}(t)$ and $\mathcal{D}(t)$ with the boundary conditions : $\mathcal{A}(t=+\infty)=A(t=+\infty)=1$ and $\mathcal{D}\left(t_{0}\right)=D\left(t_{0}\right)$. $D\left(t_{0}\right)$ is the initial statistical operator which we assume to be given and equal to a Gaussian density matrix. By construction of the variational principle, the generating functional for the connected Green functions will be approximated by the value of the functional $\mathcal{Z}$ at the stationary point :

$$
W\left(J, t_{0}\right)=-i \ln \mathcal{Z}_{s t}
$$

\subsection{Choice of the variational spaces}

We restrict ourselves to trial operators $\mathcal{A}(t)$ and $\mathcal{D}(t)$ which are exponentials of quadratic and linear forms of the field operators $\Phi(\mathbf{x})$ and $\Pi(\mathbf{x})$ which we shall loosely call Gaussian operators.

A Gaussian state $\mathcal{D}(t)$ is completely characterized by the vector $\alpha(\mathbf{x}, t)$ and the matrix $\Xi(\mathbf{x}, \mathbf{y}, t)$ defined by :

$$
\alpha(\mathbf{x}, t)=\left(\begin{array}{c}
\varphi(\mathbf{x}, t) \\
-i \pi(\mathbf{x}, t)
\end{array}\right)
$$




$$
\Xi(\mathbf{x}, \mathbf{y}, t)=\left(\begin{array}{cc}
2 G(\mathbf{x}, \mathbf{y}, t) & -i T(\mathbf{x}, \mathbf{y}, t) \\
-i T(\mathbf{y}, \mathbf{x}, t) & -2 S(\mathbf{x}, \mathbf{y}, t)
\end{array}\right)
$$

where

$$
\begin{gathered}
\varphi(\mathbf{x}, t)=\frac{1}{n(t)} \operatorname{Tr}(\mathcal{D}(t) \Phi(\mathbf{x})) \\
\pi(\mathbf{x}, t)=\frac{1}{n(t)} \operatorname{Tr}(\mathcal{D}(t) \Pi(\mathbf{x})) \\
G(\mathbf{x}, \mathbf{y}, t)=\frac{1}{n(t)} \operatorname{Tr}(\mathcal{D}(t) \bar{\Phi}(\mathbf{x}) \bar{\Phi}(\mathbf{y})) \\
S(\mathbf{x}, \mathbf{y}, t)=\frac{1}{n(t)} \operatorname{Tr}(\mathcal{D}(t) \bar{\Pi}(\mathbf{x}) \bar{\Pi}(\mathbf{y})) \\
T(\mathbf{x}, \mathbf{y}, t)=\frac{1}{n(t)} \operatorname{Tr}(\mathcal{D}(t)(\bar{\Phi}(\mathbf{x}) \bar{\Pi}(\mathbf{y})+\bar{\Pi}(\mathbf{y}) \bar{\Phi}(\mathbf{x}))) \\
n(t)=\operatorname{Tr} \mathcal{D}(t)
\end{gathered}
$$

and, for any operator $O: \bar{O}=O-\operatorname{Tr}(\mathcal{D}(t) O)$. The hermiticity of $\mathcal{D}(t)$ implies that the matrices $G, S$ and $T$ are real and that $G$ and $S$ are symmetric in the space indices.

The Heisenberg invariant is equal to :

$$
\begin{aligned}
& I(\mathbf{x}, \mathbf{y})=\int d^{3} z \quad(4<\bar{\Phi}(\mathbf{x}) \bar{\Phi}(\mathbf{z})><\bar{\Pi}(\mathbf{z}) \bar{\Pi}(\mathbf{y})> \\
&-<\bar{\Phi}(\mathbf{x}) \bar{\Pi}(\mathbf{z})+\bar{\Pi}(\mathbf{z}) \bar{\Phi}(\mathbf{x})><\bar{\Phi}(\mathbf{z}) \bar{\Pi}(\mathbf{y})+\bar{\Pi}(\mathbf{y}) \bar{\Phi}(\mathbf{z})>) \\
& I(\mathbf{x}, \mathbf{y})=\int d^{3} z\left(-\Xi_{11}(\mathbf{x}, \mathbf{z}) \Xi_{22}(\mathbf{z}, \mathbf{y})+\Xi_{12}(\mathbf{x}, \mathbf{z}) \Xi_{12}(\mathbf{z}, \mathbf{y})\right) .
\end{aligned}
$$

For a pure state :

$$
\begin{gathered}
\Xi_{11} \Xi_{22}-\Xi_{12}^{2}+1=0 \\
I(\mathbf{x}, \mathbf{y})=\delta^{3}(\mathbf{x}-\mathbf{y})
\end{gathered}
$$

In Appendix A, we give the relations between our parametrization of a Gaussian state and the parametrization used by the authors of reference [9].

We then introduce the operators $\mathcal{T}_{b}=\mathcal{A}(t) \mathcal{D}(t)$ and $\mathcal{T}_{c}=\mathcal{D}(t) \mathcal{A}(t)$ [14. The operator $\mathcal{T}_{b}$ is a Gaussian operator which is characterized by the following expectation values :

$$
\begin{gathered}
n(t)=\operatorname{Tr} \mathcal{T}_{b}=\operatorname{Tr} \mathcal{T}_{c} \\
\varphi_{b}(\mathbf{x}, t)=\frac{1}{n(t)} \operatorname{Tr}\left(\mathcal{T}_{b} \Phi(\mathbf{x})\right) \\
\pi_{b}(\mathbf{x}, t)=\frac{1}{n(t)} \operatorname{Tr}\left(\mathcal{T}_{b} \Pi(\mathbf{x})\right) \\
G_{b}(\mathbf{x}, \mathbf{y}, t)=\frac{1}{n(t)} \operatorname{Tr}\left(\mathcal{T}_{b} \bar{\Phi}(\mathbf{x}) \bar{\Phi}(\mathbf{y})\right)
\end{gathered}
$$




$$
\begin{gathered}
S_{b}(\mathbf{x}, \mathbf{y}, t)=\frac{1}{n(t)} \operatorname{Tr}\left(\mathcal{T}_{b} \bar{\Pi}(\mathbf{x}) \bar{\Pi}(\mathbf{y})\right) \\
T_{b}(\mathbf{x}, \mathbf{y}, t)=\frac{1}{n(t)} \operatorname{Tr}\left(\mathcal{T}_{b}(\bar{\Phi}(\mathbf{x}) \bar{\Pi}(\mathbf{y})+\bar{\Pi}(\mathbf{y}) \bar{\Phi}(\mathbf{x}))\right)
\end{gathered}
$$

with $\bar{\Phi}(\mathbf{x})=\Phi(\mathbf{x})-\varphi_{b}(\mathbf{x}, t)$ and $\bar{\Pi}(\mathbf{x})=\Pi(\mathbf{x})-\pi_{b}(\mathbf{x}, t)$.

Similarly the operator $\mathcal{T}_{c}$ is a Gaussian operator which is characterized by the quantities $n, \varphi_{c}, \pi_{c}, G_{c}, S_{c}, T_{b}$ ( obtained by replacing $\mathcal{T}_{b}$ by $\mathcal{T}_{c}$ in (2.31)-(2.35)). For $\mathcal{D}(t)=1$, one has $\varphi_{b}(\mathbf{x}, t)=\varphi_{c}(\mathbf{x}, t)=\varphi_{a}(\mathbf{x}, t)$ (and similar definitions for $\left.\pi_{a}, G_{a}, S_{a}, T_{a}\right)$. For $\mathcal{A}(t)=1$, one has $\varphi_{b}(\mathbf{x}, t)=\varphi_{c}(\mathbf{x}, t)=\varphi_{d}(\mathbf{x}, t)$ (and similar definitions for $\pi_{d}, G_{d}, S_{d}, T_{d}$ ). In Appendix $\mathrm{C}$, we give the expressions of $\alpha_{b}, \alpha_{c}, \Xi_{b}$ and $\Xi_{c}$ as functions of the independent variational quantities $\alpha_{a}, \Xi_{a}$ and $\alpha_{d}, \Xi_{d}$ which characterize respectively $\mathcal{A}$ and $\mathcal{D}$.

With this choice of the trial spaces for $\mathcal{A}(t)$ and $\mathcal{D}(t)$, the Wick theorem allows us to express the functional $\mathcal{Z}$ in the form :

$$
\begin{aligned}
\mathcal{Z}= & n\left(t_{0}\right)+\int_{t_{0}}^{+\infty} d t\left[\left.\frac{d n}{d t}\right|_{\mathcal{D}(t)=c t e}-i n(t) \int d^{3} x\left(\mathcal{E}_{c}(\mathbf{x}, t)-\mathcal{E}_{b}(\mathbf{x}, t)\right),\right. \\
& \left.+i n(t) \int d^{3} x_{1} d^{3} x_{2} \mathcal{K}_{c}\left(\mathbf{x}_{1}, \mathbf{x}_{2}, t\right)\right]
\end{aligned}
$$

where the energy density $\mathcal{E}(\mathbf{x}, t)=\operatorname{Tr}(\mathcal{D}(t) \mathcal{H}(\mathbf{x}, t))$ is given, for the Hamiltonian density (2.4), by :

$$
\begin{aligned}
\mathcal{E}(\mathbf{x}, t)= & \frac{1}{2} \pi^{2}(\mathbf{x}, t)+\frac{1}{2}(\vec{\nabla} \varphi(\mathbf{x}, t))^{2}+\frac{m_{0}^{2}}{2} \varphi^{2}(\mathbf{x}, t)+\frac{b}{24} \varphi^{4}(\mathbf{x}, t) \\
& +\frac{1}{2} S(\mathbf{x}, \mathbf{x}, t)-\left.\frac{1}{2} \Delta G(\mathbf{x}, \mathbf{y}, t)\right|_{\mathbf{x}=\mathbf{y}}+\frac{m_{0}^{2}}{2} G(\mathbf{x}, \mathbf{x}, t) \\
& +\frac{b}{8} G^{2}(\mathbf{x}, \mathbf{x}, t)+\frac{b}{4} \varphi^{2}(\mathbf{x}, t) G(\mathbf{x}, \mathbf{x}, t)
\end{aligned}
$$

and

$$
\begin{aligned}
\ln n(t)= & \ln n_{a}+\ln n_{d}-\frac{1}{2} \tilde{\alpha}_{b-c} \tau \alpha_{a-d} \\
& +\frac{1}{2} \ln \operatorname{det}\left(\sigma_{1}(1+\tau)\left(\Xi_{a}+\Xi_{d}\right)^{-1}(1+\tau)\right),
\end{aligned}
$$

where $\alpha_{b-c}=\alpha_{b}-\alpha_{c}$ and $\tau$ and $\sigma_{1}$ are the following $2 \times 2$ matrices :

$$
\sigma_{1}=\left(\begin{array}{ll}
0 & 1 \\
1 & 0
\end{array}\right) \quad, \quad \tau=\left(\begin{array}{cc}
0 & 1 \\
-1 & 0
\end{array}\right) .
$$

The last term of the functional (2.36), $K_{c}=\operatorname{Tr}\left(\mathcal{T}_{c} \sum_{j} J_{j}(t) Q_{j}\right)=\int d^{3} x_{1} d^{3} x_{2} \mathcal{K}_{c}\left(\mathbf{x}_{1}, \mathbf{x}_{2}, t\right)$, involves the sources $J$ and the expectation values indexed by $c$.

With the parametrization we use, the boundary conditions $\mathcal{D}\left(t_{0}\right)=D\left(t_{0}\right)$ and $\mathcal{A}(t=+\infty)=1$ correspond respectively :

$$
n_{d}\left(t_{0}\right)=n_{0}, \alpha_{d}\left(t_{0}\right)=\alpha_{0}, \Xi_{d}\left(t_{0}\right)=\Xi_{0},
$$


where $n_{0}, \alpha_{0}$ and $\Xi_{0}$ characterize the Gaussian initial state $D\left(t_{0}\right)$, and

$$
n_{a}(t=+\infty)=1, \Xi_{a}^{-1}(t=+\infty)=0, \frac{1}{\Xi_{a}} \alpha_{a}(t=+\infty)=0 .
$$

We will write the conditions for the stationarity of the functional $\mathcal{Z}$ with respect to the variations of $n_{d}, \alpha_{d}, \Xi_{d}$ and $n_{a}, \alpha_{a}, \Xi_{a}$.

\subsection{Dynamical equations with sources}

By varying the expression (2.36) with respect to $n_{d}, \alpha_{d}, \Xi_{d}$, with the boundary conditions

$$
n_{d}\left(t_{0}\right)=n_{0}, \alpha_{d}\left(t_{0}\right)=\alpha_{0}, \Xi_{d}\left(t_{0}\right)=\Xi_{0}
$$

we obtain the evolution equation for $n_{a}, \alpha_{a}$ and $\Xi_{a}$. Integrating (2.36) by parts and varying with respect to $n_{a}, \alpha_{a}, \Xi_{a}$ with the boundary conditions

$$
n_{a}(t=+\infty)=1, \Xi_{a}^{-1}(t=+\infty)=0, \frac{1}{\Xi_{a}} \alpha_{a}(t=+\infty)=0
$$

we obtain the evolution equations for $n_{d}, \alpha_{d}$ and $\Xi_{d}$. In general the evolution equations for $n_{d}, \alpha_{d}, \Xi_{d}$ and those for $n_{a}, \alpha_{a}, \Xi_{a}$ are coupled. The solutions $n_{d}, \alpha_{d}, \Xi_{d}$ and $n_{a}, \alpha_{a}, \Xi_{a}$ depend on the sources. Owing to this dependence, we will be abble to obtain non trivial approximations for the correlation functions in spite of the Gaussian nature of the trial operators.

By combining the evolution equation for $n_{d}, \alpha_{d}, \Xi_{d}$ with those for $n_{a}, \alpha_{a}, \Xi_{a}$, the dynamical equations for the expectation values $\alpha_{b}, \Xi_{b}$ and $\alpha_{c}, \Xi_{c}$ can be written in the following compact form :

$$
\begin{gathered}
i \dot{\alpha}_{b}=\tau w_{b}, \\
i \dot{\alpha}_{c}=\tau\left(w_{c}-w_{K}^{c}\right), \\
i \dot{\Xi}_{b}=2\left(\Xi_{b} \mathcal{H}_{b} \tau-\tau \mathcal{H}_{b} \Xi_{b}\right) \\
i \dot{\Xi}_{c}=2\left(\Xi_{c}\left(\mathcal{H}_{c}-\mathcal{I}_{K}^{c}\right) \tau-\tau\left(\mathcal{H}_{c}-\mathcal{I}_{K}^{c}\right) \Xi_{c}\right) .
\end{gathered}
$$

We have also $\dot{n}(t)=0$.

The vector $w_{b}$ and the matrix $\mathcal{H}_{b}$ are defined from the variation of $<H>_{b}=$ $\operatorname{Tr}\left(\mathcal{T}_{b} H\right):$

$$
\delta<H>_{b}=\int d^{3} x \tilde{w}_{b}(\mathbf{x}, t) \delta \alpha_{b}(\mathbf{x}, t)-\frac{1}{2} \int d^{3} x d^{3} y \operatorname{tr}\left[\mathcal{H}_{b}(\mathbf{x}, \mathbf{y}, t) \delta \Xi_{b}(\mathbf{y}, \mathbf{x}, t)\right] .
$$

Similarly, $w_{c}, \mathcal{H}_{c}$ and $w_{K}^{c}, \mathcal{I}_{K}^{c}$ are defined from the variation of $<H>_{c}=\operatorname{Tr}\left(\mathcal{T}_{c} H\right)$ and from the variation of the source term $K_{c}$. 
Therefore, by using a convenient parametrization for the two variational objects $\mathcal{D}(t)$ and $\mathcal{A}(t)$, we have been abble to obtain dynamical equations in a very compact form even for finite values of the sources. The dynamical equations for $\alpha_{d}, \Xi_{d}$ and $\alpha_{a}, \Xi_{a}$ have a more complicated form. They are given in Appendix D where we give also the explicit expressions of the vectors $w, w_{K}^{c}$ and of the matrices $\mathcal{H}$ and $\mathcal{I}_{K}^{c}$ in the case of the $\Phi^{4}$ theory. In spite of their simple form, the equations (2.44)-(2.47) are coupled because the solutions $\alpha_{b}, \Xi_{b}$ and $\alpha_{c}, \Xi_{c}$ do not satisfy simple boundary conditions.

Let us note that the coupled equations for $\alpha_{d}, \Xi_{d}\left(\alpha_{a}, \Xi_{a}\right)$ do not conserve neither the energy $<H>_{d}\left(<H>_{a}\right)$ nor the entropy $S_{d}\left(S_{a}\right)$ (see Appendix B for the definition of the von-Neuman entropy for bosons). On the other hand, the dynamical equations for $\alpha_{b}$ and $\Xi_{b}$ (eqs. (2.44) and (2.46) ) which have the same form as the mean-field TDHB equations conserve the pseudo-energy $<H>_{b}$ and the pseudo-entropy $S_{b}$.

The expansion in powers of the sources of the stationarity conditions (2.44)- (2.47) will provide approximate dynamical equations for the expectation values and the correlation functions defined in Eqs. (2.8)-(2.13).

\subsection{Expansion in powers of the sources}

\subsubsection{TDHB equations for the expectation values}

We will use the upper index ${ }^{(0)}$ for the solutions of the dynamical equations when there are no sources. The limit with vanishing sources corresponds to

$$
\alpha_{b}^{(0)}=\alpha_{c}^{(0)}=\alpha_{d}^{(0)} \quad, \quad \Xi_{b}^{(0)}=\Xi_{c}^{(0)}=\Xi_{d}^{(0)}
$$

From equations (C.4) and (C.5) and from

$$
\alpha_{b}-\alpha_{c}=2 \tau \frac{1}{\Xi_{a}+\Xi_{d}}\left(\alpha_{a}-\alpha_{d}\right),
$$

we obtain that in the limit with vanishing sources

$$
\Xi_{a}^{-1}=0 \quad, \quad \frac{1}{\Xi_{a}} \alpha_{a}=0
$$

We have also $w_{b}{ }^{(0)}=w_{c}{ }^{(0)}=w^{(0)}$ and $\mathcal{H}_{b}^{(0)}=\mathcal{H}_{c}{ }^{(0)}=\mathcal{H}^{(0)}$. In this limit, the dynamical equations (D.2) and (D.3) for $\alpha_{d}$ and $\Xi_{d}$ become (we suppress the index d) :

$$
\begin{gathered}
i \dot{\alpha}^{(0)}=\tau w^{(0)}, \\
i \dot{\Xi}^{(0)}=-\left[\left(\Xi^{(0)}+\tau\right) \mathcal{H}^{(0)}\left(\Xi^{(0)}-\tau\right)-\left(\Xi^{(0)}-\tau\right) \mathcal{H}^{(0)}\left(\Xi^{(0)}+\tau\right)\right] .
\end{gathered}
$$


These equations are the analog for the $\Phi^{4}$ theory of the time-dependent Hartree-Bogoliubov (TDHB) equations in nonrelativistic physics. They are equivalent to the dynamical equations obtained in reference [9] where the authors used an alternative form of the BalianVénéroni variational principle suited to the evaluation of single-time expectation values 15.

In reference [10], we have shown that these TDHB equations for the $\Phi^{4}$ theory can be written as classical dynamical equations with a nonsymplectic Poisson structure with the free-energy density playing the role of a classical Hamiltonian. The Heisenberg invariant (2.26) appears as a structural invariant of the Poisson structure.

The static HB solution satisfies $w_{0}=0$ that is, in the uniform case :

$$
\left(m_{0}^{2}+\frac{b}{6} \varphi_{0}+\frac{b}{2} G_{0}(\mathbf{x}, \mathbf{x})\right) \varphi_{0}=0
$$

and the so-called gap

$$
\Xi_{0}=-\tau \operatorname{coth}\left(\beta \mathcal{H}_{0} \tau\right)
$$

which can also be written as :

$$
\beta \mathcal{H}_{0}=\frac{1}{2} \tau \ln \left(\frac{\Xi_{0}+\tau}{\Xi_{0}-\tau}\right)
$$

More explicitly, in the uniform case, by going to the momentum space :

$$
\Xi_{0}(\mathbf{p})=\left(\begin{array}{cc}
\frac{1}{\omega_{\mathbf{p}}} \operatorname{coth} \frac{\beta \omega_{\mathbf{p}}}{2} & 0 \\
0 & -\omega_{\mathbf{p}} \operatorname{coth} \frac{\beta \omega_{\mathbf{p}}}{2}
\end{array}\right)
$$

and for the $\Phi^{4}$ theory, $\omega_{\mathbf{p}}=\sqrt{-g_{0}(\mathbf{p})}$ with

$$
g_{0}(\mathbf{p})=-\left(\mathbf{p}^{2}+m_{0}^{2}+\frac{b}{2} \varphi_{0}^{2}+\frac{b}{2} G_{0}(\mathbf{x}, \mathbf{x})\right) .
$$

The solution $\varphi_{0}=0$ of equation (2.54) corresponds to the symmetric phase while the solution $\varphi_{0} \neq 0$ corresponds to the broken phase.

We define the self-consistent mass $m(\beta)$ depending on the temperature as the solution of the gap equation :

$$
m^{2}(\beta)=m_{0}^{2}+\frac{b}{2} \varphi_{0}^{2}+\frac{b}{2} G_{0}(\mathbf{x}, \mathbf{x})
$$

By using eq. (2.54), we have also :

$$
m^{2}(\beta)=c\left(m_{0}^{2}+\frac{b}{2} G_{0}(\mathbf{x}, \mathbf{x})\right)
$$

with $c=1$ in the symmetric phase and $c=-2$ in the broken phase. 
The gap equation (2.59) has ultraviolet divergences and requires a regularization. The divergent part $G_{0}^{\text {div }}(\mathbf{x}, \mathbf{x})$ of $G_{0}(\mathbf{x}, \mathbf{x})$ comes from the zero temperature contribution. By introducing a cutoff $\Lambda$ in momentum,

$$
G_{0}^{\mathrm{div}}(\mathbf{x}, \mathbf{x})=\frac{1}{8 \pi^{2}}\left[\Lambda^{2}-\bar{m}^{2} \log \left(\frac{2 \Lambda}{\sqrt{e} \bar{m}}\right)\right]
$$

where $\bar{m}=m(T=0)$. The gap equation can be put in a finite form by choosing the following renormalization conditions [16] :

$$
\begin{gathered}
\epsilon \mu^{2}=\frac{m_{0}^{2}+\frac{b \Lambda^{2}}{16 \pi^{2}}}{\frac{1}{c}+\frac{b}{16 \pi^{2}} \log \left(\frac{2 \Lambda}{\sqrt{e} \mu}\right)}, \epsilon=0, \pm 1 \\
\frac{1}{g_{R}(\mu)}=\frac{2}{c b}+\frac{1}{8 \pi^{2}} \log \left(\frac{2 \Lambda}{\sqrt{e} \mu}\right)
\end{gathered}
$$

where $\mu$ is an arbitrary mass scale. The renormalized gap equation writes at zero temperature :

$$
\bar{m}^{2}=\epsilon \mu^{2}+\frac{g_{R}(\mu)}{16 \pi^{2}} \bar{m}^{2} \log \left(\frac{\bar{m}^{2}}{\mu^{2}}\right)
$$

In the next sections, the dynamical equations for the two-time correlation functions will involve the bare coupling constant $b$. This one will be understood as a function of the cutoff $\Lambda$. We will solve the dynamical equations for a finite value of the cutoff. In the static case, we will show that the final formula obtained for the two-time correlation functions can be written with the renormalized parameters.

At finite temperature, we can isolate the divergent part of $G_{0}(\mathbf{x}, \mathbf{x})$ according to :

$$
G_{0}(\mathbf{x}, \mathbf{x})=\int \frac{d^{3} k}{(2 \pi)^{3}} \frac{1}{2 \omega_{k}}+\int \frac{d^{3} k}{(2 \pi)^{3}} \frac{1}{\omega_{k}} \frac{1}{e^{\beta \omega_{k}}-1}
$$

Therefore, the gap equation at finite temperature writes :

$$
\begin{aligned}
m^{2}(\beta)= & \epsilon \mu^{2}+\frac{g_{R}(\mu)}{16 \pi^{2}} m^{2}(\beta) \log \left(\frac{m^{2}(\beta)}{\mu^{2}}\right) \\
& +\frac{g_{R}(\mu)}{16 \pi^{2}} m^{2}(\beta) \int \frac{d^{3} k}{(2 \pi)^{3}} \frac{1}{\omega_{k}} \frac{1}{e^{\beta \omega_{k}}-1}
\end{aligned}
$$

\subsubsection{Two-time correlation functions :}

The first derivatives of $W\left(J, t_{0}\right)=-i \ln \mathcal{Z}_{s t}$ with respect to the sources are equal to the expectations values with the index $c$. Indeed, the functional $\mathcal{Z}$ depend on the sources both explicitly and implicitly since the approximate solutions for $\mathcal{D}(t)$ and $\mathcal{A}(t)$ depend 
on the sources. However, at the stationary point, only the explicit dependence contributes to the first derivative :

$$
\frac{d \mathcal{Z}}{d J_{j}(t)}=\frac{\delta \mathcal{Z}}{\delta J_{j}(t)}=i \operatorname{Tr}\left(\mathcal{D}(t) \mathcal{A}(t) Q_{j}\right)
$$

which gives for instance :

$$
\frac{\delta W}{\delta J_{\Phi}(\mathbf{x}, t)}=\varphi_{c}(\mathbf{x}, t)
$$

The expressions for the second derivatives of $W$ are much more complicated. The introduction of sources coupled to the composite operators $\Phi(\mathbf{x}) \Phi(\mathbf{y}), \Phi(\mathbf{x}) \Pi(\mathbf{y})$ and $\Pi(\mathbf{x}) \Pi(\mathbf{y})$ together with eq. (2.68) allows us to obtain dynamical equations for the two-time correlation functions with three or four field operators merely from the expansion of the expectation values $\alpha_{c}$ and $\Xi_{c}$ at the first order in powers of the sources.

From the first order corrections $\alpha_{c}-\alpha^{(0)}$ and $\Xi_{c}-\Xi^{(0)}$, we define the two-time correlation functions $\beta$ and $\Sigma$ ( $\beta$ is a vector and $\Sigma$ is a matrix) :

$$
\begin{aligned}
\alpha_{c}(\mathbf{x}, t)- & \alpha^{(0)}(\mathbf{x}, t) \simeq i \int_{t_{0}}^{\infty} d t^{\prime \prime}\left\{\int d^{3} y \beta^{\Phi}\left(\mathbf{x}, \mathbf{y}, t, t^{\prime \prime}\right) J^{\Phi}\left(\mathbf{y}, t^{\prime \prime}\right)+\beta^{\Pi} J^{\Pi}\right. \\
& \left.+\int d^{3} x_{1} d^{3} x_{2} \beta^{\Phi \Phi}\left(\mathbf{x}, \mathbf{x}_{1}, \mathbf{x}_{2}, t, t^{\prime \prime}\right) J^{\Phi \Phi}\left(\mathbf{x}_{1}, \mathbf{x}_{2}, t^{\prime \prime}\right)+\beta^{\Phi \Pi} J^{\Phi \Pi}+\beta^{\Pi \Pi} J^{\Pi \Pi}\right\} \\
\Xi_{c}(\mathbf{x}, \mathbf{y}, t)- & \Xi^{(0)}(\mathbf{x}, \mathbf{y}, t) \simeq i \int_{t_{0}}^{+\infty} d t^{\prime \prime}\left\{\int d^{3} x_{1} \Sigma^{\Phi}\left(\mathbf{x}, \mathbf{y}, \mathbf{x}_{1}, t, t^{\prime \prime}\right) J^{\Phi}\left(\mathbf{x}_{1}, t^{\prime \prime}\right)+\Sigma^{\Pi} J^{\Pi}\right. \\
& \left.+\int d^{3} x_{1} d^{3} x_{2} \Sigma^{\Phi \Phi}\left(\mathbf{x}, \mathbf{y}, \mathbf{x}_{1}, \mathbf{x}_{2}, t, t^{\prime \prime}\right) J^{\Phi \Phi}\left(\mathbf{x}_{1}, \mathbf{x}_{2}, t^{\prime \prime}\right)+\Sigma^{\Phi \Pi} J^{\Phi \Pi}+\Sigma^{\Pi \Pi} J^{\Pi \Pi}\right\}
\end{aligned}
$$

We have :

$$
\beta_{1}^{\Phi \Phi}\left(\mathbf{x}, \mathbf{y}, \mathbf{z}, t, t^{\prime \prime}\right)=\frac{1}{2} \Sigma_{11}^{\Phi}\left(\mathbf{x}, \mathbf{y}, \mathbf{z}, t, t^{\prime \prime}\right) .
$$

The functions $\beta$ and $\Sigma$ provide approximations for the exact two-time correlation functions $C^{2}, C^{3}$ and $C^{4}$ defined by Eqs. (2.10)-(2.13) :

$$
\begin{aligned}
& C_{\Phi \Phi}^{2}\left(\mathbf{x}, \mathbf{y}, t, t^{\prime \prime}\right) \simeq \beta_{1}^{\Phi}\left(\mathbf{x}, \mathbf{y}, t, t^{\prime \prime}\right), \\
& C^{3}\left(\mathbf{x}, \mathbf{y}, \mathbf{z}, t, t^{\prime \prime}\right) \simeq \beta_{1}^{\Phi \Phi}\left(\mathbf{x}, \mathbf{y}, \mathbf{z}, t, t^{\prime \prime}\right) \\
& -\varphi^{(0)}\left(\mathbf{y}, t^{\prime \prime}\right)\left(\beta_{1}^{\Phi}\left(\mathbf{x}, \mathbf{z}, t, t^{\prime \prime}\right)+\varphi^{(0)}(\mathbf{x}, t) \varphi^{(0)}\left(\mathbf{z}, t^{\prime \prime}\right)\right), \\
& -\varphi^{(0)}\left(\mathbf{z}, t^{\prime \prime}\right)\left(\beta_{1}^{\Phi}\left(\mathbf{x}, \mathbf{y}, t, t^{\prime \prime}\right)+\varphi^{(0)}(\mathbf{x}, t) \varphi^{(0)}\left(\mathbf{y}, t^{\prime \prime}\right)\right) \\
& C^{4}\left(\mathbf{x}, \mathbf{y}, \mathbf{z}, \mathbf{u}, t, t^{\prime \prime}\right) \simeq \frac{1}{2} \Sigma_{11}^{\Phi \Phi}\left(\mathbf{x}, \mathbf{y}, \mathbf{z}, \mathbf{u}, t, t^{\prime \prime}\right) \\
& -\left(\beta_{1}^{\Phi}\left(\mathbf{x}, \mathbf{z}, t, t^{\prime \prime}\right)+\varphi^{(0)}(\mathbf{x}, t) \varphi^{(0)}\left(\mathbf{z}, t^{\prime \prime}\right)\right)\left(\beta_{1}^{\Phi}\left(\mathbf{y}, \mathbf{u}, t, t^{\prime \prime}\right)+\varphi^{(0)}(\mathbf{y}, t) \varphi^{(0)}\left(\mathbf{u}, t^{\prime \prime}\right)\right) \text {. } \\
& -\left(\beta_{1}^{\Phi}\left(\mathbf{x}, \mathbf{u}, t, t^{\prime \prime}\right)+\varphi^{(0)}(\vec{x}, t) \varphi^{(0)}\left(\mathbf{u}, t^{\prime \prime}\right)\right)\left(\beta_{1}^{\Phi}\left(\mathbf{y}, \mathbf{z}, t, t^{\prime \prime}\right)+\varphi^{(0)}(\mathbf{y}, t) \varphi^{(0)}\left(\mathbf{z}, t^{\prime \prime}\right)\right)
\end{aligned}
$$

The expansion in powers of the sources of the dynamical equations (2.45) and (2.47) for $\alpha_{c}$ and $\Xi_{c}$ yields :

$$
i \delta \dot{\alpha}_{c}=\tau\left(\delta w_{c}-\delta w_{K}^{c}\right)
$$




$$
\begin{aligned}
i \delta \dot{\Xi}_{c}=2 & {\left[\delta \Xi_{c} \mathcal{H}^{(0)} \tau-\tau \mathcal{H}^{(0)} \delta \Xi_{c}\right.} \\
& \left.+\Xi^{(0)}\left(\delta \mathcal{H}_{c}-\delta \mathcal{I}_{K}^{c}\right) \tau-\tau\left(\delta \mathcal{H}_{c}-\delta \mathcal{I}_{K}^{c}\right) \Xi^{(0)}\right] .
\end{aligned}
$$

In these equations the matrix $\mathcal{H}$ has to be evaluated for the TDHB solutions $\alpha^{(0)}, \Xi^{(0)}$ of eqs. (2.52) and (2.53). The variations of $w_{K}^{c}(\mathbf{x}, t)$ (eqs. (D.18)-(D.19) ) and of $\mathcal{I}_{K}^{c}(\mathbf{x}, \mathbf{y}, t)$ (eqs. (D.20)-(D.22) ) with respect to $J(t ")$ will give terms proportional to $\delta(t-t$ "). From the variations $\delta w^{c}$ and $\delta \mathcal{H}^{c}$, we define the matrices $t_{i j}, T_{i, j k}, r_{i j, k}, R_{i j, k l}$ (which are the analogues of the random phase approximation (RPA) kernel of Balian and Vénéroni [6]) :

$$
\begin{gathered}
\delta w_{i}^{c}(\mathbf{x}, t)=\int d^{3} y t_{i j}(\mathbf{x}, \mathbf{y}, t) \delta \alpha_{j}^{c}(\mathbf{y}, t)-\frac{1}{2} \int d^{3} y d^{3} z T_{i, j k}(\mathbf{x}, \mathbf{y}, \mathbf{z}, t) \delta \Xi_{k j}^{c}(\mathbf{z}, \mathbf{y}, t), \quad(2.77) \\
\delta \mathcal{H}_{i j}^{c}(\mathbf{x}, \mathbf{y}, t)=\int d^{3} z r_{i j, k}(\mathbf{x}, \mathbf{y}, \mathbf{z}, t) \delta \alpha_{k}^{c}(\mathbf{z}, t)-\frac{1}{2} \int d^{3} z d^{3} u R_{i j, k l}(\mathbf{x}, \mathbf{y}, \mathbf{z}, \mathbf{u}, t) \delta \Xi_{l k}^{c}(\mathbf{u}, \mathbf{z}, t) .
\end{gathered}
$$

These matrices depend on the TDHB solutions $\alpha^{(0)}$ and $\Xi^{(0)}$. Their expressions in the case of the $\Phi^{4}$ theory are given in Appendix D. With these notations, the dynamical equations for the two-time and two-point correlation function $\beta^{\Phi}$ and the two-time and three-point correlation function $\Sigma^{\Phi}$ write :

$$
\begin{aligned}
i \frac{d}{d t} \beta^{\Phi}\left(\mathbf{x}, \mathbf{y}, t, t^{\prime \prime}\right)=\tau \quad & {\left[t(\mathbf{x}, \mathbf{z}, t) \beta^{\Phi}\left(\mathbf{z}, \mathbf{y}, t, t^{\prime \prime}\right)-\frac{1}{2} T_{,(j k}(\mathbf{x}, \mathbf{z}, \mathbf{u}, t) \Sigma_{k j)}^{\Phi}\left(\mathbf{u}, \mathbf{z}, \mathbf{y}, t, t^{\prime \prime}\right)\right.} \\
\left.+i\left(\begin{array}{l}
1 \\
0
\end{array}\right) \delta(\mathbf{x}-\mathbf{y}) \delta\left(t-t^{\prime \prime}\right)\right] & \\
i \frac{d}{d t} \Sigma_{i j}^{\Phi}\left(\mathbf{x}, \mathbf{y}, \mathbf{x}_{1}, t, t^{\prime \prime}\right)= & 2\left[\Sigma^{\Phi} \mathcal{H}^{(0)} \tau-\tau \mathcal{H}^{(0)} \Sigma^{\Phi}\right. \\
& +\Xi^{(0)} r_{,(k} \beta_{k)}^{\Phi} \tau-\tau r_{,(k} \beta_{k)}^{\Phi} \Xi^{(0)} \\
& \left.-\frac{1}{2} \Xi^{(0)} R_{,(k l} \Sigma_{l k)}^{\Phi} \tau+\frac{1}{2} \tau R_{,(k l} \Sigma_{l k)}^{\Phi} \Xi^{(0)}\right]_{i j}
\end{aligned}
$$

We obtain similar equations for the three-point functions $\beta^{\Phi \Phi}, \beta^{\Phi \Pi}, \beta^{\Pi \Pi}$ and the four-point functions $\Sigma^{\Phi \Phi}, \Sigma^{\Phi \Pi}$ and $\Sigma^{\Pi \Pi}$.

These dynamical equations are not sufficient since the boundary conditions are $\Xi_{a}^{-1}(t=+\infty)=0, \frac{1}{\Xi_{a}} \alpha_{a}(t=+\infty)=0$ and $\alpha_{d}\left(\mathbf{x}, t_{0}\right)=\alpha_{0}(\mathbf{x}), \Xi_{d}\left(\mathbf{x}, \mathbf{y}, t_{0}\right)=\Xi_{0}(\mathbf{x}, \mathbf{y})$. But, at this step, we have used the expansion of only one half of our variational parameters. We will use also the dynamical equations for the two-time functions $m$ and $l$ defined from the expansion in powers of the sources of $\Xi_{a}^{-1} \alpha_{a}$ and of $\alpha_{a}$ at the first order :

$$
\begin{gathered}
\frac{1}{\Xi_{a}} \alpha_{a}(t) \simeq i \int_{t_{0}}^{+\infty} d t^{\prime \prime} m^{k}\left(t^{\prime \prime}, t\right) J_{k}\left(t^{\prime \prime}\right), \\
\Xi_{a}^{-1}(t) \simeq i \int_{t_{0}}^{+\infty} d t^{\prime \prime} l^{k}\left(t^{\prime \prime}, t\right) J_{k}\left(t^{\prime \prime}\right) .
\end{gathered}
$$

(We have ommitted the space indices) 
From the relations of Appendix C, we will be abble to obtain relations between the functions $\Sigma^{k}\left(t_{0}, t^{\prime \prime}\right)$ and $l^{k}\left(t^{\prime \prime}, t_{0}\right)$ one the one hand and the functions $\beta^{k}\left(t_{0}, t^{\prime \prime}\right)$ and $m^{k}\left(t^{\prime \prime}, t_{0}\right)-l^{k}\left(t^{\prime \prime}, t_{0}\right) \alpha_{0}$ on the other hand. Indeed, that at the first order :

$$
\delta \Xi_{a}^{-1}=-\frac{1}{\Xi_{a}} \delta \Xi_{a} \frac{1}{\Xi_{a}} \simeq-\frac{1}{\Xi_{a}+\Xi_{d}} \delta \Xi_{a} \frac{1}{\Xi_{a}+\Xi_{d}},
$$

From eqs. (C.5) and (C.2), we obtain at the first order :

$$
\delta \Xi_{c}=-\left(\Xi^{(0)}-\tau\right) \delta \Xi_{a}^{-1}\left(\Xi^{(0)}+\tau\right)+\delta \Xi_{d}
$$

and

$$
\delta \alpha_{c}=\left(\Xi^{(0)}-\tau\right) \delta\left(\frac{\alpha_{a}}{\Xi_{a}+\Xi_{d}}\right)+\left(\Xi^{(0)}-\tau\right) \frac{1}{\Xi_{a}+\Xi_{d}} \delta \Xi_{a} \frac{1}{\Xi_{a}+\Xi_{d}} \alpha^{(0)}+\delta \alpha_{d} .
$$

At the initial time, $\delta \Xi_{d}=0, \delta \alpha_{d}=0$ and we have the following relations between the two-time functions :

$$
\begin{gathered}
\Sigma^{k}\left(t_{0}, t^{\prime \prime}\right)=-\left(\Xi_{0}-\tau\right) l^{k}\left(t^{\prime \prime}, t_{0}\right)\left(\Xi_{0}+\tau\right) \\
\beta^{k}\left(t_{0}, t^{\prime \prime}\right)=\left(\Xi_{0}-\tau\right)\left(m^{k}\left(t^{\prime \prime}, t_{0}\right)-l^{k}\left(t^{\prime \prime}, t_{0}\right) \alpha_{0}\right)
\end{gathered}
$$

By using the dynamical equation for $\Xi_{a}^{-1}$ at the first order

$$
i \frac{d}{d t}\left(\Xi_{a}^{-1}\right)=-\mathcal{H}_{c}+\mathcal{H}_{b}+\mathcal{I}_{K}^{c}+2 \Xi_{a}^{-1} \tau \mathcal{H}^{(0)}-2 \mathcal{H}^{(0)} \tau \Xi_{a}^{-1}
$$

and the variations $\delta w_{c}-\delta w_{b}$ and $\delta \mathcal{H}_{c}-\delta \mathcal{H}_{b}$, we obtain a backward dynamical equation for $l^{k}\left(t^{\prime \prime}, t\right)$ :

$$
\begin{aligned}
i \frac{d}{d t} l^{k}\left(t^{\prime \prime}, t\right)_{i j}= & 2 r_{i j, k} \tau_{k l}\left(m^{k}-l^{k} \alpha^{(0)}\right)_{l}-R_{i j, k l}\left(\Xi^{(0)} l \tau-\tau l \Xi^{(0)}\right)_{l k} \\
& +\left[2 l^{k} \tau \mathcal{H}^{(0)}-2 \mathcal{H}^{(0)} \tau l^{k}-i \frac{\delta \mathcal{I}_{K}^{c}}{\delta J_{k}} \delta\left(t-t^{\prime \prime}\right)\right]_{i j}
\end{aligned}
$$

where the matrices $r$ and $R$ have been defined in (2.78).

Similarly, from the dynamical equation for $\Xi_{a}^{-1} \alpha_{a}$ at the first order, we obtain a backward dynamical equation for $m^{k}\left(t^{\prime \prime}, t\right)$. It is actually more convenient to consider the function $m^{k}\left(t^{\prime \prime}, t\right)-l^{k}\left(t^{\prime \prime}, t\right) \alpha^{(0)}(t)$ which is solution of :

$$
\begin{aligned}
i \frac{d}{d t}\left(m^{k}\left(t^{\prime \prime}, t\right)-l^{k}\left(t^{\prime \prime}, t\right) \alpha^{(0)}(t)\right)= & t \tau\left(m^{k}\left(t^{\prime \prime}, t\right)-l^{k}\left(t^{\prime \prime}, t\right) \alpha^{(0)}(t)\right) \\
& -\frac{1}{2} T_{, j k}\left(\Xi^{(0)} l^{k} \tau-\tau l^{k} \Xi^{(0)}\right)_{k j}+\frac{i}{2} \frac{\delta w_{K}^{c}}{\delta J_{k}} \delta\left(t-t^{\prime \prime}\right)
\end{aligned},
$$

where the matrices $t$ and $T$ have been defined in (2.77). The reason to use this function will appear with the introduction of the function $F\left(t^{\prime}, t^{\prime \prime}\right)$ bellow (see 2.100). 
The boundary conditions for these two equations are :

$$
\begin{gathered}
l\left(t^{\prime \prime}, t\right)=0, m\left(t^{\prime \prime}, t\right)=0 \quad \text { pour } t>t^{\prime \prime} \\
l^{\Phi}\left(t^{\prime \prime}, t^{\prime \prime}\right)=0, l^{\Pi}\left(t^{\prime \prime}, t^{\prime \prime}\right)=0 \\
l_{11}^{\Phi \Phi}\left(\mathbf{x}, \mathbf{y}, \mathbf{z}, \mathbf{u}, t^{\prime \prime}, t^{\prime \prime}\right)=-\frac{1}{2}\left(\delta^{3}(\mathbf{x}-\mathbf{z}) \delta^{3}(\mathbf{y}-\mathbf{u})+\delta^{3}(\mathbf{x}-\mathbf{u}) \delta^{3}(\mathbf{y}-\mathbf{z})\right) \\
l_{12}^{\Phi \Pi}\left(t^{\prime \prime}, t^{\prime \prime}\right)=2 i \quad, \quad l_{21}^{\Phi \Pi}\left(t^{\prime \prime}, t^{\prime \prime}\right)=2 i \quad, \quad l_{22}^{\Pi \Pi}\left(t^{\prime \prime}, t^{\prime \prime}\right)=1
\end{gathered}
$$

The other elements of the matrix $l$ are equal to zero.

$$
\begin{gathered}
m_{1}^{\Phi}\left(\mathbf{x}, \mathbf{y}, t^{\prime \prime}, t^{\prime \prime}\right)=-\frac{1}{2} \delta^{3}(\mathbf{x}-\mathbf{y}), m_{2}^{\Pi}\left(\mathbf{x}, \mathbf{y}, t^{\prime \prime}, t^{\prime \prime}\right)=-\frac{i}{2} \delta^{3}(\mathbf{x}-\mathbf{y}) \\
m_{1}^{\Phi \Phi}\left(\mathbf{x}, \mathbf{x}_{1}, \mathbf{x}_{2}, t^{\prime \prime}, t^{\prime \prime}\right)-l_{1 i}^{\Phi \Phi}\left(t^{\prime \prime}, t^{\prime \prime}\right) \alpha_{i}^{(0)}\left(t^{\prime \prime}\right)=-\varphi^{(0)}\left(\mathbf{x}_{2}, t^{\prime \prime}\right) \delta^{3}\left(\mathbf{x}-\mathbf{x}_{1}\right)+\text { sym. } \\
m_{1}^{\Phi \Pi}\left(\mathbf{x}, \mathbf{x}_{1}, \mathbf{x}_{2}, t^{\prime \prime}, t^{\prime \prime}\right)-l_{1 i}^{\Phi \Pi}\left(t^{\prime \prime}, t^{\prime \prime}\right) \alpha_{i}^{(0)}\left(t^{\prime \prime}\right)=-\pi^{(0)}\left(\mathbf{x}_{2}, t^{\prime \prime}\right) \delta^{3}\left(\mathbf{x}-\mathbf{x}_{1}\right)+\text { sym. } \\
m_{2}^{\Phi \Pi}\left(\mathbf{x}, \mathbf{x}_{1}, \mathbf{x}_{2}, t^{\prime \prime}, t^{\prime \prime}\right)-l_{2 i}^{\Phi \Pi}\left(t^{\prime \prime}, t^{\prime \prime}\right) \alpha_{i}^{(0)}\left(t^{\prime \prime}\right)=-i \varphi^{(0)}\left(\mathbf{x}_{2}, t^{\prime \prime}\right) \delta^{3}\left(\mathbf{x}-\mathbf{x}_{\mathbf{1}}\right)+\text { sym } \\
m_{2}^{\Pi \Pi}\left(\mathbf{x}, \mathbf{x}_{1}, \mathbf{x}_{2}, t^{\prime \prime}, t^{\prime \prime}\right)-l_{2 i}^{\Pi \Pi}\left(t^{\prime \prime}, t^{\prime \prime}\right) \alpha_{i}^{(0)}\left(t^{\prime \prime}\right)=-i \pi^{(0)}\left(\mathbf{x}_{2}, t^{\prime \prime}\right) \delta^{3}\left(\mathbf{x}-\mathbf{x}_{\mathbf{1}}\right)+\text { sym }
\end{gathered}
$$

where sym. means the same terms obtained by exchanging $x_{2}, x$ and $x_{1}$.

The same matrices $t, T, r, R$ coming from the expansion of $\langle H>$ at the second order arround the TDHB solution appear in equations (2.79),(2.80) for $\beta$ and $\Sigma$ and in equations (2.89), 2.90) for $m$ and $l$. This leads to consider the following quantity :

$$
F^{k}\left(t^{\prime}, t^{\prime \prime}\right)=2\left(\tilde{m}^{k}\left(t^{\prime}, t\right)-\tilde{\alpha}^{(0)}(t) l^{k}\left(t^{\prime}, t\right)\right) \beta^{k}\left(t, t^{\prime \prime}\right)-\frac{1}{2} \operatorname{tr}\left(l^{k}\left(t^{\prime}, t\right) \Sigma^{k}\left(t, t^{\prime \prime}\right)\right)
$$

where $\tilde{m}$ is the transpozed vector of $m$. More explicitly, by reintroducing the space coordinates, $F^{\Phi}\left(t^{\prime}, t^{\prime \prime}\right)$ writes in the case of the functions $m^{\Phi}, l^{\Phi}, \beta^{\Phi}$ and $\Sigma^{\Phi}$ :

$$
\begin{aligned}
F^{\Phi}\left(t^{\prime}, t^{\prime \prime}\right)= & 2 \int d^{3} x\left(\tilde{m}^{\Phi}\left(\mathbf{x}, \mathbf{x}_{1}, t^{\prime}, t\right)-\int d^{3} u \tilde{\alpha}^{(0)}(\mathbf{u}, t) l^{\Phi}\left(\mathbf{u}, \mathbf{x}, \mathbf{x}_{1}, t^{\prime}, t\right)\right) \beta^{\Phi}\left(\mathbf{x}_{1}, \mathbf{x}_{2}, t, t^{\prime \prime}\right) \\
& -\frac{1}{2} \int d^{3} x d^{3} z \operatorname{tr}\left(l^{\Phi}\left(\mathbf{x}, \mathbf{z}, \mathbf{x}_{1}, t^{\prime}, t\right) \Sigma^{\Phi}\left(\mathbf{z}, \mathbf{x}, \mathbf{x}_{2}, t, t^{\prime \prime}\right)\right)
\end{aligned}
$$

The quantity $F^{k}$ is independent of the time $t$ : from the dynamical equations (2.79), (2.80) and $(2.89),(2.90)$, one shows that its derivative with respect to $t$ is equal to a sum of functions $\delta\left(t-t^{\prime}\right)$ and $\delta\left(t-t^{\prime \prime}\right)$ :

$$
\begin{aligned}
i \frac{d}{d t} F^{k}= & i \tilde{\beta}^{k}\left(t, t^{\prime \prime}\right) \frac{\delta w_{K}^{c}}{\delta J_{k}} \delta\left(t-t^{\prime}\right)+i 2\left(\tilde{m}^{k}\left(t^{\prime}, t\right)-\tilde{\alpha}^{(0)}(t) l^{k}\left(t^{\prime}, t\right)\right) \tau \frac{\delta w^{c}}{\delta J_{k}} \delta\left(t-t^{\prime \prime}\right) \\
& -\frac{1}{2} \operatorname{tr}\left\{i \frac{\delta \mathcal{I}_{\mathcal{K}}}{\delta J_{k}} \Sigma\left(t, t^{\prime \prime}\right) \delta\left(t-t^{\prime}\right)+i l^{k}\left(t^{\prime}, t\right) \Xi^{(0)}(t) \frac{\delta \mathcal{I}_{K}}{\delta J_{k}} \tau \delta\left(t-t^{\prime \prime}\right)-i l^{k}\left(t^{\prime}, t\right) \tau \frac{\delta \mathcal{I}_{K}}{\delta J_{k}} \Xi^{(0)}(t) \delta\left(t-t^{\prime \prime}\right)\right\}
\end{aligned}
$$


The fact that $F^{k}$ does not depend on the time $t$ will save us to solve one dynamical equation.

By considering the local sources $J^{\Phi}(\mathbf{x}, t)$ and by integrating equation (2.102) from $t=+\infty$ to $t=t_{0}$, we obtain an approximation for the two-time and two-point function. For $t^{\prime \prime}>t^{\prime}$ :

$$
\begin{aligned}
\beta_{1}^{\Phi}\left(t^{\prime}, t^{\prime \prime}\right)= & 2\left[\tilde{m}^{\Phi}\left(t^{\prime}, t_{0}\right)-\tilde{\alpha}_{0} l^{\Phi}\left(t^{\prime}, t_{0}\right)\right] \beta^{\Phi}\left(t_{0}, t^{\prime \prime}\right) \\
& -\frac{1}{2} \operatorname{tr}\left[l^{\Phi}\left(t^{\prime}, t_{0}\right) \Sigma^{\Phi}\left(t_{0}, t^{\prime \prime}\right)\right]
\end{aligned} .
$$

In the uniform case, we have in momentum space :

$$
\begin{aligned}
\beta_{1}^{\Phi}\left(\mathbf{p}, t^{\prime}, t^{\prime \prime}\right)= & 2\left(\tilde{m}^{\Phi}\left(-\mathbf{p}, t^{\prime}, t_{0}\right)-\tilde{\alpha}_{0} l^{\Phi}\left(0, \mathbf{p},-\mathbf{p}, t^{\prime}, t_{0}\right)\right) \beta^{\Phi}\left(\mathbf{p}, t_{0}, t^{\prime \prime}\right) \\
& -\frac{1}{2} \int d^{3} k \operatorname{tr}\left[l^{\Phi}\left(\mathbf{p}+\mathbf{k},-\mathbf{k},-\mathbf{p}, t^{\prime}, t_{0}\right) \Sigma^{\Phi}\left(\mathbf{k},-\mathbf{p}-\mathbf{k}, \mathbf{p}, t_{0}, t^{\prime \prime}\right)\right]
\end{aligned}
$$

Our conventions for the Fourrier transforms are defined in Appendix D.

These two last expressions will still be valid when we will optimize with respect to the initial state in section 3. On the contrary, the following expression will not be valid in section 3. By using (2.86) and (2.87), we obtain :

$$
\begin{aligned}
\beta_{1}^{\Phi}\left(t^{\prime}, t^{\prime \prime}\right)= & 2\left(\tilde{m}^{\Phi}\left(t^{\prime}, t_{0}\right)-\tilde{\alpha}_{0} l^{\Phi}\left(t^{\prime}, t_{0}\right)\right)\left(\Xi_{0}-\tau\right)\left(m^{\Phi}\left(t^{\prime \prime}, t_{0}\right)-l^{\Phi}\left(t^{\prime \prime}, t_{0}\right) \alpha_{0}\right) \\
& +\frac{1}{2} \operatorname{tr}\left[l^{\Phi}\left(t^{\prime}, t_{0}\right)\left(\Xi_{0}-\tau\right) l^{\Phi}\left(t^{\prime \prime}, t_{0}\right)\left(\Xi_{0}+\tau\right)\right]
\end{aligned}
$$

For $t^{\prime}>t^{\prime \prime}$, one interchanges the operators $\phi^{H}\left(t^{\prime}, t_{0}\right)$ and $\phi^{H}\left(t^{\prime \prime}, t_{0}\right)$. By using the expression of the retarded Green functions (see bellow), we show :

$$
\begin{aligned}
\beta_{1}^{\Phi}\left(t^{\prime}, t^{\prime \prime}\right)= & 2\left(\tilde{m}^{\Phi}\left(t^{\prime \prime}, t_{0}\right)-\tilde{\alpha}_{0} l^{\Phi}\left(t^{\prime \prime}, t_{0}\right)\right)\left(\Xi_{0}-\tau\right)\left(m^{\Phi}\left(t^{\prime}, t_{0}\right)-l^{\Phi}\left(t^{\prime}, t_{0}\right) \alpha_{0}\right) \\
& +\frac{1}{2} \operatorname{tr}\left[l^{\Phi}\left(t^{\prime \prime}, t_{0}\right)\left(\Xi_{0}-\tau\right) l^{\Phi}\left(t^{\prime}, t_{0}\right)\left(\Xi_{0}+\tau\right)\right]
\end{aligned}
$$

For the correlation functions with the anti-T-product, it is sufficient to replace in the functional $\mathcal{Z}(2.36)$ the source term $\mathcal{K}_{c}$ by the analogous term $\mathcal{K}_{b}$. This is equivalent to exchange the matrices $\Xi_{0}-\tau$ and $\Xi_{0}+\tau$ in the previous formula (or to exchange $t^{\prime}$ and $\left.t^{\prime \prime}\right)$.

From the function $F^{k}\left(t^{\prime}, t^{\prime \prime}\right)$, we obtain also, by considering now the bilocal sources $J^{\Phi \Phi}(\mathbf{x}, \mathbf{y}, t)$, an expression which relates the approximations for the two-time and threepoint function $\beta_{1}^{\Phi \Phi}\left(t^{\prime}, t^{\prime \prime}\right)$ and for the two-time and four-point function $\Sigma_{11}^{\Phi \Phi}\left(t^{\prime}, t^{\prime \prime}\right)$. For $t^{\prime \prime}>t^{\prime}:$

$$
\begin{aligned}
& \beta_{1}^{\Phi \Phi}\left(\mathbf{x}_{1}, \mathbf{x}_{1}^{\prime}, \mathbf{x}_{2}^{\prime}, t^{\prime}, t^{\prime \prime}\right) \varphi^{(0)}\left(\mathbf{x}_{2}, t^{\prime}\right)+\beta_{1}^{\Phi \Phi}\left(\mathbf{x}_{2}, \mathbf{x}_{1}^{\prime}, \mathbf{x}_{2}^{\prime}, t^{\prime}, t^{\prime \prime}\right) \varphi^{(0)}\left(\mathbf{x}_{1}, t^{\prime}\right) \\
& -\frac{1}{4}\left(\Sigma_{11}^{\Phi \Phi}\left(\mathbf{x}_{1}, \mathbf{x}_{2}, \mathbf{x}_{1}^{\prime}, \mathbf{x}_{2}^{\prime}, t^{\prime}, t^{\prime \prime}\right)+\Sigma_{11}^{\Phi}\left(\mathbf{x}_{2}, \mathbf{x}_{1}, \mathbf{x}_{1}^{\prime}, \mathbf{x}_{2}^{\prime}, t^{\prime}, t^{\prime \prime}\right)\right)= \\
& -2 \int d^{3} x\left(\tilde{m}^{\Phi \Phi}\left(\mathbf{x}, \mathbf{x}_{1}, \mathbf{x}_{2}, t^{\prime}, t_{0}\right)-\int d^{3} u \tilde{\alpha}_{0}(\mathbf{u}) l^{\Phi \Phi}\left(\mathbf{u}, \mathbf{x}, \mathbf{x}_{1}, \mathbf{x}_{2}, t^{\prime}, t_{0}\right)\right) \beta^{\Phi \Phi}\left(\mathbf{x}, \mathbf{x}_{1}^{\prime}, \mathbf{x}_{2}^{\prime}, t_{0}, t^{\prime \prime}\right) \\
& +\frac{1}{2} \int d^{3} x d^{3} z \operatorname{tr}\left[l^{\Phi \Phi}\left(\mathbf{x}, \mathbf{z}, \mathbf{x}_{1}, \mathbf{x}_{2}, t^{\prime}, t_{0}\right) \Sigma^{\Phi \Phi}\left(\mathbf{z}, \mathbf{x}, \mathbf{x}_{1}^{\prime}, \mathbf{x}_{2}^{\prime}, t_{0}, t^{\prime \prime}\right)\right]
\end{aligned} .
$$


In the uniform case, we have in momentum space :

$$
\begin{aligned}
& {\left[\beta_{1}^{\Phi \Phi}\left(\mathbf{p}_{1}, \mathbf{q},-\left(\mathbf{p}_{1}+\mathbf{q}\right), t^{\prime}, t^{\prime \prime}\right) \delta^{3}\left(\mathbf{p}_{2}\right)+\beta_{1}^{\Phi \Phi}\left(\mathbf{p}_{2}, \mathbf{q},-\left(\mathbf{p}_{2}+\mathbf{q}\right), t^{\prime}, t^{\prime \prime}\right) \delta^{3}\left(\mathbf{p}_{1}\right)\right] \varphi^{(0)}\left(t^{\prime}\right)} \\
& -\frac{1}{4}\left[\Sigma_{11}^{\Phi \Phi}\left(\mathbf{p}_{1}, \mathbf{p}_{2}, \mathbf{q},-\left(\mathbf{p}_{1}+\mathbf{p}_{2}+\mathbf{q}\right), t^{\prime}, t^{\prime \prime}\right)+\Sigma_{11}^{\Phi \Phi}\left(\mathbf{p}_{2}, \mathbf{p}_{1}, \mathbf{q},-\left(\mathbf{p}_{1}+\mathbf{p}_{2}+\mathbf{q}\right), t^{\prime}, t^{\prime \prime}\right)\right]= \\
& -2\left[\tilde{m}^{\Phi \Phi}\left(-\mathbf{p}_{1}-\mathbf{p}_{2}, \mathbf{p}_{1}, \mathbf{p}_{2}, t^{\prime}, t_{0}\right)-\tilde{\alpha}_{0} l^{\Phi \Phi}\left(0,-\mathbf{p}_{1}-\mathbf{p}_{2}, \mathbf{p}_{1}, \mathbf{p}_{2}, t^{\prime}, t_{0}\right)\right] \\
& \times \beta^{\Phi \Phi}\left(\mathbf{p}_{1}+\mathbf{p}_{2}, \mathbf{q},-\mathbf{q}-\mathbf{p}_{1}-\mathbf{p}_{2}, t_{0}, t^{\prime \prime}\right) \\
& +\frac{1}{2} \int d^{3} k \operatorname{tr}\left[l^{\Phi \Phi}\left(\mathbf{k}-\mathbf{p}_{1}-\mathbf{p}_{2},-\mathbf{k}, \mathbf{p}_{1}, \mathbf{p}_{2}, t^{\prime}, t_{0}\right) \Sigma^{\Phi \Phi}\left(\mathbf{k},-\mathbf{k}+\mathbf{p}_{1}+\mathbf{p} \mathbf{p}_{2}, \mathbf{q},-\mathbf{q}-\mathbf{p}_{1}-\mathbf{p}_{2}, t_{0}, t^{\prime \prime}\right)\right]
\end{aligned}
$$

Let us recall that the function $\Sigma_{11}^{\Phi}$ is related to $\beta_{1}^{\Phi \Phi}\left(t^{\prime}, t^{\prime \prime}\right)$ by : $\beta_{1}^{\Phi \Phi}=\frac{1}{2} \Sigma_{11}^{\Phi}$. By comparing the dynamical equations for $\beta_{i}^{\Phi \Phi}\left(t^{\prime}, t^{\prime \prime}\right)$ and $\Sigma_{i j}^{\Phi}\left(t^{\prime}, t^{\prime \prime}\right)$ we obtain on the other hand : $\beta_{2}^{\Phi \Phi}=\Sigma_{12}^{\Phi}+\Sigma_{21}^{\Phi}$ and an expression for $\Sigma_{22}^{\Phi}$ in terms of $\beta_{1}^{\Phi \Phi}$ and two integrales over the momenta which involve $\Sigma_{11}^{\Phi \Phi}$ and $\Sigma_{11}^{\Phi}=2 \beta_{1}^{\Phi \Phi}$. The functions $\Sigma_{i j}^{\Phi}$ are therefore completly known if $\beta_{1}^{\Phi \Phi}$ and $\Sigma_{11}^{\Phi \Phi}$ are known. However, we note a difficulty in the asymmetric phase since we have two equations (2.103) and (2.107) for the three functions $\beta_{1}^{\Phi}\left(t^{\prime}, t^{\prime \prime}\right), \beta_{1}^{\Phi \Phi}\left(t^{\prime}, t^{\prime \prime}\right)$ and $\Sigma_{11}^{\Phi \Phi}\left(t^{\prime}, t^{\prime \prime}\right)$.

In the symmetric phase, equations (2.105) and (2.107) simplify. Indeed, from the boundary condition $l^{\Phi}\left(t^{\prime}, t^{\prime}\right)=0$ and the dynamical equation (2.89) for $l$, we deduce $l^{\Phi}\left(t^{\prime}, t\right)=0$ for $t \leq t^{\prime}$. Similarly, $m^{\Phi \Phi}\left(t^{\prime}, t\right)-l^{\Phi \Phi}\left(t^{\prime}, t\right) \alpha^{(0)}(t)=0$ for $t \leq t^{\prime}$. Therefore, we have in the symmetric phase and for $t^{\prime \prime}>t^{\prime}$ :

$$
\beta_{1}^{\Phi}\left(\mathbf{p}, t^{\prime}, t^{\prime \prime}\right)=2 \tilde{m}^{\Phi}\left(\mathbf{p}, t^{\prime}, t_{0}\right)\left(\Xi_{0}(\mathbf{p})-\tau\right) m^{\Phi}\left(\mathbf{p}, t^{\prime \prime}, t_{0}\right)
$$

and for the four-point function :

$$
\begin{aligned}
\Sigma_{11}^{\Phi \Phi}\left(\mathbf{p}_{1}, \mathbf{p}_{2}, \mathbf{p}_{3},-\sum \mathbf{p}_{i}, t^{\prime}, t^{\prime \prime}\right)=-\int \frac{d^{3} p}{(2 \pi)^{3}} & \operatorname{tr}\left[l^{\Phi \Phi}\left(\mathbf{p}-\mathbf{p}_{1}-\mathbf{p}_{2},-\mathbf{p}, \mathbf{p}_{1}, \mathbf{p}_{2}, t^{\prime}, t_{0}\right)\right. \\
& \left.\Sigma_{11}^{\Phi \Phi}\left(\mathbf{p},-\mathbf{p}+\mathbf{p}_{1}+\mathbf{p}_{2}, \mathbf{p}_{3},-\sum \mathbf{p}_{i}, t_{0}, t^{\prime \prime}\right)\right]
\end{aligned}
$$

By using (2.86) :

$$
\begin{aligned}
\Sigma_{11}^{\Phi \Phi}\left(\mathbf{p}_{1}, \mathbf{p}_{2}, \mathbf{p}_{3},-\sum \mathbf{p}_{i}, t^{\prime}, t^{\prime \prime}\right)= & \int \frac{d^{3} p}{(2 \pi)^{3}} \operatorname{tr}\left[l^{\Phi \Phi}\left(\mathbf{p}-\mathbf{p}_{1}-\mathbf{p}_{2},-\mathbf{p}, \mathbf{p}_{1}, \mathbf{p}_{2}, t^{\prime}, t_{0}\right)\left(\Xi_{0}(\mathbf{p})-\tau\right)\right. \\
& \left.l^{\Phi \Phi}\left(\mathbf{p},-\mathbf{p}+\mathbf{p}_{1}+\mathbf{p}_{2}, \mathbf{p}_{3},-\sum \mathbf{p}_{i}, t^{\prime \prime}, t_{0}\right)\left(\Xi_{0}\left(-\mathbf{p}+\mathbf{p}_{1}+\mathbf{p}_{2}\right)+\tau\right)\right]
\end{aligned}
$$

Let us emphasize that it is $\Xi_{0}$, the static HB solution and not $\Xi^{(0)}$ which appears in expression (2.111).

For $t^{\prime}>t^{\prime \prime}$, we have, still in the symmetric phase :

$$
\begin{aligned}
\beta_{1}^{\Phi}\left(\mathbf{p}, t^{\prime}, t^{\prime \prime}\right) & =2 \tilde{m}^{\Phi}\left(\mathbf{p}, t^{\prime}, t_{0}\right)\left(\Xi_{0}(\mathbf{p})+\tau\right) m^{\Phi}\left(\mathbf{p}, t^{\prime \prime}, t_{0}\right) \\
\Sigma_{11}^{\Phi \Phi}\left(\mathbf{p}_{1}, \mathbf{p}_{2}, \mathbf{p}_{3},-\sum \mathbf{p}_{i}, t^{\prime}, t^{\prime \prime}\right)= & \int \frac{d^{3} p}{(2 \pi)^{3}} \operatorname{tr}\left[l^{\Phi \Phi}\left(\mathbf{p}-\mathbf{p}_{1}-\mathbf{p}_{2},-\mathbf{p}, \mathbf{p}_{1}, \mathbf{p}_{2}, t^{\prime \prime}, t_{0}\right)\left(\Xi_{0}(\mathbf{p})-\tau\right)\right. \\
& \left.l^{\Phi \Phi}\left(\mathbf{p},-\mathbf{p}+\mathbf{p}_{1}+\mathbf{p}_{2}, \mathbf{p}_{3},-\sum \mathbf{p}_{i}, t^{\prime}, t_{0}\right)\left(\Xi_{0}\left(-\mathbf{p}+\mathbf{p}_{1}+\mathbf{p}_{2}\right)+\tau\right)\right]
\end{aligned}
$$


To conclude, in order to obtain approximations for the causal correlation functions $C^{2}, C^{3}$ and $C^{4}$, we have first to solve the TDHB equations (2.52) and (2.53) for $\alpha^{(0)}(t)$ and $\Xi^{(0)}(t)$ and to evaluate the matrices $r, R, t$ and $T$ for the TDHB solutions. We then solve equations (2.89) and (2.90) for $l\left(t^{\prime \prime}, t\right)$ and $m\left(t^{\prime \prime}, t\right)-l\left(t^{\prime \prime}, t\right) \alpha^{(0)}(t)\left(t^{\prime \prime}>t \geq t_{0}\right)$ backward in time from $t^{\prime \prime}$ to $t_{0}$ with the boundary conditions (2.91)-(2.99). Finally, we evaluate expression (2.105). Therefore, it is not necessary to solve the dynamical equations (2.79) and 2.80$)$ for $\beta\left(t, t^{\prime \prime}\right)$ and $\Sigma\left(t, t^{\prime \prime}\right)$.

The expressions we obtain in this way for three-point and four point correlation functions are not completly satisfactory. Indeed, in the static case, when $\alpha^{(0)}(t)=\alpha_{0}$ and $\Xi^{(0)}(t)=\Xi_{0}$ are time-independent, the approximate correlation functions do not depend only on the time difference $t^{\prime}-t^{\prime \prime}$ as they should (the two-point function in the symmetric phase is a particular case). We will cure this artefact by optimizing with respect to the initial state in section 3 .

\section{Retarded Green Functions :}

The function $\beta_{1}^{\phi}$ is an approximation for the Green function with the time-ordered Green function :

$$
\begin{aligned}
C_{\Phi \Phi}^{2}\left(\mathbf{x}, \mathbf{y}, t, t^{\prime \prime}\right)= & \operatorname{Tr}\left(T \Phi^{H}\left(\mathbf{x}, t^{\prime}, t_{0}\right) \Phi^{H}\left(\mathbf{y}, t^{\prime \prime}, t_{0}\right) D\left(t_{0}\right)\right) \\
& -\operatorname{Tr}\left(\Phi^{H}\left(\mathbf{x}, t^{\prime}, t^{\prime \prime}\right) D\left(t_{0}\right)\right) \operatorname{Tr}\left(\Phi^{H}\left(\mathbf{y}, t^{\prime \prime}, t_{0}\right) D\left(t_{0}\right)\right)
\end{aligned} .
$$

To obtain the approximations for the anticausal two-time functions, it is sufficient to replace in the functional (2.36) the term $K_{c}$ by the analogous term $K_{b}$. Formula (2.105) and (2.106) are then interchanged.

The retarded Green function is defined by :

$$
\chi_{\Phi \Phi}\left(\mathbf{x}, \mathbf{y}, t^{\prime}, t^{\prime \prime}\right)=-i \Theta\left(t^{\prime}-t^{\prime \prime}\right) \operatorname{Tr}\left(\left[\Phi^{H}\left(\mathbf{x}, t^{\prime}, t_{0}\right), \Phi^{H}\left(\mathbf{y}, t^{\prime \prime}, t_{0}\right)\right] D\left(t_{0}\right)\right) .
$$

It is interesting to obtain an approximation for the retarded Green function. It is this Green function which appears in the study of the response of the system to a small external perturbation. Moreover, the KMS condition allows to relate the different components of the Green function in the real time formalism for Quantum Field Theories at finite temperature [17]. These components can all be expressed from the retarded Green function. The real parts of the time-ordered Green function $C^{2}$ and of the retarded Green function $\chi$ coincide. They describe phenomena such as Debye screening in a plasma in the problem of charged particules in an external electromagnetic field. But one has to consider the imaginary part of the retarded Green function to describe damping phenomena [18. The optimization with respect to the initial state in section 3 will allows to obtain approximated Green functions wich verify the KMS condition (the fluctuation-disspation theorem). 
From expression (2.106) and the analogous formula for the correlation function with the anti-T product, we obtain an approximation for the retarded Green function :

$$
\begin{aligned}
\chi_{\Phi \Phi}\left(\mathbf{x}, \mathbf{y}, t^{\prime}, t^{\prime \prime}\right)=-i \Theta\left(t^{\prime}-t^{\prime \prime}\right) & {\left[-4\left(\tilde{m}^{\Phi}\left(t^{\prime \prime}, t_{0}\right)-\tilde{\alpha}_{0} l^{\Phi}\left(t^{\prime \prime}, t_{0}\right)\right) \tau\left(m^{\Phi}\left(t^{\prime}, t_{0}\right)-l^{\Phi}\left(t^{\prime}, t_{0}\right) \alpha_{0}\right)\right.} \\
& \left.+\operatorname{tr}\left(l^{\Phi}\left(t^{\prime \prime}, t_{0}\right) \Xi_{0} l^{\Phi}\left(t^{\prime}, t_{0}\right) \tau\right)-\operatorname{tr}\left(l^{\Phi}\left(t^{\prime \prime}, t_{0}\right) \tau l^{\Phi}\left(t^{\prime}, t_{0}\right) \Xi_{0}\right)\right]
\end{aligned}
$$

In the symetric phase :

$$
\chi_{\Phi \Phi}\left(\vec{x}, \vec{y}, t^{\prime}, t^{\prime \prime}\right)=i \Theta\left(t^{\prime}-t^{\prime \prime}\right) 4 \tilde{m}^{\Phi}\left(t^{\prime \prime}, t_{0}\right) \tau m^{\Phi}\left(t^{\prime}, t_{0}\right) .
$$

The exact expression (2.115) remains identical if we translate the refence time $t_{0}$ to another time $t$ such as $t^{\prime \prime}>t>t_{0}$. By using the dynamical equation for $\Xi^{(0)}(t)$ and equations (2.89) and (2.90), we show that the approximation (2.116) has the same property. Indeed, if we call $R\left(t^{\prime}, t^{\prime \prime}\right)$ the expression obtained by replacing in the bracket of equation (2.116) $t_{0}$ by $t$ with $t_{0} \leq t<t^{\prime \prime}$, we have for $t^{\prime}>t^{\prime \prime}$ :

$$
\frac{d R}{d t}=2\left(\tilde{m}^{\Phi}\left(t^{\prime}, t\right)-\tilde{\alpha}^{(0)}(t)\right) l^{\Phi}\left(t^{\prime}, t\right) \tau\left(\begin{array}{l}
1 \\
0
\end{array}\right) \delta\left(t-t^{\prime \prime}\right)
$$

We deduce : $\frac{d R}{d t}=0$ pour $t<t^{\prime \prime}$. We thus have :

$$
\chi_{\Phi \Phi}\left(\mathbf{x}, \mathbf{y}, t^{\prime}, t^{\prime \prime}\right)=-i \Theta\left(t^{\prime}-t^{\prime \prime}\right) R\left(t^{\prime}, t^{\prime \prime}\right)
$$

When the time $t^{\prime}$ tends to $t^{\prime \prime}$, the exact formula (2.115) coincides with the commutator of two fields $\Phi^{H}\left(t^{\prime}, t_{0}\right)$ at equal time which is vanishing. The approximated expression gives also $\chi_{\Phi \Phi}\left(t^{\prime}, t^{\prime}\right)=0$ since $m_{1}^{\Phi}\left(t^{\prime \prime}, t^{\prime \prime}\right)=-\frac{1}{2}$ and $l^{\Phi}\left(t^{\prime \prime}, t^{\prime \prime}\right)=0$.

Similarly, we can obtain approximations for the retarded Green function with two field operators $\Phi \Phi$ defined according to :

$\chi_{\Phi \Phi, \Phi \Phi}\left(\mathbf{x}, \mathbf{y}, \mathbf{z}, \mathbf{u}, t^{\prime}, t^{\prime \prime}\right)=-i \theta\left(t^{\prime}-t^{\prime \prime}\right) \operatorname{Tr}\left(\left[\Phi^{H}\left(\mathbf{x}, t^{\prime}, t_{0}\right) \Phi^{H}\left(\mathbf{y}, t^{\prime}, t_{0}\right), \Phi^{H}\left(\mathbf{z}, t^{\prime \prime}, t_{0}\right) \Phi^{H}\left(\mathbf{u}, t^{\prime \prime}, t_{0}\right)\right] D\left(t_{0}\right)\right)$.

In the symmetric phase, from expression (2.113) and from the analogous formula for the correlation function with the anti-T product, we deduce an approximation for the retarded Green function with two field operators :

$$
\begin{aligned}
& \chi_{\Phi \Phi, \Phi \Phi}\left(\mathbf{p}_{1}, \mathbf{p}_{2}, \mathbf{p}_{3},-\sum \mathbf{p}_{i}, t^{\prime}, t^{\prime \prime}\right)=-i \theta\left(t^{\prime}-t^{\prime \prime}\right) \int \frac{d^{3} p}{(2 \pi)^{3}} \times \\
& \operatorname{tr}\left[l^{\Phi \Phi}\left(\mathbf{p}-\mathbf{p}_{1}-\mathbf{p}_{2},-\mathbf{p}, \mathbf{p}_{1}, \mathbf{p}_{2}, t^{\prime \prime}, t_{0}\right) \Xi_{0}(\mathbf{p}) l^{\Phi \Phi}\left(\mathbf{p},-\mathbf{p}+\mathbf{p}_{1}+\mathbf{p}_{2}, \mathbf{p}_{3},-\sum \mathbf{p}_{i}, t^{\prime}, t_{0}\right) \tau\right] \\
& -\operatorname{tr}\left[l^{\Phi \Phi}\left(\mathbf{p}-\mathbf{p}_{1}-\mathbf{p}_{2},-\mathbf{p}, \mathbf{p}_{1}, \mathbf{p}_{2}, t^{\prime \prime}, t_{0}\right) \tau l^{\Phi \Phi}\left(\mathbf{p},-\mathbf{p}+\mathbf{p}_{1}+\mathbf{p}_{2}, \mathbf{p}_{3},-\sum \mathbf{p}_{i}, t^{\prime}, t_{0}\right) \Xi_{0}\left(-\mathbf{p}+\mathbf{p}_{1}+\mathbf{p}_{2}\right)\right]
\end{aligned}
$$

Again, the right member of this equation does not depend on the time $t_{0}$. Indded, if we call $S\left(t^{\prime}, t^{\prime \prime}\right)$ the expression obtained by replacing in the right member $t_{0}$ by $t$, with 
$t_{0} \leq t<t^{\prime \prime}$, we have for $t^{\prime}>t^{\prime \prime}$ :

$$
\frac{d S}{d t}=-2 \operatorname{tr}\left[l^{\Phi \Phi}\left(t^{\prime}, t\right) \Xi_{0} \frac{\delta \mathcal{I}_{K}}{\delta J^{\Phi \Phi}} \tau-l^{\Phi \Phi}\left(t^{\prime}, t\right) \tau \frac{\delta \mathcal{I}_{K}}{\delta J^{\Phi \Phi}} \Xi_{0}\right] \delta\left(t-t^{\prime \prime}\right)
$$

and we deduce : $\frac{d S}{d t}=0$ for $t<t^{\prime \prime}$. We have therefore :

$$
\chi_{\Phi \Phi, \Phi \Phi}\left(\mathbf{p}_{1}, \mathbf{p}_{2}, \mathbf{p}_{3},-\sum \mathbf{p}_{i}, t^{\prime}, t^{\prime \prime}\right)=-i \theta\left(t^{\prime}-t^{\prime \prime}\right) S\left(t^{\prime}, t^{\prime \prime}\right)
$$

\section{The limit when the two times t' and t" coïncide :}

When $t^{\prime} \rightarrow t^{\prime \prime}, t^{\prime}=t^{\prime \prime}=t+0$, expression 2.105) for the approximated two point function becomes :

$$
\begin{aligned}
\beta_{1}^{\Phi}(t, t)= & 2\left(\tilde{m}^{\Phi}\left(t, t_{0}\right)-\tilde{\alpha}_{0} l^{\Phi}\left(t, t_{0}\right)\right)\left(\Xi_{0}-\tau\right)\left(m^{\Phi}\left(t, t_{0}\right)-l^{\Phi}\left(t, t_{0}\right) \alpha_{0}\right) \\
& +\frac{1}{2} \operatorname{tr}\left[l^{\Phi}\left(t, t_{0}\right)\left(\Xi_{0}-\tau\right) l^{\Phi}\left(t, t_{0}\right)\left(\Xi_{0}+\tau\right)\right]
\end{aligned}
$$

At the initial time $t=t_{0}$, we get as we should the TDHB result : $\beta_{1}^{\Phi}\left(t_{0}, t_{0}\right)=G_{0}$. Indeed, $m_{1}^{\Phi}\left(t_{0}, t_{0}\right)=-1 / 2$ and $l^{\Phi}\left(t_{0}, t_{0}\right)=0$. However for later times, this approximation is in general different from the result of the TDHB evolution : $\frac{1}{2} \Xi^{(0)}(t)=G^{(0)}(t)$. The case at equilibrium in the symmetric phase is a special case since, as we will show in section 3 , we have :

$$
\beta_{1}^{\Phi}(p, \Delta t=0)=G_{0}(p)
$$

This won't be true in the asymmetric phase. The result (2.125) is to relate to the fact that, at equilibrium in the symmetric phase, the two approximations for the two-time and two-point function obtained with or without the optimization with respect to the initial state coïncide (see below eq. (3.58)).

Let us examine the four-point function when the two times coïncide. In the symmetric phase, the limit $t^{\prime}=t^{\prime \prime}-0=t+0$ in expression (2.111) for the four-point function $\Sigma_{11}^{\phi \phi}$ gives :

$$
\begin{aligned}
\Sigma_{11}^{\Phi \Phi}\left(\mathbf{p}_{1}, \mathbf{p}_{2}, \mathbf{p}_{3},-\sum \mathbf{p}_{i}, t, t\right)= & \int \frac{d^{3} p}{(2 \pi)^{3}} \operatorname{tr}\left[l^{\Phi \Phi}\left(\mathbf{p}-\mathbf{p}_{1}-\mathbf{p}_{2},-\mathbf{p}, \mathbf{p}_{1}, \mathbf{p}_{2}, t, t_{0}\right)\left(\Xi_{0}(\mathbf{p})-\tau\right)\right. \\
& l^{\Phi \Phi}\left(\mathbf{p},-\mathbf{p}+\mathbf{p}_{1}+\mathbf{p}_{2}, \mathbf{p}_{3},-\sum \mathbf{p}_{i}, t_{0}, t\right)\left(\Xi_{0}\left(-\mathbf{p}+\mathbf{p}_{1}+\mathbf{p}_{2}\right)+\tau\right)
\end{aligned}
$$

The boundary conditions for the function $l^{\Phi \Phi}$ write

$$
l_{11}^{\Phi \Phi}\left(\mathbf{p}_{1}, \mathbf{p}_{2}, \mathbf{p}_{3},-\sum \mathbf{p}_{i}, t, t\right)=-\frac{1}{2}\left[\delta^{3}\left(\mathbf{p}_{1}+\mathbf{p}_{3}\right)+\delta^{3}\left(\mathbf{p}_{2}+\mathbf{p}_{3}\right)\right],
$$

the other matrix elements of $l^{\Phi \Phi}$ being equal to zero. We thus have at the initial time :

$$
\Sigma_{11}^{\Phi \Phi}\left(\mathbf{p}_{1}, \mathbf{p}_{2}, \mathbf{p}_{3},-\sum \mathbf{p}_{i}, t_{0}, t_{0}\right)=\frac{1}{2} \Xi_{11}^{0}\left(-\mathbf{p}_{1}\right) \Xi_{11}^{0}\left(\mathbf{p}_{2}\right)\left[\delta^{3}\left(\mathbf{p}_{1}+\mathbf{p}_{3}\right)+\delta^{3}\left(\mathbf{p}_{2}+\mathbf{p}_{3}\right)\right]
$$


The mean-field approximation for which $C_{4}=0$ corresponds to (see eq. (2.74)) :

$$
\begin{aligned}
\Sigma_{11}^{\phi \phi}(\mathbf{x}, \mathbf{y}, \mathbf{z}, \mathbf{u}, t, t) & =2 \beta_{1}^{\phi}(\mathbf{x}, \mathbf{z}, t, t) \beta_{1}^{\phi}(\mathbf{y}, \mathbf{u}, t, t)+2 \beta_{1}^{\phi}(\mathbf{x}, \mathbf{u}, t, t) \beta_{1}^{\phi}(\mathbf{y}, \mathbf{z}, t, t) \\
& =2 G^{(0)}(\mathbf{x}, \mathbf{z}, t) G^{(0)}(\mathbf{y}, \mathbf{u}, t)+2 G^{(0)}(\mathbf{x}, \mathbf{u}, t) G^{(0)}(\mathbf{y}, \mathbf{z}, t)
\end{aligned}
$$

In momentum space, this corresponds to :

$$
\Sigma_{11}^{\Phi \Phi}\left(\mathbf{p}_{1}, \mathbf{p}_{2}, \mathbf{p}_{3},-\sum \mathbf{p}_{i}, t, t\right)=2 \beta_{1}^{\Phi}\left(-\mathbf{p}_{1}, t, t\right) \beta_{1}^{\Phi}\left(\mathbf{p}_{2}, t\right)\left[\delta\left(\mathbf{p}_{3}+\mathbf{p}_{1}\right)+\delta\left(\mathbf{p}_{3}+\mathbf{p}_{2}\right)\right]
$$

At the initial time, we recover the result of the Wick theorem given by expression 2.128 in momentum space. However, for later times, owing to the correlations we have taken into account in the dynamics, approximation (2.126) for the four-point correlation function is different from the result of the Wick theorem.

In nonrelativistic many-body problems, the mean-field TDHF calculations correctly predict the expectation values but underestimate the fluctuations. Balian and Vénéroni have showed the advantage to use a variational principle adapted to calculate the fluctuations. For a pur state in the case of heavy-ions collisions, one obtains in this way a better agreement with experiments for the width of the mass distributions 19. The example of the quartic oscillator in one dimension shows also the usefullness of the method developped by Balian and Vénéroni to calculate the fluctuations of the operator $x^{2},\left\langle x^{4}>-<x^{2}\right\rangle^{2}$, though staying in a mean-field approach [14. 


\section{Optimization of the initial state}

\subsection{Introduction}

In physical situations, the initial state is not completly known. Only the expectation values of a few observables are known, for instance :

$$
\langle\Phi(\mathbf{x})\rangle_{t_{0}},\langle\Phi(\mathbf{x}) \Phi(\mathbf{y})\rangle_{t_{0}},\langle\Phi(\mathbf{x}) \Phi(\mathbf{y}) \Phi(\mathbf{z})\rangle_{t_{0}},\langle\Phi(\mathbf{x}) \Phi(\mathbf{y}) \Phi(\mathbf{z}) \Phi(\mathbf{u})\rangle_{t_{0}}
$$

The exact state $D\left(t_{0}\right)$ can be written as $\exp (-\beta \bar{H})$. The operator $\bar{H}$ is equal to the Hamiltonian of the system at thermal equilibrium but it can also contain other terms corresponding to conserved quantities, like the charge operator for a complex scalar field. For a nonequilibrium situation, the operator $\bar{H}$ is different from the Hamiltonian $H$.

We will approximate this initial state in the following way. We use the fact that the operator $e^{-\beta \bar{H}}$ is a solution of the Bloch equation :

$$
\frac{d}{d u} e^{-u \bar{H}}=-\bar{H} e^{-u \bar{H}}, 0<u<\beta
$$

Following the general method of [6], [13] to evaluate variationally a given quantity, we introduce an ansatz operator $\mathcal{D}(t)$ and we consider equation (3.2) as a constraint for the complex times $t=t_{0}+i(\beta-u)$ with the boundary condition $\mathcal{D}\left(t_{0}+i \beta\right)=1$. We introduce also a Lagrange multiplier $\mathcal{A}(t)$ associated to this constraint. The functional to minimize writes :

$\mathcal{Z}(\mathcal{A}(t), \mathcal{D}(t))=\operatorname{Tr}\left(\mathcal{A}\left(t_{0}+i 0\right) \mathcal{D}\left(t_{0}+i 0\right)\right)-\operatorname{Tr} \int_{t_{0}}^{t_{0}+i \beta} d t \mathcal{A}(t)\left(\frac{d \mathcal{D}(t)}{d t}+i \bar{H} \mathcal{D}(t)\right)+\mathcal{Z}_{d y n}$

where $\mathcal{Z}_{d y n}$ is given by expression (2.15) of section 2. The ansatz operators $\mathcal{D}(t)$ and $\mathcal{A}(t)$ are subject to the boundary conditions $\mathcal{A}(\infty)=1$ and $\mathcal{D}\left(t_{0}+i \beta\right)=1$.

Let us note that for a complex value of $t, \mathcal{A}(t)$ is not close to 1 in the limit of vanishing sources. Indeed, the stationarity condition of $\mathcal{Z}$ with respect to arbitrary variations of $\mathcal{D}(t)$ writes :

$$
\frac{d \mathcal{A}(t)}{d t}-i \mathcal{A}(t) \bar{H}=0
$$

and the solution of this equation for $t=t_{0}+i(\beta-u)$ is equal to : $\mathcal{A}(t)=A\left(t_{0}\right) \exp (-(\beta-$ u) $\bar{H})$.

The approximate dynamical equation for $\mathcal{D}(t)$ and $\mathcal{A}(t)$ are coupled. $\mathcal{D}\left(t_{0}\right)$ depends on $\mathcal{A}(t)$ for $t>t_{0}$ and therefore on the sources $J$ contrary to the exact state $D\left(t_{0}\right)$. 


\subsection{The variational spaces}

For the variational spaces for $\mathcal{D}(t)$ and $\mathcal{A}(t)$, we choose again Gaussian operators characterized by the contractions $\alpha_{d}(\mathbf{x}, t), \Xi_{d}(\mathbf{x}, \mathbf{y}, t)$ and $\alpha_{a}(\mathbf{x}, t), \Xi_{a}(\mathbf{x}, \mathbf{y}, t)$ where the time $t$ is on the two segments $\left[t_{0}+i \beta, t_{0}\left[\right.\right.$ and $\left[t_{0},+\infty[\right.$ in the complex plane.

The boundary condition $\mathcal{D}\left(t_{0}+i \beta\right)=1$ writes :

$$
\Xi_{d}^{-1}\left(t_{0}+i \beta\right)=0 \quad, \quad \frac{1}{\Xi_{d}} \alpha_{d}\left(t_{0}+i \beta\right)=0 .
$$

Let us write the functional $\mathcal{Z}(3.3)$ with the elementary contractions :

$\mathcal{Z}\left(n_{a}, \alpha_{a}, \Xi_{a} ; n_{d}, \alpha_{d}, \Xi_{d}\right)=n\left(t_{0}\right)-\int_{t_{0}+i \beta}^{t_{0}} d t\left[\left.\frac{d n(t)}{d t}\right|_{\mathcal{A}(t)=c t e}+i n(t) \int d^{3} x \overline{\mathcal{E}}_{c}(\mathbf{x}, t)\right]+\mathcal{Z}_{d y n}$,

or, by integrating by part :

$\mathcal{Z}\left(n_{a}, \alpha_{a}, \Xi_{a} ; n_{d}, \alpha_{d}, \Xi_{d}\right)=n\left(t_{0}+i \beta\right)+\int_{t_{0}+i \beta}^{t_{0}} d t\left[\left.\frac{d n(t)}{d t}\right|_{\mathcal{D}(t)=c t e}-i n(t) \int d^{3} x \overline{\mathcal{E}}_{c}(\mathbf{x}, t)\right]+\mathcal{Z}_{d y n}$

We have used the notation :

$$
\int d^{3} x \overline{\mathcal{E}}_{c}(\mathbf{x}, t)=\operatorname{Tr}(\mathcal{D}(t) \mathcal{A}(t) \bar{H})=<\bar{H}>_{c}
$$

\subsection{The dynamical equations}

For complex values of the time $t_{0}+i \beta<t<t_{0}$, the dynamical equations for the contractions $\alpha_{d}, \Xi_{d}, \alpha_{a}$ and $\Xi_{a}$ write :

$$
\begin{gathered}
2 i \dot{\alpha}_{d}=-\left(\Xi_{d}+\tau\right) \overline{\mathcal{H}}_{c}\left(\Xi_{d}-\tau\right) \tau \alpha_{b-c}+\left(\Xi_{d}+\tau\right) \bar{w}_{c}, \\
i \dot{\Xi}_{d}=-\left(\Xi_{d}+\tau\right) \overline{\mathcal{H}}_{c}\left(\Xi_{d}-\tau\right), \\
2 i \dot{\alpha}_{a}=\left(\Xi_{a}-\tau\right) \overline{\mathcal{H}}_{c}\left(\Xi_{a}+\tau\right) \tau \alpha_{c-b}-\left(\Xi_{a}-\tau\right) \bar{w}_{c}, \\
i \dot{\Xi}_{a}=\left(\Xi_{a}-\tau\right) \overline{\mathcal{H}}_{c}\left(\Xi_{a}+\tau\right) .
\end{gathered}
$$

These dynamical equations are coupled through the boundary conditions $\mathcal{D}\left(t_{0}+i \beta\right)=$ $1, \mathcal{A}(t=+\infty)=1$ which write :

$$
\begin{gathered}
\Xi_{d}^{-1}\left(t_{0}+i \beta\right)=0, \frac{1}{\Xi_{d}} \alpha_{d}\left(t_{0}+i \beta\right)=0, \\
\Xi_{a}^{-1}(t=+\infty)=0, \frac{1}{\Xi_{a}} \alpha_{a}(t=+\infty)=0 .
\end{gathered}
$$


For the contractions $\alpha_{b}, \Xi_{b}$ et $\alpha_{c}, \Xi_{c}$, the dynamical equation write :

$$
\begin{gathered}
\dot{\alpha}_{b}=0, \\
\dot{\Xi}_{b}=0, \\
i \dot{\alpha}_{c}=\tau \bar{w}_{c}, \\
i \dot{\Xi}_{c}=2\left(\Xi_{c} \overline{\mathcal{H}}_{c} \tau-\tau \overline{\mathcal{H}}_{c} \Xi_{c}\right) .
\end{gathered}
$$

We have $\frac{d<\bar{H}>c}{d t}=0$. We have introduce the vector $\bar{w}_{c}$ and the matrix $\overline{\mathcal{H}}_{c}$ (analogous to $w$ and $\mathcal{H}$ ) to express the variation $\delta<\bar{H}>$ in terms of the variations $\delta \alpha_{c}$ and $\delta \Xi_{c}$. All the contractions $\alpha$ et $\Xi$ are continous at $t=t_{0}$. In the complex time interval $\left[t_{0}+i \beta, t_{0}\right]$, $\alpha_{b}$ and $\Xi_{b}$ are constant $: \alpha_{b}=\alpha_{b}\left(t_{0}\right), \Xi_{b}=\Xi_{b}\left(t_{0}\right)$.

\subsection{Expansion in powers of the sources}

We use the index ${ }^{(0)}$ for the solutions of the dynamical equations in the limit with no source $: \alpha_{d}(t)=\alpha_{d}^{(0)}(t), \Xi_{d}(t)=\Xi_{d}^{(0)}(t), \alpha_{a}(t)=\alpha_{a}^{(0)}(t), \Xi_{a}(t)=\Xi_{a}^{(0)}(t)$. Since $\mathcal{A}(t) \neq 1$ even in the limit with no source, this limit does not correspond to $\frac{1}{\Xi_{a}} \alpha_{a}=0, \Xi_{a}^{-1}=0$, contrary to what happens when we do not optimize the initial state (see section 2). Therefore, $\Xi_{b}^{(0)} \neq \Xi_{d}^{(0)}$ and $\alpha_{b}^{(0)} \neq \alpha_{d}^{(0)}$. However, we can use a continuity property at $t=t_{0}$. in the limit with no source, $\mathcal{A}\left(t_{0}+i 0\right)=\mathcal{A}\left(t_{0}+0\right) \rightarrow 1$. At the time $t_{0}$, we thus have :

$$
\begin{aligned}
& \alpha_{b}^{(0)}\left(t_{0}\right)=\alpha_{c}^{(0)}\left(t_{0}\right)=\alpha_{d}^{(0)}\left(t_{0}\right)=\bar{\alpha}_{0}, \\
& \Xi_{b}^{(0)}\left(t_{0}\right)=\Xi_{c}^{(0)}\left(t_{0}\right)=\Xi_{d}^{(0)}\left(t_{0}\right)=\bar{\Xi}_{0},
\end{aligned}
$$

where $\bar{\alpha}_{0}$ and $\bar{\Xi}_{0}$ are the HB static solutions associated to the operator $\bar{H}$ and verify :

$$
\begin{gathered}
\bar{w}_{0}=0, \\
\bar{\Xi}_{0} \overline{\mathcal{H}}_{0} \tau=\tau \overline{\mathcal{H}}_{0} \bar{\Xi}_{0} .
\end{gathered}
$$

$\bar{\Xi}_{0}$ et $\overline{\mathcal{H}}_{0}$ are related by equations similar to (2.55) and (2.56). The expressions for the vector $\bar{w}_{0}=\bar{w}\left(\bar{\alpha}_{0}, \bar{\Xi}_{0}\right)$ and the matrix $\overline{\mathcal{H}}_{0}=\overline{\mathcal{H}}\left(\bar{\alpha}_{0}, \bar{\Xi}_{0}\right)$ are obtained from those in Appendix D by replacing $f_{0}$ and $g_{0}$ by the time-independent functions $\bar{f}_{0}$ and $\bar{g}_{0}$. (We recall that $\overline{\mathcal{H}}$ comes from the variation of $\langle\bar{H}>$ with respect to $\Xi$ and has to be evaluated at $\bar{\alpha}_{0}, \bar{\Xi}_{0}$ to obtain $\overline{\mathcal{H}}_{0}$. Since $\bar{H}$ is a time-independent operator, $\overline{\mathcal{H}}_{0}$ is time-independent.) We stress the difference between the quantities $\Xi^{(0)}, \Xi_{0}$ and $\bar{\Xi}_{0}$. 
The expansion at zero order of equations (3.9)-(3.12) writes :

$$
\begin{gathered}
2 i \dot{\alpha}_{d}^{(0)}=\left(\Xi_{d}^{(0)}+\tau\right) \bar{w}_{0}, \\
i \dot{\Xi}_{d}^{(0)}=-\left(\Xi_{d}^{(0)}+\tau\right) \overline{\mathcal{H}}_{0}\left(\Xi_{d}^{(0)}-\tau\right), \\
2 i \dot{\alpha}_{a}^{(0)}=\left(\Xi_{a}^{(0)}-\tau\right) \bar{w}_{0}, \\
i \dot{\Xi}_{a}^{(0)}=-\left(\Xi_{a}^{(0)}-\tau\right) \overline{\mathcal{H}}_{0}\left(\Xi_{a}^{(0)}+\tau\right) .
\end{gathered}
$$

Since $\bar{w}_{0}=0$, we have for $t_{0}+i \beta<t<t_{0}: \dot{\alpha}_{d}^{(0)}=0$ and $\dot{\alpha}_{a}^{(0)}=0$. By using the continuity at $t=t_{0}$, we thus obtain for $0<u<\beta$ :

$$
\begin{gathered}
\alpha_{d}^{(0)}\left(t_{0}+i \beta-i u\right)=\bar{\alpha}_{0} . \\
\left(\frac{1}{\Xi_{a}} \alpha_{a}\right)^{(0)}\left(t_{0}+i \beta-i u\right)=0
\end{gathered}
$$

The solution of (3.24) with the boundary condition $\Xi_{d}^{(0)^{-1}}\left(t_{0}+i \beta\right)=0$ is the following

$$
\Xi_{d}^{(0)}\left(t_{0}+i \beta-i u\right)=-\tau \operatorname{coth}\left(u \overline{\mathcal{H}}_{0} \tau\right)
$$

which can also be written as :

$$
\Xi_{d}^{(0)}\left(t_{0}+i \beta-i u\right)=\tau\left(1-\frac{2}{1-\exp \left(-u \overline{\mathcal{H}}_{0} 2 \tau\right)}\right)
$$

or in the uniform case :

$$
\Xi_{d}^{(0)}\left(t_{0}+i \beta-i u\right)=\left(\begin{array}{cc}
\frac{1}{\omega_{\mathbf{p}}} \operatorname{coth}\left(\frac{u \omega_{\mathbf{p}}}{2}\right) & 0 \\
0 & -\omega_{\mathbf{p}} \operatorname{coth}\left(\frac{u \omega_{\mathbf{p}}}{2}\right)
\end{array}\right)
$$

with, for the $\Phi^{4}$ theorie, $\omega_{\mathbf{p}}=\sqrt{-\bar{g}_{0}(\mathbf{p})}$ and

$$
\bar{g}_{0}(\mathbf{p})=-\left(\mathbf{p}^{2}+\bar{m}_{0}^{2}+\frac{b}{2} \bar{\varphi}_{0}^{2}+\frac{b}{2} \bar{G}_{0}(\mathbf{x}, \mathbf{x})\right)
$$

At $u=\beta$, we have to obtain the static HB solution associated to the Hamiltonian $\bar{H}, \bar{\Xi}_{0}$

$$
\Xi_{d}^{(0)}\left(t_{0}+i 0\right)=\bar{\Xi}_{0}=-\tau \operatorname{coth}\left(\beta \overline{\mathcal{H}}_{0} \tau\right)
$$

The solution of 3.26$)$ writes :

$$
\Xi_{a}^{(0)}\left(t_{0}+i \beta-i u\right)=-\tau \operatorname{coth}\left((\beta-u) \overline{\mathcal{H}}_{0} \tau\right)
$$

One checks that for $u=\beta, \Xi_{a}^{(0)^{-1}}\left(t_{0}\right)=0$. 
At the first order, $\bar{w}_{c}^{(0)}\left(t_{0}\right)=\bar{w}_{0}\left(t_{0}\right)=0$ and $\bar{\Xi}_{c}^{(0)}\left(t_{0}\right)=\bar{\Xi}_{0}$. Therefore the right members of equations (3.17) and (3.18) are vanishing at $t_{0}$. We deduce that at the first order in the expansion in powers of the sources, $\alpha_{c}$ and $\Xi_{c}$ are constant in the interval $\left[t_{0}+\right.$ $\left.i \beta, t_{0}\right]$ and equal to $\bar{\alpha}_{0}$ and $\bar{\Xi}_{0}$ respectively. We can also obtain this result from the relations of Appendix $\mathrm{C}$ and from the expressions obtained above for $\alpha_{d}^{(0)}(t), \alpha_{a}^{(0)}(t), \Xi_{d}^{(0)}(t), \Xi_{a}^{(0)}(t)$.

At the next order, the approximated initial state characterized by $\alpha_{d}\left(t_{0}\right), \Xi_{d}\left(t_{0}\right)$ is no more equal to the static HB solution $\alpha_{0}, \Xi_{0}$.

Instead of using the expansion of equations (3.9) and (3.10) for $\alpha_{d}$ and $\Xi_{d}$, it is more convenient to expand at the first order equations (3.17) and (3.18) for $\alpha_{c}$ and $\Xi_{c}$. For $t_{0}+i \beta \leq t \leq t_{0}$ :

$$
\begin{aligned}
i \frac{d}{d t} \beta^{\Phi}\left(\vec{x}, \vec{y}, t, t^{\prime \prime}\right) & =\tau\left[\bar{t} \beta^{\Phi}\left(t, t^{\prime \prime}\right)-\frac{1}{2} \bar{T}_{,(j k} \Sigma_{k j)}^{\Phi}\left(t, t^{\prime \prime}\right)\right], \\
i \frac{d}{d t} \Sigma_{i j}^{\Phi}\left(\vec{x}, \vec{y}, \vec{x}_{1}, t, t^{\prime \prime}\right)= & 2\left[\Sigma^{\Phi} \overline{\mathcal{H}}_{0} \tau-\tau \overline{\mathcal{H}}_{0} \Sigma^{\Phi}\right. \\
& +\bar{\Xi}_{0} \bar{r}_{,(k} \beta_{k)}^{\Phi} \tau-\tau \bar{r}_{,(k} \beta_{k)}^{\Phi} \bar{\Xi}_{0} \\
& \left.-\frac{1}{2} \bar{\Xi}_{0} \bar{R}_{,(k l} \Sigma_{l k)}^{\Phi} \tau+\frac{1}{2} \tau \bar{R}_{,(k l} \Sigma_{l k)}^{\Phi} \bar{\Xi}_{0}\right]_{i j}
\end{aligned}
$$

The matrices $\bar{t}, \bar{T}$ and $\bar{r}, \bar{R}$ associated to the operator $\bar{H}$ and depending on $\bar{\Xi}_{0}$ and on $\bar{\alpha}_{0}$ are analogous to the matrices $t, T, r$ and $R$. The quantities $\overline{\mathcal{H}}_{0}, \bar{\alpha}_{0}, \bar{\Xi}_{0}$ are timeindependent for $t_{0}+i \beta \leq t \leq t_{0}$. The matrices $\bar{t}, \bar{T}$ and $\bar{r}, \bar{R}$ are also time-independent for $t_{0}+i \beta \leq t \leq t_{0}$. Indeed, these are defined from the expansion of $\bar{w}_{c}$ and $\overline{\mathcal{H}}_{c}$ in terms of the variations $\delta \alpha_{c}$ and $\delta \Xi_{c}$ (see equations (2.77) and (2.78)) and they have to be evaluated for the values $\alpha_{c}^{(0)}$ and $\Xi_{c}^{(0)}$. Now, as we have noticed before, these quantities do not depend on the complex time and are equal respectively to $\bar{\alpha}_{0}$ and $\bar{\Xi}_{0}$. For the same reason, $\bar{\Xi}_{0}$ appears in the last two lines of (3.36) and not $\Xi_{d}^{(0)}$ as in equation (2.80) which describes the evolution of $\Sigma^{\Phi}\left(t, t^{\prime \prime}\right)$ for real time.

\subsection{Approximation for the two-point function}

In the following we consider an uniform problem. In momentum space, equation (3.35) for $\beta^{\Phi}$ writes :

$$
\begin{gathered}
\frac{d^{2}}{d t^{2}} \beta_{1}^{\Phi}\left(\mathbf{p}, t, t^{\prime \prime}\right)=\bar{g}_{0}(-\mathbf{p}) \beta_{1}^{\Phi}\left(\mathbf{p}, t, t^{\prime \prime}\right)+\frac{b}{4} \bar{\varphi}_{0} \int_{\mathbf{p}_{1}+\mathbf{p}_{2}=-\mathbf{p}} \frac{d^{3} p_{1}}{(2 \pi)^{3}} \frac{d^{3} p_{2}}{(2 \pi)^{3}} \Sigma_{11}^{\Phi}\left(\mathbf{p}_{1}, \mathbf{p}_{2}, \mathbf{p}, t, t^{\prime \prime}\right) \\
i \frac{d}{d t} \beta_{1}^{\Phi}\left(\mathbf{p}, t, t^{\prime \prime}\right)=-\beta_{2}^{\Phi}\left(\mathbf{p}, t, t^{\prime \prime}\right)
\end{gathered}
$$




\section{Symmetric phase}

In the symmetric phase, $\bar{\varphi}_{0}=0$ and the equation for the function $\beta_{1}^{\Phi}\left(\mathbf{p}, t, t^{\prime \prime}\right)$ for $t_{0}+i \beta<t<t_{0}$ writes :

$$
\frac{d^{2}}{d t^{2}} \beta_{1}^{\Phi}\left(\mathbf{p}, t, t^{\prime \prime}\right)=\bar{g}_{0}(\mathbf{p}) \beta_{1}^{\Phi}\left(\mathbf{p}, t, t^{\prime \prime}\right)
$$

where

$$
-\bar{g}_{0}(\mathbf{p})=\mathbf{p}^{2}+\bar{m}_{0}^{2}+\frac{b}{2} \bar{G}_{0}(\mathbf{x}, \mathbf{x})
$$

We can therefore relate $\beta_{1,2}^{\Phi}\left(\mathbf{p}, t_{0}, t^{\prime \prime}\right)$ to $\beta_{1,2}^{\Phi}\left(\mathbf{p}, t_{0}+i \beta, t^{\prime \prime}\right)$ :

$$
\left(\begin{array}{c}
\beta_{1}^{\Phi} \\
\beta_{2}^{\Phi}
\end{array}\right)\left(\mathbf{p}, t_{0}, t^{\prime \prime}\right)=A(\mathbf{p})\left(\begin{array}{c}
\beta_{1}^{\Phi} \\
\beta_{2}^{\Phi}
\end{array}\right)\left(\mathbf{p}, t_{0}+i \beta, t^{\prime \prime}\right)
$$

where $A(\mathbf{p})$ is the matrix :

$$
A(\mathbf{p})=\left(\begin{array}{cc}
\cosh \omega_{\mathbf{p}} \beta & \frac{\sinh \omega_{\mathbf{p}} \beta}{\omega_{\mathbf{p}}} \\
\omega_{\mathbf{p}} \sinh \omega_{\mathbf{p}} \beta & \cosh \omega_{\mathbf{p}} \beta
\end{array}\right)
$$

with $\omega_{\mathbf{p}}=\sqrt{-\bar{g}_{0}(\mathbf{p})}$.

We then use the fact that $\mathcal{D}\left(t_{0}+i \beta\right)=1$. We deduce :

$$
\alpha_{c}\left(t_{0}+i \beta\right)=\alpha_{b}\left(t_{0}+i \beta\right) \quad, \quad \Xi_{c}\left(t_{0}+i \beta\right)=\Xi_{b}\left(t_{0}+i \beta\right) .
$$

By introducing the functions $\delta$ and $\Delta$ which come from the expansion at the first order in powers of the sources of $\alpha_{b}$ and $\Xi_{b}$ (and which are analogous to $\beta$ and $\Sigma$ ), we thus have :

$$
\begin{aligned}
\beta\left(t_{0}+i \beta, t^{\prime \prime}\right) & =\delta\left(t_{0}+i \beta, t^{\prime \prime}\right) \\
\Sigma\left(t_{0}+i \beta, t^{\prime \prime}\right) & =\Delta\left(t_{0}+i \beta, t^{\prime \prime}\right)
\end{aligned}
$$

Now $\alpha_{b}$ and $\Xi_{b}$ are constants for $t_{0}+i \beta<t<t_{0}$ (eq. (3.15) and (3.16)). Consequently :

$$
\begin{gathered}
\delta\left(t_{0}, t^{\prime \prime}\right)=\delta\left(t_{0}+i \beta, t^{\prime \prime}\right) \\
\Delta\left(t_{0}, t^{\prime \prime}\right)=\Delta\left(t_{0}+i \beta, t^{\prime \prime}\right)
\end{gathered}
$$

Let us compare the expansion of $\Xi_{b}, \Xi_{c}$ and $\alpha_{b}, \alpha_{c}$ at $t_{0}$. By using equation (C.6) at $t=t_{0}$,

$$
\Delta_{k}\left(t_{0}, t^{\prime \prime}\right)=-\left(\bar{\Xi}_{0}+\tau\right) l_{k}\left(t^{\prime \prime}, t_{0}\right)\left(\bar{\Xi}_{0}-\tau\right)
$$

and from equation (C.1) :

$$
\delta_{k}\left(t_{0}, t^{\prime \prime}\right)=\left(\bar{\Xi}_{0}+\tau\right)\left(m_{k}\left(t^{\prime \prime}, t_{0}\right)-l_{k}\left(t^{\prime \prime}, t_{0}\right) \bar{\alpha}_{0}\right) .
$$


The comparison of these last two equations to (2.86) and (2.87) leads to :

$$
\begin{gathered}
\delta_{k}\left(t_{0}, t^{\prime \prime}\right)=\beta_{k}\left(t_{0}, t^{\prime \prime}\right)+2 \tau\left(m_{k}\left(t^{\prime \prime}, t_{0}\right)-l_{k}\left(t^{\prime \prime}, t_{0}\right) \alpha_{0}\right) \\
\Delta_{k}\left(t_{0}, t^{\prime \prime}\right)=\Sigma_{k}\left(t_{0}, t^{\prime \prime}\right)+2 \bar{\Xi}_{0} l_{k}\left(t^{\prime \prime}, t_{0}\right) \tau-2 \tau l_{k}\left(t^{\prime \prime}, t_{0}\right) \bar{\Xi}_{0} .
\end{gathered}
$$

Finally, we use :

$$
\begin{aligned}
\beta\left(t_{0}+i \beta, t^{\prime \prime}\right) & =\delta\left(t_{0}, t^{\prime \prime}\right) \\
\Sigma\left(t_{0}+i \beta, t^{\prime \prime}\right) & =\Delta\left(t_{0}, t^{\prime \prime}\right)
\end{aligned}
$$

In the symmetric phase, $l^{\Phi}=0$, therefore :

$$
\beta^{\Phi}\left(t_{0}+i \beta, t^{\prime \prime}\right)=\beta^{\Phi}\left(t_{0}, t^{\prime \prime}\right)+2 \tau m^{\Phi}\left(t^{\prime \prime}, t_{0}\right)
$$

From equation (3.41), we obtain :

$$
\beta^{\Phi}\left(\mathbf{p}, t_{0}, t^{\prime \prime}\right)=\left(A^{-1}(\mathbf{p})-1\right)^{-1} 2 \tau m^{\Phi}\left(\mathbf{p}, t^{\prime \prime}, t_{0}\right)
$$

For $t^{\prime \prime}>t^{\prime}$, the relation (2.109) of section 2 is therefore modified according to :

$$
\beta_{1}^{\Phi}\left(\mathbf{p}, t^{\prime}, t^{\prime \prime}\right)=4 \tilde{m}^{\Phi}\left(\mathbf{p}, t^{\prime}, t_{0}\right)\left(A^{-1}(\mathbf{p})-1\right)^{-1} \tau m^{\Phi}\left(\mathbf{p}, t^{\prime \prime}, t_{0}\right)
$$

with

For $t^{\prime}>t^{\prime \prime}$, we have :

$$
\left(A^{-1}(\mathbf{p})-1\right)^{-1}=\left(\begin{array}{cc}
-\frac{1}{2} & -\frac{1}{2 \omega_{\mathbf{p}}} \operatorname{coth}\left(\frac{\beta}{2} \omega_{\mathbf{p}}\right) \\
-\frac{\omega_{\mathbf{p}}}{2} \operatorname{coth}\left(\frac{\beta}{2} \omega_{\mathbf{p}}\right) & -\frac{1}{2}
\end{array}\right)
$$

$$
\beta_{1}^{\Phi}\left(\mathbf{p}, t^{\prime}, t^{\prime \prime}\right)=4 \tilde{m}^{\Phi}\left(\mathbf{p}, t^{\prime}, t_{0}\right)\left(\left(A^{-1}(\mathbf{p})-1\right)^{-1}+1\right) \tau m^{\Phi}\left(\mathbf{p}, t^{\prime \prime}, t_{0}\right)
$$

We check again that in the limit $t^{\prime}=t^{\prime \prime}=t_{0}$, we obtain the TDHB solution at $t_{0}$ : $\beta_{1}^{\Phi}\left(\mathbf{p}, t_{0}, t_{0}\right)=\frac{1}{2 \omega_{\mathbf{p}}} \operatorname{coth}\left(\beta \omega_{\mathbf{p}}\right)=G_{0}\left(\mathbf{p}, t_{0}\right)$.

For the two-point function in the symmetric phase, one can check from expression (2.57) for the matrix $\bar{\Xi}_{0}$ (and where the quantities which appear correspond now to $\bar{H}$ and no more $H$ ) that :

$$
\bar{\Xi}_{0}(p)-\tau=2\left(A^{-1}(p)-1\right)^{-1} \tau
$$

Therefore expressions (2.109) and (3.56) for $\beta_{1}^{\Phi}$ in the symmetric phase obtained without or with optimization with respect to the initial state coïncide when $\bar{\Xi}_{0}=\Xi_{0}$ which is the case at equilibrium. This is not true in the asymmetric phase. It will be also more interesting to study the four-point function in section 3.6.

It is also interesting to calculate the approximation obtained for the retarded Green function (2.115) when we optimize both the dynamics and the initial state. In the symmetric phase, we have for the time-ordered correlation function for $t^{\prime}>t^{\prime \prime}$ :

$$
\beta_{1}^{\Phi}\left(t^{\prime}, t^{\prime \prime}\right)=2 \tilde{m}^{\Phi}\left(t^{\prime \prime}, t_{0}\right) \beta^{\Phi}\left(t_{0}, t^{\prime}\right)
$$


For the anti-T product and $t^{\prime}>t^{\prime \prime}$ :

$$
\beta_{1}^{\Phi}\left(t^{\prime}, t^{\prime \prime}\right)=2 \tilde{m}^{\Phi}\left(t^{\prime}, t_{0}\right) \beta^{\Phi}\left(t_{0}, t^{\prime \prime}\right)
$$

By using (3.55) and the fact that

$$
\left(A^{-1}(p)-1\right)^{-1} \tau+\left[\tau\left(A^{-1}(p)-1\right)^{-1}\right]^{T}=-\tau,
$$

we obtain :

$$
\chi_{\Phi \Phi}\left(p, t^{\prime}, t^{\prime \prime}\right)=i \theta\left(t^{\prime}-t^{\prime \prime}\right) 4 \tilde{m}^{\Phi}\left(p, t^{\prime \prime}, t_{0}\right) \tau m^{\Phi}\left(p, t^{\prime}, t_{0}\right)
$$

which is identical to the formula (2.117) obtained without the optimization of the initial state. For the retarded Green function the approach of section 2 is therefore sufficient.

\section{The case at equilibrium}

In this case, the two-time functions should depend only on the difference $t^{\prime}-t^{\prime \prime}$. Unfortunatly, this is not true for the expressions we gave for the three-point and fourpoint functions in section 2. At equilibrium, $\alpha^{(0)}(t)=\alpha_{0}, \Xi^{(0)}(t)=\Xi_{0}$ and the matrices $t, T, r, R$ are constant. In equations (2.89) and (2.90) for $l^{k}$ and $m^{k}$, the shift $t^{\prime} \rightarrow t^{\prime}+\delta t$ is therefore equivalent to a shift of the initial time $t_{0} \rightarrow t_{0}-\delta t$. By writing the corresponding shifts of the functions $l^{k}$ and $m^{k}-l^{k} \alpha_{0}$ and by inserting them in eq. (2.105) or in eq. (2.107), one sees that, in general, our approximations are not invariant under the shift $t^{\prime} \rightarrow t^{\prime}+\delta t, t^{\prime \prime} \rightarrow t^{\prime \prime}+\delta t, t_{0}$ being constant. They contain a spurious dependence on the initial time $t_{0}$.

The case of the two-point function in the symmetric phase is special. From equation (2.90) for $m^{\Phi}$, we have $\delta m^{\Phi}=i t \tau m^{\Phi} \delta t$ and the variation of expression 2.109) is equal to zero because $\delta \beta_{1}^{\Phi}$ is proportional to $-\Xi_{11}^{0} g_{0}+\Xi_{22}^{0}$ which is vanishing for the static solution. However, this is no more true for the four-point function $\Sigma^{\Phi \Phi}$ given by equation (2.113). The variation $\delta \Sigma$ involves two types of terms : one with the matrix $R$ proportional to the coupling constant and another with the matrix $\mathcal{H}_{0}$. Generally, $\delta \Sigma$ is non vanishing. This spurious dependence on the initial time will disappear when we take into account the optimization with respect to the initial state : the two-time correlation functions depend only on the time difference $t^{\prime}-t^{\prime \prime}$ as they should.

At equilibrium, in the symmetric phase, we can solve equation (2.90) for $m^{\Phi}$ :

$$
\begin{gathered}
m_{1}^{\Phi}\left(\mathbf{p}, t^{\prime}, t_{0}\right)=-\frac{1}{2} \cos \left(\omega_{\mathbf{p}}\left(t_{0}-t^{\prime}\right)\right) \\
m_{2}^{\Phi}\left(\mathbf{p}, t^{\prime}, t_{0}\right)=-\frac{1}{2 i} \frac{1}{\omega_{\mathbf{p}}} \sin \left(\omega_{\mathbf{p}}\left(t_{0}-t^{\prime}\right)\right)
\end{gathered}
$$

We define $\Delta t=t^{\prime}-t^{\prime \prime}$. For $\Delta t<0$, equation (3.56) gives

$$
\beta_{1}^{\Phi}(\mathbf{p}, \Delta t)=\frac{1}{2 \omega_{\mathbf{p}}}\left(\operatorname{coth} \frac{\beta \omega_{\mathbf{p}}}{2} \cos \omega_{\mathbf{p}} \Delta t-\frac{1}{i} \sin \omega_{\mathbf{p}} \Delta t\right)
$$


For $\Delta t>0$, we use equation $(3.58)$ :

$$
\beta_{1}^{\Phi}(\mathbf{p}, \Delta t)=\frac{1}{2 \omega_{\mathbf{p}}}\left(\operatorname{coth} \frac{\beta \omega_{\mathbf{p}}}{2} \cos \omega_{\mathbf{p}} \Delta t+\frac{1}{i} \sin \omega_{\mathbf{p}} \Delta t\right)
$$

We check that $\beta_{1}^{\Phi}$ depends only on $\Delta t=t^{\prime}-t^{\prime \prime}$. Expressions (3.66) and (3.67) are interchanged by changing $\Delta t$ to $-\Delta t$, which was not explicit on formula (3.56) and (3.58).

For $\Delta t=0$, we obtain $\beta_{1}^{\Phi}(\mathbf{p}, \Delta t=0)=G_{0}(\mathbf{p})$. In this case, at equal time, the twotime and two-point function coïncides with the mean-field HB solution for the two-point function.

By taking the Fourrier transform in time of equations (3.66) and (3.67) :

$$
\beta_{1}^{\Phi}(\mathbf{p}, \omega)=\int_{-\infty}^{0} d(\Delta t) e^{i(\omega-i \epsilon) \Delta t} \beta_{1}^{\Phi}(\mathbf{p}, \Delta t)+\int_{0}^{+\infty} d(\Delta t) e^{i(\omega+i \epsilon) \Delta t} \beta_{1}^{\Phi}(\mathbf{p}, \Delta t),
$$

we obtain :

$$
\beta_{1}^{\Phi}(\mathbf{p}, \omega)=i\left[\frac{n_{\mathbf{p}}+1}{\omega^{2}-\left(\omega_{\mathbf{p}}-i \epsilon\right)^{2}}-\frac{n_{\mathbf{p}}}{\omega^{2}-\left(\omega_{\mathbf{p}}+i \epsilon\right)^{2}}\right]
$$

where $n_{\mathbf{p}}$ is the occupation number : $2 n_{\mathbf{p}}+1=\operatorname{coth} \frac{\beta \omega_{\mathbf{p}}}{2}$.

We will now show that our approximation for the two-time correlation function with two field operators satisfies to the fluctuation-dissipation theorem. This theorem can be written as a relation between the Fourrier transform in time of the correlation function $C_{\Phi \Phi}^{2}\left(\mathbf{x}, \mathbf{x}^{\prime}, t, t^{\prime}\right)$ and the response function

$$
\chi_{2}\left(\mathbf{x}, \mathbf{x}^{\prime}, t, t^{\prime}\right)=\operatorname{Tr}\left(\left[\Phi^{H}\left(\mathbf{x}, t, t_{0}\right), \Phi^{H}\left(\mathbf{x}^{\prime}, t^{\prime}, t_{0}\right)\right] D\left(t_{0}\right)\right)
$$

in the following way :

$$
\tilde{\chi}_{2}\left(\mathbf{x}, \mathbf{x}^{\prime}, \omega\right)=\left(1-e^{-\beta \omega}\right) \tilde{C}_{\Phi \Phi}^{2}\left(\mathbf{x}, \mathbf{x}^{\prime}, \omega\right)
$$

where the Fourrier transform in time is defined according to :

$$
\tilde{\chi}_{2}\left(\mathbf{x}, \mathbf{x}^{\prime}, \omega\right)=\int_{-\infty}^{+\infty} d\left(t-t^{\prime}\right) e^{i \omega\left(t-t^{\prime}\right)} \chi_{2}\left(\mathbf{x}, \mathbf{x}^{\prime}, t-t^{\prime}\right)
$$

The correlation function $C^{2}$ gives us informations about the fluctuations in the equilibrium state while the response function $\chi^{2}$ contains informations on the dynamics of the system which has been driven from the equilibrium by a small external perturbation. The function $\chi^{2}$ appears also in the dissipation energy of the system. Therefore, the fluctuation-disspation theorem tells us that, in systems near equilibrium, the transport properties which are linear in the external forces can be calculated from the fluctuations at equilibrium [20].

By using the definition

$$
\operatorname{Tr}\left(\Phi^{H}\left(\mathbf{x}, t^{\prime}, t_{0}\right) \Phi^{H}\left(\mathbf{y}, t^{\prime \prime}, t_{0}\right) D\left(t_{0}\right)\right)-\varphi\left(\mathbf{x}, t^{\prime}\right) \varphi\left(\mathbf{y}, t^{\prime \prime}\right) \equiv<\Phi(\mathbf{x}, \tau) \Phi(\mathbf{y}, \tau)>
$$


with $\tau=t^{\prime}-t^{\prime \prime}$, the fluctuation-dissipation theorem can be written in the following way :

$$
\begin{aligned}
\int_{-\infty}^{+\infty} d \tau e^{i \omega \tau}<\Phi(\mathbf{x}, \tau) \Phi(\mathbf{y})>= & e^{\beta \omega} \int_{-\infty}^{+\infty} d \tau e^{i \omega \tau}<\Phi(\mathbf{y}) \Phi(\mathbf{x}, \tau)> \\
& =\int_{-\infty}^{+\infty} d \tau e^{i \omega(\tau-i \beta)}<\Phi(\mathbf{x}, \tau-i \beta) \Phi(\mathbf{y})>
\end{aligned}
$$

In order to check the fluctuation-dissipation theorem on our approximations for the twopoint correlation function, it is therefore sufficient to check that expression (3.66) for $\Delta t=\tau$ is equivalent to expression (3.67) for $\Delta t=-\tau-i \beta$, which can be done easely.

At equilibrium, expression (2.117) for the retarded Green function with two field operators in the symmetric phase gives :

$$
\chi_{\Phi \Phi}\left(\mathbf{p}, t^{\prime}, t^{\prime \prime}\right)=-\theta\left(t^{\prime}-t^{\prime \prime}\right) \frac{1}{\omega_{\mathbf{p}}} \sin \omega_{\mathbf{p}}\left(t^{\prime}-t^{\prime \prime}\right)
$$

\subsection{Four-point function : a perturbative expansion}

Still in the symmetric phase and for the uniform case, we consider now the four-point function $\Sigma^{\Phi \Phi}$.

\subsubsection{The response function $\Pi_{R}\left(\mathbf{q}^{2}, t^{\prime}, t^{\prime \prime}\right)$}

When one couples a source $J^{\Phi \Phi}\left(\mathbf{x}, \mathbf{y}, t^{\prime}\right)$ to the the operator $\Phi^{H}\left(\mathbf{x}, t^{\prime}, t_{0}\right) \Phi^{H}\left(\mathbf{y}, t^{\prime \prime}, t_{0}\right)$, the linear response theory gives the variation of $G\left(\mathbf{x}, \mathbf{y}, t^{\prime}\right)=\operatorname{Tr}\left(\Phi^{H}\left(\mathbf{x}, t^{\prime}, t_{0}\right) \Phi^{H}\left(\mathbf{y}, t^{\prime}, t_{0}\right) D\left(t_{0}\right)\right)$ in presence of the source :

$$
\delta G\left(\mathbf{x}, \mathbf{y}, t^{\prime}\right)=\int_{t_{0}}^{+\infty} d t^{\prime \prime} \int d^{3} z d^{3} u \chi_{\Phi \Phi, \Phi \Phi}\left(\mathbf{x}, \mathbf{y}, \mathbf{z}, \mathbf{u}, t^{\prime}, t^{\prime \prime}\right) J^{\Phi \Phi}\left(\mathbf{z}, \mathbf{u}, t^{\prime \prime}\right)
$$

where $\chi_{\Phi \Phi, \Phi \Phi}$ is the retarded Green function with four field operators defined in eq. (2.120). In presence of a source of the following type :

$$
\begin{gathered}
J^{\Phi \Phi}\left(\mathbf{x}_{1}, \mathbf{x}_{2}, t^{\prime \prime}\right)=J^{\Phi \Phi}\left(t^{\prime \prime}\right) e^{-i \mathbf{q} \cdot \mathbf{x}_{1}} \delta^{3}\left(\mathbf{x}_{1}-\mathbf{x}_{2}\right) \\
\delta G\left(\mathbf{x}, \mathbf{y}, t^{\prime}\right)=\int_{t_{0}}^{+\infty} d t^{\prime \prime} J^{\Phi \Phi}\left(t^{\prime \prime}\right) \int d^{3} z e^{-i \mathbf{q} \cdot \mathbf{z}} \chi_{\Phi \Phi, \Phi \Phi}\left(\mathbf{x}, \mathbf{y}, \mathbf{z}, \mathbf{z}, t^{\prime}, t^{\prime \prime}\right)
\end{gathered}
$$

By introducing the Fourrier transform, we obtain for $\mathbf{x}=\mathbf{y}$ :

$\delta G\left(\mathbf{x}, \mathbf{x}, t^{\prime}\right)=e^{-i \mathbf{q} \cdot \mathbf{x}} \int d t^{\prime \prime} J^{\Phi \Phi}\left(t^{\prime \prime}\right) \int \frac{d^{3} k}{(2 \pi)^{3}} \frac{d^{3} p_{1}}{(2 \pi)^{3}} \chi_{\Phi \Phi, \Phi \Phi}\left(\mathbf{k},-\mathbf{q}-\mathbf{k}, \mathbf{p}_{1}, \mathbf{q}-\mathbf{p}_{1}, t^{\prime}, t^{\prime \prime}\right)$

The polarization function $\Pi_{R}$ is defined according to :

$$
\left.\Pi_{R}\left(\mathbf{q}^{2}, t^{\prime}, t^{\prime \prime}\right) \equiv \frac{\delta G\left(\mathbf{x}, \mathbf{x}, t^{\prime}\right)}{e^{-i \mathbf{q} \cdot \mathbf{x}} \delta J^{\Phi \Phi}\left(t^{\prime \prime}\right)}\right|_{J^{\Phi \Phi}=0}
$$


i. e. :

$$
\Pi_{R}\left(\mathbf{q}^{2}, t^{\prime}, t^{\prime \prime}\right)=\int \frac{d^{3} k}{(2 \pi)^{3}} \frac{d^{3} p_{1}}{(2 \pi)^{3}} \chi_{\Phi \Phi, \Phi \Phi}\left(\mathbf{k},-\mathbf{q}-\mathbf{k}, \mathbf{p}_{1}, \mathbf{q}-\mathbf{p}_{1}, t^{\prime}, t^{\prime \prime}\right)
$$

A variational approximation for the polarization function $\Pi_{R}$ is obtained by using expression (2.121) for $\chi_{\Phi \Phi, \Phi \Phi}$ as a function of $l^{\Phi \Phi}$ and $\Xi_{0}$.

In the static case, $\Pi_{R}\left(\mathbf{q}^{2}, t^{\prime}, t^{\prime \prime}\right)$ depends only on $\Delta t=t^{\prime}-t^{\prime \prime}$ and is proportional to $\theta\left(t^{\prime}-t^{\prime \prime}\right)$. We thus define its Fourrier transform in time by :

$$
\tilde{\Pi}_{R}\left(\mathbf{q}^{2}, \omega\right)=\int_{0}^{+\infty} d(\Delta t) e^{i \omega \Delta t+\epsilon \Delta t} \Pi_{R}\left(\mathbf{q}^{2}, \Delta t\right)
$$

The polarization function $\Pi_{R}\left(\mathbf{q}^{2}, t^{\prime}, t^{\prime \prime}\right)$ can be recognized as the Fourrier transform with respect to $\mathbf{x}-\mathbf{y}$ of the retarded Green function for the operators $\Phi^{2}(\mathbf{x})$ and $\Phi^{2}(\mathbf{y})$ : $\chi_{\Phi^{2}, \Phi^{2}}\left(\mathbf{x}, \mathbf{x}, \mathbf{y}, \mathbf{y}, t^{\prime}, t^{\prime \prime}\right)=-i \theta\left(t^{\prime}-t^{\prime \prime}\right) \operatorname{Tr}\left(\left[\Phi^{H}\left(\mathbf{x}, t^{\prime}, t_{0}\right) \Phi^{H}\left(\mathbf{x}, t^{\prime}, t_{0}\right), \Phi^{H}\left(\mathbf{y}, t^{\prime \prime}, t_{0}\right) \Phi^{H}\left(\mathbf{y}, t^{\prime \prime}, t_{0}\right)\right] D\left(t_{0}\right)\right)$

We have :

$$
\begin{aligned}
\chi_{\Phi^{2}, \Phi^{2}}\left(\mathbf{q}^{2}, t^{\prime}, t^{\prime \prime}\right)= & \int d^{3} x e^{-i \mathbf{q} \cdot(\mathbf{x}-\mathbf{y})} \chi_{\Phi^{2}, \Phi^{2}}\left(\mathbf{x}, \mathbf{x}, \mathbf{y}, \mathbf{y}, t^{\prime}, t^{\prime \prime}\right) \\
& =\Pi_{R}\left(\mathbf{q}^{2}, t^{\prime}, t^{\prime \prime}\right)
\end{aligned}
$$

This function is directly related to the viscuosity in the $\Phi^{4}$ theory. From the spectral representation :

$$
\chi_{\Phi^{2}, \Phi^{2}}\left(\mathbf{p}, p_{0}\right)=\lim _{\epsilon \rightarrow 0^{+}} \int_{-\infty}^{+\infty} d \omega \frac{\rho_{\Phi^{2}, \Phi^{2}}(\mathbf{p}, \omega)}{p_{0}-\omega+i \epsilon},
$$

the viscuosity is defined by :

$$
\eta_{\Phi^{2}, \Phi^{2}} \equiv \lim _{\mathbf{p}, p_{0} \rightarrow 0}\left[\frac{\rho_{\Phi^{2}, \Phi^{2}}\left(\mathbf{p}, p_{0}\right)}{p_{0}}\right]
$$

Calculations of $\eta_{\Phi^{2}, \Phi^{2}}$ have been done in the limit of high temperature and the result have been compared to the classical approximation [21].

Actually we will begin by calculating the following non-retarded Green function :

$$
\left.\Pi\left(\mathbf{q}^{2}, t^{\prime}, t^{\prime \prime}\right) \equiv \frac{1}{2} \frac{\delta \Xi_{11}^{c}\left(\mathbf{x}, \mathbf{x}, t^{\prime}\right)}{e^{-i \mathbf{q} \cdot \mathbf{x}} \delta J^{\Phi \Phi}\left(t^{\prime \prime}\right)}\right|_{J^{\Phi \Phi}=0}
$$

which can be written with the four-point function $\Sigma_{11}^{\Phi \Phi}$ as :

$$
\Pi\left(\mathbf{q}^{2}, t^{\prime}, t^{\prime \prime}\right)=\frac{i}{2} \int \frac{d^{3} k}{(2 \pi)^{3}} \int \frac{d^{3} p_{1}}{(2 \pi)^{3}} \Sigma_{11}^{\Phi \Phi}\left(\mathbf{k},-\mathbf{q}-\mathbf{k}, \mathbf{p}_{1}, \mathbf{q}-\mathbf{p}_{1}, t^{\prime}, t^{\prime \prime}\right)
$$

In the following, we will therefore consider the function $\Sigma^{\Phi \Phi}\left(\mathbf{k},-\mathbf{q}-\mathbf{k}, \mathbf{p}_{1}, \mathbf{q}-\mathbf{p}_{1}, t^{\prime}, t^{\prime \prime}\right)$. We will show the differences between the approximations for the function $\Pi\left(\mathbf{q}^{2}, t^{\prime}, t^{\prime \prime}\right)$ when we do or do not optimize with respect to the initial state. 


\subsubsection{Resolution of the dynamical equations for complex values of the time}

In the symmetric phase, we have for $t_{0}+i \beta \leq t \leq t_{0}$ :

$$
\begin{gathered}
i \frac{d}{d t} \sum_{11}^{\Phi \Phi}\left(\mathbf{p}_{1}, \mathbf{p}_{2}, \mathbf{p}_{3},-\sum \mathbf{p}_{i}, t, t^{\prime \prime}\right)=-\Sigma_{12}^{\Phi \Phi}-\Sigma_{21}^{\Phi \Phi} \\
i \frac{d}{d t} \sum_{12}^{\Phi \Phi}=\bar{g}_{0}\left(-p_{2}\right) \sum_{11}^{\Phi \Phi}-\Sigma_{22}^{\Phi \Phi}-\frac{b}{4} \bar{\Xi}_{11}^{0}\left(-p_{1}\right) I \\
i \frac{d}{d t} \sum_{21}^{\Phi \Phi}=\bar{g}_{0}\left(p_{1}\right) \Sigma_{11}^{\Phi \Phi}-\Sigma_{22}^{\Phi \Phi}-\frac{b}{4} \bar{\Xi}_{11}^{0}\left(p_{2}\right) I \\
i \frac{d}{d t} \Sigma_{22}^{\Phi \Phi}=\bar{g}_{0}\left(-p_{2}\right) \Sigma_{21}^{\Phi \Phi}+\bar{g}_{0}\left(p_{1}\right) \Sigma_{12}^{\Phi \Phi}
\end{gathered}
$$

where $I$ is the following integral :

$$
I=\int_{\mathbf{p}_{4}+\mathbf{p}_{5}=\mathbf{p}_{1}+\mathbf{p}_{2}} \frac{d^{3} p_{4}}{(2 \pi)^{3}} \frac{d^{3} p_{5}}{(2 \pi)^{3}} \Sigma_{11}^{\Phi \Phi}\left(\mathbf{p}_{4}, \mathbf{p}_{5}, \mathbf{p}_{3},-\sum \mathbf{p}_{i}, t, t^{\prime \prime}\right)
$$

These equations are analogous to (2.80), the matrices $\mathcal{H}_{0}, r$ and $R$ being replaced by the time-independent matrices $\overline{\mathcal{H}}_{0}, \bar{r}$ and $\bar{R}$ associated to $\bar{H}$ and $\Xi^{(0)}$ by $\bar{\Xi}_{0}$. We see that we are not abble to obtain directly an expression analogous to (3.41) which would relate the matrices $\Sigma\left(t_{0}, t^{\prime \prime}\right)$ and $\Sigma\left(t_{0}+i \beta, t^{\prime \prime}\right)$ since the loop term $\frac{b}{4} \bar{\Xi}_{11}^{0} I$ involve all the momenta. We will use a perturbative expansion where we neglect this loop term to lowest order. From the expression for $\bar{\Xi}_{11}^{0}$, we see that this expansion will be valid when

$$
\frac{b}{4} \operatorname{coth} \frac{\beta m(\beta)}{2} \ll 1
$$

where $m(\beta)$ is the self-consistent mass defined by :

$$
m^{2}(\beta)=m_{0}^{2}+\frac{b}{2} \int \frac{d^{3} p}{(2 \pi)^{3}} \frac{1}{2 \sqrt{\mathbf{p}^{2}+m^{2}(\beta)}} \operatorname{coth}\left(\frac{\beta}{2} \sqrt{\mathbf{p}^{2}+m^{2}(\beta)}\right)
$$

At zero temperature, the condition (3.94) for the validity of the perturbative expansion writes simply $\frac{b}{4} \ll 1$. At high temperature it becomes : $\frac{b}{2} \ll \beta m(\beta)$ with $m^{2}(\beta) \simeq$ $\bar{m}^{2}+\frac{b}{24 \beta^{2}}$.

To lowest order, by neglecting the loop term I, the solutions of equations (3.89)(3.92) write :

$$
\begin{gathered}
\Sigma_{11}^{\Phi \Phi}\left(\mathbf{k},-\mathbf{q}-\mathbf{k}, \mathbf{p}_{1}, \mathbf{q}-\mathbf{p}_{\mathbf{1}}, u, t^{\prime \prime}\right)=A_{1} \cosh \left(\Omega_{3} u\right)+B_{1} \sinh \left(\Omega_{3} u\right)+C_{1} \cosh \left(\Omega_{4} u\right)+D_{1} \sinh \left(\Omega_{4} u\right) \\
\Sigma_{12}^{\Phi \Phi}\left(u, t^{\prime \prime}\right)=\omega_{\mathbf{q}+\mathbf{k}}\left[A_{1} \sinh \left(\Omega_{3} u\right)+B_{1} \cosh \left(\Omega_{3} u\right)+C_{1} \sinh \left(\Omega_{4} u\right)+D_{1} \cosh \left(\Omega_{4} u\right)\right] \\
\Sigma_{21}^{\Phi}\left(u, t^{\prime \prime}\right)=\omega_{\mathbf{k}}\left[A_{1} \sinh \left(\Omega_{3} u\right)+B_{1} \cosh \left(\Omega_{3} u\right)-C_{1} \sinh \left(\Omega_{4} u\right)-D_{1} \cosh \left(\Omega_{4} u\right)\right]
\end{gathered}
$$




$$
\Sigma_{22}^{\Phi \Phi}\left(u, t^{\prime \prime}\right)=\omega_{\mathbf{k}} \omega_{\mathbf{q}+\mathbf{k}}\left[A_{1} \cosh \left(\Omega_{3} u\right)+B_{1} \sinh \left(\Omega_{3} u\right)-C_{1} \cosh \left(\Omega_{4} u\right)-D_{1} \sinh \left(\Omega_{4} u\right)\right]
$$

where we have used the variable $u$ defined by $: t=t_{0}+i(\beta-u)$ and we have introduced the frequencies

$$
\begin{aligned}
& \Omega_{3}=\omega_{\mathbf{q}+\mathbf{k}}+\omega_{\mathbf{k}} \\
& \Omega_{4}=\omega_{\mathbf{q}+\mathbf{k}}-\omega_{\mathbf{k}}
\end{aligned}
$$

$\omega_{\mathbf{k}}$ being defined by $\omega_{\mathbf{k}}=\sqrt{-\bar{g}_{0}(\mathbf{k})}$.

We can therefore express $\Sigma^{\Phi \Phi}\left(\mathbf{k},-\mathbf{q}-\mathbf{k}, \mathbf{p}_{1}, \mathbf{q}-\mathbf{p}_{1}, t_{0}+i \beta, t^{\prime \prime}\right)$ as a function of $\Sigma^{\Phi \Phi}\left(\mathbf{k},-\mathbf{q}-\mathbf{k}, \mathbf{p}_{1}, \mathbf{q}-\mathbf{p}_{1}, t_{0}, t^{\prime \prime}\right)$. The result is the following :

$\Sigma^{\Phi \Phi}\left(\mathbf{k},-\mathbf{q}-\mathbf{k}, \mathbf{p}_{1}, \mathbf{q}-\mathbf{p}_{1}, t_{0}+i \beta, t^{\prime \prime}\right)=A^{-1}(\mathbf{k}) \Sigma^{\Phi \Phi}\left(\mathbf{k},-\mathbf{q}-\mathbf{k}, \mathbf{p}_{1}, \mathbf{q}-\mathbf{p}_{1}, t_{0}, t^{\prime \prime}\right)\left(A^{T}\right)^{-1}(\mathbf{q}+\mathbf{k})$

where the matrix $A(\mathbf{k})$ is defined by (3.42) :

$$
\begin{gathered}
A(\mathbf{k})=\left(\begin{array}{cc}
\cosh \left(\omega_{\mathbf{k}} \beta\right) & \frac{\sinh \left(\omega_{\mathbf{k}} \beta\right)}{\omega_{\mathbf{k}}} \\
\omega_{\mathbf{k}} \sinh \left(\omega_{\mathbf{k}} \beta\right) & \cosh \left(\omega_{\mathbf{k}} \beta\right)
\end{array}\right) \\
A^{-1}(\mathbf{k})=\left(\begin{array}{cc}
\cosh \left(\omega_{\mathbf{k}} \beta\right) & -\frac{\sinh \left(\omega_{\mathbf{k}} \beta\right)}{\omega_{\mathbf{k}}} \\
-\omega_{\mathbf{k}} \sinh \left(\omega_{\mathbf{k}} \beta\right) & \cosh \left(\omega_{\mathbf{k}} \beta\right)
\end{array}\right)
\end{gathered}
$$

By introducing a column formed with the elements $\Sigma_{i j}$, we can write this expression in the following way, which will be usefull in section 3.6.4 :

$$
\left(\begin{array}{c}
\Sigma_{11}^{\Phi \Phi} \\
\Sigma_{12}^{\Phi \Phi} \\
\Sigma_{21}^{\Phi \Phi} \\
\Sigma_{22}^{\Phi \Phi}
\end{array}\right)\left(\mathbf{k},-\mathbf{q}-\mathbf{k}, \mathbf{p}_{1}, \mathbf{q}-\mathbf{p}_{1}, t_{0}, t^{\prime \prime}\right)=N(\mathbf{k}, \mathbf{q}+\mathbf{k})\left(\begin{array}{c}
\Sigma_{11}^{\Phi \Phi} \\
\Sigma_{12}^{\Phi \Phi} \\
\Sigma_{21}^{\Phi \Phi} \\
\Sigma_{22}^{\Phi \Phi}
\end{array}\right)\left(\mathbf{k},-\mathbf{q}-\mathbf{k}, \mathbf{p}_{1}, \mathbf{q}-\mathbf{p}_{1}, t_{0}+i \beta, t^{\prime \prime}\right)
$$

where $N(\mathbf{k}, \mathbf{q}+\mathbf{k})$ is the following 4 by 4 matrice :

$$
N(\mathbf{k}, \mathbf{q}+\mathbf{k})=\left(\begin{array}{cccc}
A & \frac{C}{\omega_{\mathbf{k}+\mathbf{q}}} & \frac{D}{\omega_{\mathbf{k}}} & \frac{B}{\omega_{\mathbf{k}} \omega_{\mathbf{k}+\mathbf{q}}} \\
\omega_{\mathbf{k}+\mathbf{q}} C & A & \frac{\omega_{\mathbf{k}+\mathbf{q}}}{\omega_{\mathbf{k}}} B & \frac{D}{\omega_{\mathbf{k}}} \\
\omega_{k} D & \frac{\omega_{k}}{\omega_{\mathbf{k}+\mathbf{q}}} B & A & \frac{C}{\omega_{\mathbf{k}+\mathbf{q}}} \\
\omega_{\mathbf{k}} \omega_{\mathbf{k}+\mathbf{q}} B & \omega_{\mathbf{k}} D & \omega_{\mathbf{k}+\mathbf{q}} C & A
\end{array}\right)
$$

with

$$
\begin{aligned}
& A=\cosh \left(\omega_{\mathbf{k}+\mathbf{q}} \beta\right) \cosh \left(\omega_{\mathbf{k}} \beta\right) \\
& B=\sinh \left(\omega_{\mathbf{k}+\mathbf{q}} \beta\right) \sinh \left(\omega_{\mathbf{k}} \beta\right) \\
& C=\sinh \left(\omega_{\mathbf{k}+\mathbf{q}} \beta\right) \cosh \left(\omega_{\mathbf{k}} \beta\right) \\
& D=\cosh \left(\omega_{\mathbf{k}+\mathbf{q}} \beta\right) \sinh \left(\omega_{\mathbf{k}} \beta\right)
\end{aligned}
$$


If we do not optimize with respect to the initial state, an approximation for the four-point function $\Sigma_{11}^{\Phi \Phi}\left(t^{\prime}, t^{\prime \prime}\right)$ is given by equation (2.111) for $t^{\prime \prime}>t^{\prime}$.

On the other hand, if we optimize with respect to the initial state, $\Sigma_{11}^{\Phi \Phi}\left(t^{\prime}, t^{\prime \prime}\right)$ is given by (2.110) for $t^{\prime \prime}>t^{\prime}$ :

$$
\begin{aligned}
& \Sigma_{11}^{\Phi \Phi}\left(\mathbf{p}_{1}, \mathbf{p}_{2}, \mathbf{p}_{3},-\sum \mathbf{p}_{i}, t^{\prime}, t^{\prime \prime}\right)=-\int \frac{d^{3} p}{(2 \pi)^{3}} \times \\
& \operatorname{tr}\left[l^{\Phi \Phi}\left(\mathbf{p}-\mathbf{p}_{1}-\mathbf{p}_{2},-\mathbf{p}, \mathbf{p}_{1}, \mathbf{p}_{2}, t^{\prime}, t_{0}\right) \Sigma^{\Phi \Phi}\left(\mathbf{p},-\mathbf{p}+\mathbf{p}_{1}+\mathbf{p}_{2}, \mathbf{p}_{3},-\sum \mathbf{p}_{i}, t_{0}, t^{\prime \prime}\right)\right]
\end{aligned}
$$

and we use (3.51) and (3.53) to write

$$
\begin{aligned}
& \Sigma^{\Phi \Phi}\left(\mathbf{p}_{1}, \mathbf{p}_{2}, \mathbf{p}_{3},-\sum \mathbf{p}_{i}, t_{0}+i \beta, t^{\prime \prime}\right)=\Sigma^{\Phi \Phi}\left(\mathbf{p}_{1}, \mathbf{p}_{2}, \mathbf{p}_{3},-\sum \mathbf{p}_{i}, t_{0}, t^{\prime \prime}\right) \\
& +2\left[\bar{\Xi}_{0}\left(-\mathbf{p}_{1}\right) l^{\Phi \Phi}\left(\mathbf{p}_{1}, \mathbf{p}_{2}, \mathbf{p}_{3},-\sum \mathbf{p}_{i}, t^{\prime \prime}, t_{0}\right) \tau-\tau l^{\Phi \Phi}\left(\mathbf{p}_{1}, \mathbf{p}_{2}, \mathbf{p}_{3},-\sum \mathbf{p}_{i}, t^{\prime \prime}, t_{0}\right) \bar{\Xi}_{0}\left(\mathbf{p}_{2}\right)\right]
\end{aligned}
$$

or :

$$
\begin{aligned}
& A^{-1}(\mathbf{k}) \Sigma^{\Phi \Phi}\left(\mathbf{k},-\mathbf{q}-\mathbf{k}, \mathbf{p}_{1}, \mathbf{q}-\mathbf{p}_{1}, t_{0}, t^{\prime \prime}\right)\left(A^{T}\right)^{-1}(\mathbf{q}+\mathbf{k})-\Sigma^{\Phi \Phi}\left(\mathbf{k},-\mathbf{q}-\mathbf{k}, \mathbf{p}_{1}, \mathbf{q}-\mathbf{p}_{1}, t_{0}, t^{\prime \prime}\right)= \\
& 2\left[\bar{\Xi}_{0}(-\mathbf{k}) l^{\Phi \Phi}\left(\mathbf{k},-\mathbf{q}-\mathbf{k}, \mathbf{p}_{1}, \mathbf{q}-\mathbf{p}_{1}, t^{\prime \prime}, t_{0}\right) \tau-\tau l^{\Phi \Phi}\left(\mathbf{k},-\mathbf{q}-\mathbf{k}, \mathbf{p}_{1}, \mathbf{q}-\mathbf{p}_{1}, t^{\prime \prime}, t_{0}\right) \bar{\Xi}_{0}(-\mathbf{q}-\mathbf{k})\right]
\end{aligned}
$$

where $\bar{\Xi}_{0}$ is given by $(2.57), g_{0}$ being replaced by $\bar{g}_{0}$.

The matrix elements $\Sigma_{i j}^{\Phi \Phi}\left(t_{0}, t^{\prime \prime}\right)$ can therefore be written in terms of $l_{i j}^{\Phi \Phi}\left(t^{\prime \prime}, t_{0}\right)$. By using (3.111), we finally obtain an approximation for the two-time and four-point function $\Sigma_{11}^{\Phi \Phi}\left(t^{\prime}, t^{\prime \prime}\right)$ which involves only the function $l^{\Phi \Phi}$. We recall that this approximation has been obtained by neglecting the loop term I (3.93) in the evolution equations of $\Sigma^{\Phi \Phi}$ for complex values of the time.

\subsubsection{Resolution of the dynamical equations for $l^{\Phi \Phi}$ in the static case}

The dynamical equations for the matrix elements of $l^{\Phi \Phi}$ are given by (2.89). In the static case, $\Xi^{(0)}(t)$ is equal to $\Xi_{0}$ given by (2.57), $\alpha^{(0)}(t)=\alpha_{0}$ and $g_{0}$ is time-independent. In the symmetric phase, equations (2.89) write more explicitly in momentum space :

$$
\begin{gathered}
i \frac{d}{d t} l_{11}^{\Phi \Phi}\left(\mathbf{k},-\mathbf{q}-\mathbf{k}, \mathbf{p}_{i},-\mathbf{p}_{i}+\mathbf{q}, t^{\prime \prime}, t\right)=-g_{0}(\mathbf{k}) l_{21}^{\Phi \Phi}-g_{0}(\mathbf{q}+\mathbf{k}) l_{12}^{\Phi \Phi}+J \\
i \frac{d}{d t} l_{12}^{\Phi \Phi}=l_{11}^{\Phi \Phi}-g_{0}(\mathbf{k}) l_{22}^{\Phi \Phi} \\
i \frac{d}{d t} l_{21}^{\Phi \Phi}=l_{11}^{\Phi \Phi}-g_{0}(\mathbf{q}+\mathbf{k}) l_{22}^{\Phi \Phi} \\
i \frac{d}{d t} l_{22}^{\Phi \Phi}=l_{12}^{\Phi \Phi}+l_{21}^{\Phi \Phi}
\end{gathered}
$$


with the boundary conditions (2.127). $\mathrm{J}$ is the following integral :

$$
\begin{gathered}
J=-\frac{b}{4} \int \frac{d^{3} p}{(2 \pi)^{3}} \quad\left[\Xi_{0}(p) l^{\Phi \Phi}\left(-\mathbf{p},-\mathbf{q}+\mathbf{p}, \mathbf{p}_{i},-\mathbf{p}_{i}+\mathbf{q}, t^{\prime \prime}, t\right) \tau\right. \\
\left.-\tau l^{\Phi \Phi}\left(-\mathbf{q}-\mathbf{p}, \mathbf{p}, \mathbf{p}_{i},-\mathbf{p}_{i}+\mathbf{q}, t^{\prime \prime}, t\right) \Xi_{0}(p)\right]_{11} \\
J=\frac{b}{4} \int \frac{d^{3} p}{(2 \pi)^{3}} \frac{1}{\omega_{p}} \operatorname{coth}\left(\frac{\beta \omega_{p}}{2}\right)\left[l_{12}^{\Phi \Phi}\left(-\mathbf{p},-\mathbf{q}+\mathbf{p}, \mathbf{p}_{i},-\mathbf{p}_{i}+\mathbf{q}, t^{\prime \prime}, t\right)+l_{21}^{\Phi \Phi}\left(-\mathbf{q}-\mathbf{p}, \mathbf{p}, \mathbf{p}_{i},-\mathbf{p}_{i}+\mathbf{q}, t^{\prime \prime}, t\right)\right]
\end{gathered}
$$

In the previous equations, in order to make the notations more compact, we have ommitted when it was possible the arguments of $l^{\Phi \Phi}$. Let us remark that, on the contrary to equations (3.89)-(3.92) for $\Sigma^{\Phi \Phi}$, the solution $\Xi_{0}(p)$ appears inside the loop integral.

Similarly to the resolution of the equations for $\Sigma^{\Phi \Phi}$, we will solve the equations for $l^{\Phi \Phi}$, perturbatively, neglecting first the loop term J. In the following section, we will take into account of the loop term $\mathrm{J}$ to first order. To lowest order, the solutions of (3.114)-(3.117) write :

$l_{11}^{\Phi \Phi}\left(\mathbf{k},-\mathbf{q}-\mathbf{k}, \mathbf{p}_{1}, \mathbf{q}-\mathbf{p}_{1}, t^{\prime}, t\right)=-\frac{1}{2}\left[\delta^{3}\left(\mathbf{k}+\mathbf{p}_{1}\right)+\delta^{3}\left(-\mathbf{q}-\mathbf{k}+\mathbf{p}_{1}\right)\right] \cos \left(\omega_{\mathbf{k}+\mathbf{q}}\left(t-t^{\prime}\right)\right) \cos \left(\omega_{\mathbf{k}}\left(t-t^{\prime}\right)\right)$

$l_{12}^{\Phi \Phi}\left(\mathbf{k},-\mathbf{q}-\mathbf{k}, \mathbf{p}_{1}, \mathbf{q}-\mathbf{p}_{1}, t^{\prime}, t\right)=-\frac{1}{2 i \omega_{\mathbf{k}+\mathbf{q}}}\left[\delta^{3}\left(\mathbf{k}+\mathbf{p}_{1}\right)+\delta^{3}\left(-\mathbf{q}-\mathbf{k}+\mathbf{p}_{1}\right)\right] \sin \left(\omega_{\mathbf{k}+\mathbf{q}}\left(t-t^{\prime}\right)\right) \cos \left(\omega_{\mathbf{k}}\left(t-t^{\prime}\right)\right)$

$l_{21}^{\Phi \Phi}\left(\mathbf{k},-\mathbf{q}-\mathbf{k}, \mathbf{p}_{1}, \mathbf{q}-\mathbf{p}_{1}, t^{\prime}, t\right)=-\frac{1}{2 i \omega_{\mathbf{k}}}\left[\delta^{3}\left(\mathbf{k}+\mathbf{p}_{1}\right)+\delta^{3}\left(-\mathbf{q}-\mathbf{k}+\mathbf{p}_{1}\right)\right] \cos \left(\omega_{\mathbf{k}+\mathbf{q}}\left(t-t^{\prime}\right)\right) \sin \left(\omega_{\mathbf{k}}\left(t-t^{\prime}\right)\right)$

$l_{22}^{\Phi \Phi}\left(\mathbf{k},-\mathbf{q}-\mathbf{k}, \mathbf{p}_{1}, \mathbf{q}-\mathbf{p}_{1}, t^{\prime}, t\right)=\frac{1}{2 \omega_{\mathbf{k}} \omega_{\mathbf{k}+\mathbf{q}}}\left[\delta^{3}\left(\mathbf{k}+\mathbf{p}_{1}\right)+\delta^{3}\left(-\mathbf{q}-\mathbf{k}+\mathbf{p}_{1}\right)\right] \sin \left(\omega_{\mathbf{k}+\mathbf{q}}\left(t-t^{\prime}\right)\right) \sin \left(\omega_{\mathbf{k}}\left(t-t^{\prime}\right)\right)$

Formula (3.111) and (3.113) then give, for $\Delta t=t^{\prime}-t^{\prime \prime}<0$ :

$\Sigma_{11}^{\Phi \Phi}\left(\mathbf{k},-\mathbf{q}-\mathbf{k}, \mathbf{p}_{1}, \mathbf{q}-\mathbf{p}_{1}, t^{\prime}, t^{\prime \prime}\right)=2\left[\delta^{3}\left(\mathbf{k}+\mathbf{p}_{1}\right)+\delta^{3}\left(-\mathbf{q}-\mathbf{k}+\mathbf{p}_{1}\right)\right] \beta_{1}^{\Phi}(\mathbf{k}, \Delta t) \beta_{1}^{\Phi}(\mathbf{q}+\mathbf{k}, \Delta t)$

where $\beta_{1}^{\Phi}(\mathbf{k}, \Delta t)$ is given by (3.66). We thus have at the lowest order :

$$
\Pi^{(0)}\left(\mathbf{q}^{2}, \Delta t\right)=2 i \int \frac{d^{3} k}{(2 \pi)^{3}} \beta_{1}^{\Phi}(\mathbf{k}, \Delta t) \beta_{1}^{\Phi}(\mathbf{q}+\mathbf{k}, \Delta t)
$$

When we neglect the loop terms $I$ and $J$ in the dynamical equations for $\Sigma^{\Phi \Phi}$ for the complex time and in the dynamical equations for $l^{\Phi \Phi}$ for the time $t>t_{0}$, we thus recover the Wick theorem as we should. Let us stress that we have neglected the interactions which appear in the terms I and J but the interactions remain in the frequencies $\omega_{k}$ which involve the renormalized self-consistent mass $m(\beta)$ (eq. (2.66)).

At this order, $\Sigma_{11}^{\Phi \Phi}$ depends only on the time difference $\Delta t=t^{\prime}-t^{\prime \prime}$ and our approximation satisfies the fluctuation-dissipation theorem for the two-time and four-point correlation function. 
If we apply formula (2.111), obtained without optimization with respect to the initial state, with the solutions $l^{\Phi \Phi}$ given by (3.120)-(3.123), we find again the result of the Wick theorem. When we neglect the terms with the interaction in the dynamical equations for $\Sigma^{\Phi \Phi}$ and $l^{\Phi \Phi}$, the two approximations for $\Sigma_{11}^{\Phi \Phi}\left(t^{\prime}, t^{\prime \prime}\right)$ obtained with or without optimization with respect to the initial state therefore coïncide. Accordingly, at zero order, we can use instead of expression (3.113) the simpler expression :

$\Sigma^{\Phi \Phi}\left(\mathbf{k},-\mathbf{q}-\mathbf{k}, \mathbf{p}_{1},-\mathbf{p}_{1}+\mathbf{q}, t_{0}, t^{\prime \prime}\right)=-\left(\bar{\Xi}_{0}(\mathbf{k})-\tau\right) l^{\Phi \Phi}\left(\mathbf{k},-\mathbf{q}-\mathbf{k}, \mathbf{p}_{1}, \mathbf{q}-\mathbf{p}_{1}, t^{\prime \prime}, t_{0}\right)\left(\bar{\Xi}_{0}(-\mathbf{q}-\mathbf{k})+\tau\right)$

The existence of such an expression with matrices (to distinguish from a vectorial expression like (3.105) was not obvious at the beginning.

For the polarization function $\Pi_{R}\left(\mathbf{q}^{2}, t^{\prime}, t^{\prime \prime}\right)$, we obtain to lowest order :

$$
\begin{aligned}
\Pi_{R}^{(0)}\left(\mathbf{q}^{2}, t^{\prime}, t^{\prime \prime}\right)= & -\theta\left(t^{\prime}-t^{\prime \prime}\right) \int \frac{d^{3} p}{(2 \pi)^{3}} \frac{1}{\omega_{\mathbf{p}} \omega_{\mathbf{q}+\mathbf{p}}} \\
& {\left[\operatorname{coth} \frac{\beta \omega_{\mathbf{p}}}{2} \cos \omega_{\mathbf{p}} \Delta t \sin \omega_{\mathbf{p}+\mathbf{q}} \Delta t+\operatorname{coth} \frac{\beta \omega_{\mathbf{p}+\mathbf{q}}}{2} \cos \omega_{\mathbf{p}+\mathbf{q}} \Delta t \sin \omega_{\mathbf{p}} \Delta t\right] }
\end{aligned}
$$

Its Fourrier transform in time writes :

$$
\begin{aligned}
& \Pi_{R}^{(0)} \quad\left(\mathbf{q}^{2}, \omega\right)=\int \frac{d^{3} p}{(2 \pi)^{3}} \frac{1}{\omega_{\mathbf{p}} \omega_{\mathbf{q}+\mathbf{p}}} \\
& {\left[\left(n_{\mathbf{p}+\mathbf{q}}+n_{\mathbf{p}}+1\right) \frac{\omega_{\mathbf{p}+\mathbf{q}}+\omega_{\mathbf{p}}}{(\omega+i \eta)^{2}-\left(\omega_{\mathbf{p}+\mathbf{q}}+\omega_{\mathbf{p}}\right)^{2}}-\left(n_{\mathbf{p}+\mathbf{q}}-n_{\mathbf{p}}\right) \frac{\omega_{\mathbf{p}+\mathbf{q}}-\omega_{\mathbf{p}}}{(\omega+i \eta)^{2}-\left(\omega_{\mathbf{p}+\mathbf{q}}-\omega_{\mathbf{p}}\right)^{2}}\right] }
\end{aligned}
$$

A similar expression appears for instance in [22. Here, we have derived it variationally. We stress again that this formula is nonperturbative in the sense that it contains the renormalized self-consistent mass.

\subsubsection{Resolution of the dynamical equations with the interaction terms I and $\mathrm{J}$ at first order}

At first order, we will solve the dynamical equations (3.89)-3.92) for

$\Sigma^{\Phi \Phi}\left(\mathbf{k},-\mathbf{q}-\mathbf{k}, \mathbf{p}_{1},-\mathbf{p}_{1}+\mathbf{q}, u, t^{\prime \prime}\right)$ by replacing in the loop integral I the solution $\Sigma^{\Phi \Phi}$ obtained at the lowest order. We thus have to solve a first order differential sytem with a nonhomogeneous term which involves :

$$
I(u)=\int \frac{d^{3} l}{(2 \pi)^{3}}\left[a \cosh \left(\Omega_{5} u\right)+b \sinh \left(\Omega_{5} u\right)+c \cosh \left(\Omega_{6} u\right)+d \sinh \left(\Omega_{6} u\right)\right]
$$

with (see the analogous definitions (3.100) and (3.101) ) :

$$
\Omega_{5}=\omega_{\mathbf{q}+\mathbf{l}}+\omega_{\mathbf{l}}
$$




$$
\Omega_{6}=\omega_{\mathbf{q}+\mathbf{l}}-\omega_{\mathbf{l}}
$$

The constants a,b,c and d depend on the momenta $\mathbf{l}$ and $\mathbf{q}$. They can be expressed in terms of the matrix elements $\Sigma_{i j}\left(\mathbf{l}, \mathbf{- q}-\mathbf{l}, \mathbf{p}_{i}, \mathbf{q}-\mathbf{p}_{i}, t_{0}, t^{\prime \prime}\right)$ and $\cosh \left(\Omega_{5} \beta\right), \sinh \left(\Omega_{6} \beta\right), \cosh \left(\Omega_{6} \beta\right)$, $\sinh \left(\Omega_{6} \beta\right)$. At the order we consider, we can use (3.126) and express $a, b, c, d$ with the matrix elements $l_{i j}^{\Phi \Phi}\left(\mathbf{l},-\mathbf{q}-\mathbf{l}, \mathbf{p}_{i}, \mathbf{q}-\mathbf{p}_{i}, t^{\prime \prime}, t_{0}\right)$, with $\operatorname{coth}\left(\frac{\beta \Omega_{5}}{2}\right), \operatorname{coth}\left(\frac{\beta \Omega_{6}}{2}\right)$ and with the occupation numbers $n_{\mathbf{l}}$ et $n_{\mathbf{l}+\mathbf{q}}$ defined by :

$$
2 n_{\mathbf{l}}+1=\operatorname{coth}\left(\frac{\beta \omega_{\mathbf{l}}}{2}\right)
$$

The relation between $\Sigma_{i j}^{\Phi \Phi}\left(t_{0}, t^{\prime \prime}\right)$ and $\Sigma_{i j}^{\Phi \Phi}\left(t_{0}+i \beta, t^{\prime \prime}\right)$ writes now :

$$
\left(\begin{array}{c}
\Sigma_{11}^{\Phi \Phi} \\
\Sigma_{12}^{\Phi \Phi} \\
\Sigma_{21}^{\Phi \Phi} \\
\Sigma_{22}^{\Phi \Phi}
\end{array}\right)\left(\mathbf{k},-\mathbf{q}-\mathbf{k}, \mathbf{p}_{1}, \mathbf{q}-\mathbf{p}_{1}, t_{0}, t^{\prime \prime}\right)=N(\mathbf{k}, \mathbf{q}+\mathbf{k})\left(\begin{array}{c}
\Sigma_{11}^{\Phi \Phi} \\
\Sigma_{12}^{\Phi \Phi} \\
\Sigma_{21}^{\Phi \Phi} \\
\Sigma_{22}^{\Phi \Phi}
\end{array}\right)\left(\mathbf{k},-\mathbf{q}-\mathbf{k}, \mathbf{p}_{1}, \mathbf{q}-\mathbf{p}_{1}, t_{0}+i \beta, t^{\prime \prime}\right)+W\left(t_{0}, t^{\prime \prime}\right)
$$

where the matrix $N(\mathbf{k}, \mathbf{q}+\mathbf{k})$ given by (3.106) does not contain explicitly the coupling constant and $\mathrm{W}$ is a vector whose components are proportional to the coupling constant $b$.

By using the fact that the first term can be written as (3.126), we write in matrix notation :

$$
\begin{aligned}
\Sigma^{\Phi \Phi}\left(\mathbf{k},-\mathbf{q}-\mathbf{k}, \mathbf{p}_{1}, \mathbf{q}-\mathbf{p}_{1}, t_{0}, t^{\prime \prime}\right)= & -\left(\bar{\Xi}_{0}(\mathbf{k})-\tau\right) l^{\Phi \Phi}\left(\mathbf{k},-\mathbf{q}-\mathbf{k}, \mathbf{p}_{1}, \mathbf{q}-\mathbf{p}_{1}, t^{\prime \prime}, t_{0}\right) \\
& \times\left(\bar{\Xi}_{0}(-\mathbf{q}-\mathbf{k})+\tau\right)+X\left(t_{0}, t^{\prime \prime}\right)
\end{aligned}
$$

where

$$
X\left(t_{0}, t^{\prime \prime}\right)=\left(\begin{array}{ll}
X_{1} & X_{2} \\
X_{3} & X_{4}
\end{array}\right)
$$

and

$$
X_{i}=(1-N)_{i j}^{-1} W_{j}
$$

$X\left(t_{0}, t^{\prime \prime}\right)$ depends on the momenta $\mathbf{k}, \mathbf{k}+\mathbf{q}$. The elements $X_{i}$ can be expressed relatively simply with the integrals $I_{n}, J_{n}, K_{n}, L_{n}, M_{n}$ of Appendix E :

$$
\begin{aligned}
& X_{1}=\frac{b}{8 \omega_{\mathbf{k}} \omega_{\mathbf{k}+\mathbf{q}}}\left\{\quad ( n _ { \mathbf { k } + \mathbf { q } } + n _ { \mathbf { k } } + 1 ) \left[-\Omega_{3}\left(I_{1}+I_{2}+K_{1}+K_{2}+K_{3}+K_{4}\right)\right.\right. \\
& \left.-\operatorname{coth}\left(\frac{\beta \Omega_{3}}{2}\right)\left(J_{1}+J_{2}-M_{1}-M_{2}-M_{3}-M_{4}\right)\right] \\
& \left(n_{\mathbf{k}}-n_{\mathbf{k}+\mathbf{q}}\right)\left[-\Omega_{4}\left(I_{3}+I_{4}+L_{1}+L_{2}+L_{3}+L_{4}\right)\right. \\
& \left.-\operatorname{coth}\left(\frac{\beta \Omega_{4}}{2}\right)\left(J_{4}+J_{3}-N_{1}-N_{2}-N_{3}-N_{4}\right)\right]
\end{aligned}
$$




$$
\begin{aligned}
& X_{2}=\frac{b}{8 \omega_{\mathbf{k}}}\left\{\quad ( n _ { \mathbf { k } + \mathbf { q } } + n _ { \mathbf { k } } + 1 ) \left[-\Omega_{3} \operatorname{coth}\left(\frac{\beta \Omega_{3}}{2}\right)\left(I_{1}+I_{2}-K_{1}-K_{2}-K_{3}-K_{4}\right)\right.\right. \\
& \left.-\left(J_{1}+J_{2}+M_{1}+M_{2}+M_{3}+M_{4}\right)\right] \\
& \left(n_{\mathbf{k}}-n_{\mathbf{k}+\mathbf{q}}\right)\left[-\Omega_{4} \operatorname{coth}\left(\frac{\beta \Omega_{4}}{2}\right)\left(I_{3}+I_{4}-L_{1}-L_{2}-L_{3}-L_{4}\right)\right. \\
& \left.-\left(J_{4}+J_{3}+N_{1}+N_{2}+N_{3}+N_{4}\right)\right] \\
& X_{3}=\frac{b}{8 \omega_{\mathbf{k}+\mathbf{q}}}\left\{( n _ { \mathbf { k } + \mathbf { q } } + n _ { \mathbf { k } } + 1 ) \left[-\Omega_{3} \operatorname{coth}\left(\frac{\beta \Omega_{3}}{2}\right)\left(I_{1}+I_{2}-K_{1}-K_{2}-K_{3}-K_{4}\right)\right.\right. \\
& \left.-\left(J_{1}+J_{2}+M_{1}+M_{2}+M_{3}+M_{4}\right)\right] \\
& -\left(n_{\mathbf{k}}-n_{\mathbf{k}+\mathbf{q}}\right)\left[-\Omega_{4} \operatorname{coth}\left(\frac{\beta \Omega_{4}}{2}\right)\left(I_{3}+I_{4}-L_{1}-L_{2}-L_{3}-L_{4}\right)\right. \\
& \left.-\left(J_{4}+J_{3}+N_{1}+N_{2}+N_{3}+N_{4}\right)\right] \\
& X_{4}=\frac{b}{8}\left\{\quad ( n _ { \mathbf { k } + \mathbf { q } } + n _ { \mathbf { k } } + 1 ) \left[-\Omega_{3}\left(I_{1}+I_{2}+K_{1}+K_{2}+K_{3}+K_{4}\right)\right.\right. \\
& \left.-\operatorname{coth}\left(\frac{\beta \Omega_{3}}{2}\right)\left(J_{1}+J_{2}-M_{1}-M_{2}-M_{3}-M_{4}\right)\right] \\
& -\left(n_{\mathbf{k}}-n_{\mathbf{k}+\mathbf{q}}\right)\left[-\Omega_{4}\left(I_{3}+I_{4}+L_{1}+L_{2}+L_{3}+L_{4}\right)\right. \\
& \left.-\operatorname{coth}\left(\frac{\beta \Omega_{4}}{2}\right)\left(J_{4}+J_{3}-N_{1}-N_{2}-N_{3}-N_{4}\right)\right]
\end{aligned}
$$

By using the expressions of Appendix E, we obtain the elements $X_{i}$ in terms of $l_{i j}^{\Phi \Phi}$.

The approximation for the function $\Pi\left(\mathbf{q}^{2}, t^{\prime}, t^{\prime \prime}\right)$ is given by :

$$
\Pi\left(\mathbf{q}^{2}, t^{\prime}, t^{\prime \prime}\right)=\frac{i}{2} \int \frac{d^{3} k}{(2 \pi)^{3}} \frac{d^{3} p_{1}}{(2 \pi)^{3}} \Sigma_{11}^{\Phi \Phi}\left(\mathbf{k}, \mathbf{q}-\mathbf{k}, \mathbf{p}_{1}, \mathbf{q}-\mathbf{p}_{1}, t^{\prime}, t^{\prime \prime}\right)
$$

or

$$
\begin{aligned}
\Pi\left(\mathbf{q}^{2}, t^{\prime}, t^{\prime \prime}\right)=-\frac{i}{2} \int & \frac{d^{3} k}{(2 \pi)^{3}} \frac{d^{3} p_{1}}{(2 \pi)^{3}} \frac{d^{3} p}{(2 \pi)^{3}} \times \\
& \operatorname{tr}\left[l^{\Phi \Phi}\left(\mathbf{p}+\mathbf{q},-\mathbf{p}, \mathbf{k},-\mathbf{q}-\mathbf{k}, t^{\prime}, t_{0}\right) \Sigma^{\Phi \Phi}\left(\mathbf{p},-\mathbf{p}-\mathbf{q}, \mathbf{p}_{1}, \mathbf{q}-\mathbf{p}_{1}, t_{0}, t^{\prime \prime}\right)\right]
\end{aligned}
$$

It remains now to solve the dynamical equations for $l^{\Phi \Phi}$ taking into account of the loop term $J$ (3.118). We will do it in the static case. Equations (3.114)-(3.117) form a nonhomogeneous first order differential system. The solutions $l_{i j}^{\Phi \Phi}\left(\mathbf{k},-\mathbf{q}-\mathbf{k}, \mathbf{p}_{i},-\mathbf{p}_{i}+\right.$ $\left.\mathbf{q}, t^{\prime}, t^{\prime \prime}\right)$ are given in Appendix $\mathrm{F}$. The approximation to first order for $\Pi\left(\mathbf{q}^{2}, t^{\prime}, t^{\prime \prime}\right)$ is composed of two terms which are for $t^{\prime}<t^{\prime \prime}$ :

$$
\begin{aligned}
& \Pi\left(\mathbf{q}^{2}, t^{\prime}, t^{\prime \prime}\right)=\frac{i}{2} \int \frac{d^{3} k}{(2 \pi)^{3}} \frac{d^{3} p_{1}}{(2 \pi)^{3}} \frac{d^{3} p}{(2 \pi)^{3}} \times \\
& \operatorname{tr}\left[l^{\Phi \Phi}\left(\mathbf{p}+\mathbf{q},-\mathbf{p}, \mathbf{k},-\mathbf{q}-\mathbf{k}, t^{\prime}, t_{0}\right)\left(\bar{\Xi}_{0}(\mathbf{p})-\tau\right) l^{\Phi \Phi}\left(\mathbf{p},-\mathbf{p}-\mathbf{q}, \mathbf{p}_{1}, \mathbf{q}-\mathbf{p}_{1}, t^{\prime \prime}, t_{0}\right)\left(\bar{\Xi}_{0}(\mathbf{p}+\mathbf{q})+\tau\right)\right] \\
& -\operatorname{tr}\left[l^{\Phi \Phi}\left(\mathbf{p}+\mathbf{q},-\mathbf{p}, \mathbf{k},-\mathbf{q}-\mathbf{k}, t^{\prime}, t_{0}\right) X\left(t_{0}, t^{\prime \prime}\right)\right]
\end{aligned}
$$


The first term corresponds to the approximation one obtains without optimization with respect to the initial state (by replacing $\bar{\Xi}_{0}$ by $\Xi_{0}$ ). When one optimizes with respect to initial state, one has to take into account of the second term. We insert in the previous formula the expressions obtained to first order for the solutions $l^{\Phi \Phi}$ in the static case. We obtain the following result at the first order, for $\Delta t=t^{\prime}-t^{\prime \prime}<0$ :

$$
\begin{aligned}
\Pi^{(1)}\left(\mathbf{q}^{2}, t^{\prime}, t^{\prime \prime}\right)= & -\frac{i}{2} \frac{b}{2} \int \frac{d^{3} k}{(2 \pi)^{3}} \frac{d^{3} p_{1}}{(2 \pi)^{3}} \frac{1}{\omega_{\mathbf{k}} \omega_{\mathbf{k}+\mathbf{q}} \omega_{\mathbf{p}_{1}} \omega_{-\mathbf{p}_{1}+\mathbf{q}}} \times \\
& \left(n_{\mathbf{k}+\mathbf{q}}+n_{\mathbf{k}}+1\right)\left(\left(n_{-\mathbf{p}_{1}+\mathbf{q}}+n_{\mathbf{p}_{1}}+1\right) \frac{\Omega_{7}}{\Omega_{3}^{2}-\Omega_{7}^{2}}-\left(n_{-\mathbf{p}_{1}+\mathbf{q}}-n_{\mathbf{p}_{1}}\right) \frac{\Omega_{8}}{\Omega_{3}^{2}-\Omega_{8}^{2}}\right) \\
& \times\left(\operatorname{coth}\left(\frac{\beta \Omega_{3}}{2}\right) \cos \left(\Omega_{3} \Delta t\right)+i \sin \left(\Omega_{3} \Delta t\right)\right) \\
& -\left(n_{\mathbf{k}+\mathbf{q}}-n_{\mathbf{k}}\right)\left(\left(n_{-\mathbf{p}_{1}+\mathbf{q}}+n_{\mathbf{p}_{1}}+1\right) \frac{\Omega_{7}}{\Omega_{4}^{2}-\Omega_{7}^{2}}-\left(n_{-\mathbf{p}_{1}+\mathbf{q}}-n_{\mathbf{p}_{1}}\right) \frac{\Omega_{8}}{\Omega_{4}^{2}-\Omega_{8}^{2}}\right) \\
& \times\left(\operatorname{coth}\left(\frac{\beta \Omega_{4}}{2}\right) \cos \left(\Omega_{4} \Delta t\right)+i \sin \left(\Omega_{4} \Delta t\right)\right)
\end{aligned}
$$

with

$$
\begin{gathered}
\Omega_{3}=\omega_{\mathbf{k}+\mathbf{q}}+\omega_{\mathbf{k}} \quad, \quad \Omega_{4}=\omega_{\mathbf{k}+\mathbf{q}}-\omega_{\mathbf{k}} \\
\Omega_{7}=\omega_{-\mathbf{p}_{1}+\mathbf{q}}+\omega_{\mathbf{p}_{1}} \quad, \quad \Omega_{8}=\omega_{-\mathbf{p}_{1}+\mathbf{q}}-\omega_{-\mathbf{p}_{1}}
\end{gathered}
$$

This is the essential result of this section. One has to add to this formula the term (3.125) obtained to lowest order. In the static case, we obtain an approximation for the correlation function $\Pi\left(\mathbf{q}^{2}, t^{\prime}, t^{\prime \prime}\right)$ which depends only on the time difference $\Delta t=t^{\prime}-t^{\prime \prime}$. If we do not optimize with respect to the initial state, which corresponds to consider only the first term in (3.143), the approximation obtained for $\Pi\left(\mathbf{q}^{2}, t^{\prime}, t^{\prime \prime}\right)$ contains a spurious 
dependence on the initial time $t_{0}$ which writes :

$$
\begin{aligned}
& P^{(1)}=-\frac{i}{2} \int \frac{d^{3} k}{(2 \pi)^{3}} \frac{d^{3} p_{1}}{(2 \pi)^{3}} \frac{b}{4 \omega_{\mathbf{k}} \omega_{\mathbf{k}+\mathbf{q}} \omega_{\mathbf{p}_{1}} \omega_{-\mathbf{p}_{1}+\mathbf{q}}} \times \\
& +\frac{1}{2}\left(n_{\mathbf{k}+\mathbf{q}}+n_{\mathbf{k}}+1\right)\left(n_{-\mathbf{p}_{1}+\mathbf{q}}+n_{\mathbf{p}_{1}}+1\right) \frac{1}{\Omega_{3}^{2}-\Omega_{7}^{2}}\left(\Omega_{7} \operatorname{coth}\left(\frac{\beta \Omega_{3}}{2}\right)-\Omega_{3} \operatorname{coth}\left(\frac{\beta \Omega_{7}}{2}\right)\right) \\
& \times\left[\cos \Omega_{7}\left(t_{0}-t^{\prime \prime}\right) \cos \Omega_{3}\left(t_{0}-t^{\prime}\right)+\cos \Omega_{7}\left(t_{0}-t^{\prime}\right) \cos \Omega_{3}\left(t_{0}-t^{\prime \prime}\right)\right] \\
& +\frac{1}{2}\left(n_{\mathbf{k}+\mathbf{q}}+n_{\mathbf{k}}+1\right)\left(n_{-\mathbf{p}_{1}+\mathbf{q}}+n_{\mathbf{p}_{1}}+1\right) \frac{1}{\Omega_{3}^{2}-\Omega_{7}^{2}}\left(\Omega_{3} \operatorname{coth}\left(\frac{\beta \Omega_{3}}{2}\right)-\Omega_{7} \operatorname{coth}\left(\frac{\beta \Omega_{7}}{2}\right)\right) \\
& \times\left[\sin \Omega_{7}\left(t_{0}-t^{\prime \prime}\right) \sin \Omega_{3}\left(t_{0}-t^{\prime}\right)+\sin \Omega_{7}\left(t_{0}-t^{\prime}\right) \sin \Omega_{3}\left(t_{0}-t^{\prime \prime}\right)\right] \\
& -\frac{1}{2}\left(n_{\mathbf{k}+\mathbf{q}}-n_{\mathbf{k}}\right)\left(n_{-\mathbf{p}_{1}+\mathbf{q}}+n_{\mathbf{p}_{1}}+1\right) \frac{1}{\Omega_{4}^{2}-\Omega_{7}^{2}}\left(\Omega_{7} \operatorname{coth}\left(\frac{\beta \Omega_{4}}{2}\right)-\Omega_{4} \operatorname{coth}\left(\frac{\beta \Omega_{7}}{2}\right)\right) \\
& \times\left[\cos \Omega_{7}\left(t_{0}-t^{\prime \prime}\right) \cos \Omega_{4}\left(t_{0}-t^{\prime}\right)+\cos \Omega_{7}\left(t_{0}-t^{\prime}\right) \cos \Omega_{4}\left(t_{0}-t^{\prime \prime}\right)\right] \\
& -\frac{1}{2}\left(n_{\mathbf{k}+\mathbf{q}}-n_{\mathbf{k}}\right)\left(n_{-\mathbf{p}_{1}+\mathbf{q}}+n_{\mathbf{p}_{1}}+1\right) \frac{1}{\Omega_{4}^{2}-\Omega_{7}^{2}}\left(-\Omega_{7} \operatorname{coth}\left(\frac{\beta \Omega_{7}}{2}\right)+\Omega_{4} \operatorname{coth}\left(\frac{\beta \Omega_{4}}{2}\right)\right) \\
& \times\left[\sin \Omega_{7}\left(t_{0}-t^{\prime \prime}\right) \sin \Omega_{4}\left(t_{0}-t^{\prime}\right)+\sin \Omega_{7}\left(t_{0}-t^{\prime}\right) \sin \Omega_{4}\left(t_{0}-t^{\prime \prime}\right)\right] \\
& -\frac{1}{2}\left(n_{\mathbf{k}+\mathbf{q}}+n_{\mathbf{k}}+1\right)\left(n_{-\mathbf{p}_{1}+\mathbf{q}}-n_{\mathbf{p}_{1}}\right) \frac{1}{\Omega_{3}^{2}-\Omega_{8}^{2}}\left(\Omega_{8} \operatorname{coth}\left(\frac{\beta \Omega_{3}}{2}\right)-\Omega_{3} \operatorname{coth}\left(\frac{\beta \Omega_{8}}{2}\right)\right) \\
& \times\left[\cos \Omega_{8}\left(t_{0}-t^{\prime \prime}\right) \cos \Omega_{3}\left(t_{0}-t^{\prime}\right)+\cos \Omega_{8}\left(t_{0}-t^{\prime}\right) \cos \Omega_{3}\left(t_{0}-t^{\prime \prime}\right)\right] \\
& -\frac{1}{2}\left(n_{\mathbf{k}+\mathbf{q}}+n_{\mathbf{k}}+1\right)\left(n_{-\mathbf{p}_{1}+\mathbf{q}}-n_{\mathbf{p}_{1}}\right) \frac{1}{\Omega_{3}^{2}-\Omega_{8}^{2}}\left(\Omega_{3} \operatorname{coth}\left(\frac{\beta \Omega_{3}}{2}\right)-\Omega_{8} \operatorname{coth}\left(\frac{\beta \Omega_{8}}{2}\right)\right) \\
& \times\left[\sin \Omega_{8}\left(t_{0}-t^{\prime \prime}\right) \sin \Omega_{3}\left(t_{0}-t^{\prime}\right)+\sin \Omega_{8}\left(t_{0}-t^{\prime}\right) \sin \Omega_{3}\left(t_{0}-t^{\prime \prime}\right)\right] \\
& +\frac{1}{2}\left(n_{\mathbf{k}+\mathbf{q}}-n_{\mathbf{k}}\right)\left(n_{-\mathbf{p}_{1}+\mathbf{q}}-n_{\mathbf{p}_{1}}\right) \frac{1}{\Omega_{4}^{2}-\Omega_{8}^{2}}\left(\Omega_{8} \operatorname{coth}\left(\frac{\beta \Omega_{4}}{2}\right)-\Omega_{4} \operatorname{coth}\left(\frac{\beta \Omega_{8}}{2}\right)\right) \\
& \times\left[\cos \Omega_{8}\left(t_{0}-t^{\prime \prime}\right) \cos \Omega_{4}\left(t_{0}-t^{\prime}\right)+\cos \Omega_{8}\left(t_{0}-t^{\prime}\right) \cos \Omega_{4}\left(t_{0}-t^{\prime \prime}\right)\right] \\
& +\frac{1}{2}\left(n_{\mathbf{k}+\mathbf{q}}-n_{\mathbf{k}}\right)\left(n_{-\mathbf{p}_{1}+\mathbf{q}}-n_{\mathbf{p}_{1}}\right) \frac{1}{\Omega_{4}^{2}-\Omega_{8}^{2}}\left(\Omega_{4} \operatorname{coth}\left(\frac{\beta \Omega_{4}}{2}\right)-\Omega_{8} \operatorname{coth}\left(\frac{\beta \Omega_{8}}{2}\right)\right) \\
& \times\left[\sin \Omega_{8}\left(t_{0}-t^{\prime \prime}\right) \sin \Omega_{4}\left(t_{0}-t^{\prime}\right)+\sin \Omega_{8}\left(t_{0}-t^{\prime}\right) \sin \Omega_{4}\left(t_{0}-t^{\prime \prime}\right)\right]
\end{aligned}
$$

When we optimize with respect to initial state, the second term of (3.143) $\operatorname{tr}\left[l^{\Phi \Phi}\left(t^{\prime}, t_{0}\right) X\left(t_{0}, t^{\prime \prime}\right)\right]$ cancels exactly the term $P^{(1)}$. One checks also that at $t^{\prime}=t^{\prime \prime}=t_{0}$, this second term is vanishing.

In order to obtain the expression of $\Pi\left(\mathbf{q}^{2}, t^{\prime}, t^{\prime \prime}\right)$, we have used a symmetry in the exchange of $\mathbf{k}$ and $-\mathbf{p}_{1}$ of the function to integrate in $\Pi\left(\mathbf{q}^{2}, t^{\prime}, t^{\prime \prime}\right)$. It is important to note that the function $\int \frac{d^{3} p_{1}}{(2 \pi)^{3}} \Sigma_{11}^{\Phi \Phi}\left(\mathbf{k},-\mathbf{q}-\mathbf{k}, \mathbf{p}_{1}, \mathbf{q}-\mathbf{p}_{1}, t^{\prime}, t^{\prime \prime}\right)$ remains dependent on $t_{0}$.

For $t^{\prime}>t^{\prime \prime}$, expression (3.143) becomes :

$$
\begin{aligned}
& \Pi\left(\mathbf{q}^{2}, t^{\prime}, t^{\prime \prime}\right)=\frac{i}{2} \int \frac{d^{3} k}{(2 \pi)^{3}} \frac{d^{3} p_{1}}{(2 \pi)^{3}} \frac{d^{3} p}{(2 \pi)^{3}} \times \\
& \operatorname{tr}\left[l^{\Phi \Phi}\left(\mathbf{p}+\mathbf{q},-\mathbf{p}, \mathbf{k},-\mathbf{q}-\mathbf{k}, t^{\prime \prime}, t_{0}\right)\left(\bar{\Xi}_{0}(\mathbf{p})-\tau\right) l^{\Phi \Phi}\left(\mathbf{p},-\mathbf{p}-\mathbf{q}, \mathbf{p}_{1}, \mathbf{q}-\mathbf{p}_{1}, t^{\prime}, t_{0}\right)\left(\bar{\Xi}_{0}(\mathbf{p}+\mathbf{q})+\tau\right)\right] \\
& -\operatorname{tr}\left[l^{\Phi \Phi}\left(\mathbf{p}+\mathbf{q},-\mathbf{p}, \mathbf{k},-\mathbf{q}-\mathbf{k}, t^{\prime \prime}, t_{0}\right) X\left(t_{0}, t^{\prime}\right)\right]
\end{aligned}
$$


and one shows that one obtains $\Pi^{(1)}\left(\mathbf{q}^{2}, \Delta t\right)$ from (3.144) by replacing $\Delta t$ by $-\Delta t$.

Similarly to the case of the Green function with two field operators $\Phi(\mathbf{x}) \Phi(\mathbf{y})$, we can check the fluctuation-dissipation theorem for the Green function with four field operators $\Phi^{2}(\mathbf{x}) \Phi^{2}(\mathbf{y})$. The expression of $\Pi\left(\mathbf{q}^{2}, \tau\right)$ for $\Delta t>0$ is indeed equivalent to expression (3.144) of $\Pi\left(\mathbf{q}^{2},-\tau-i \beta\right)$ for $\Delta t<0$.

Let us study the retarded Green function with two field operators $\chi_{\Phi \Phi, \Phi \Phi}\left(t^{\prime}, t^{\prime \prime}\right)$ defined by (2.120) when we optimize with respect to the initial state. For $t^{\prime}>t^{\prime \prime}$ and with the time-ordered product :

$$
\begin{aligned}
\Sigma_{11}^{\Phi \Phi}\left(\mathbf{k},-\mathbf{q}-\mathbf{k}, \mathbf{p}_{1}, \mathbf{q}-\mathbf{p}_{1}, t^{\prime}, t^{\prime \prime}\right)= & -\int \frac{d^{3} p}{(2 \pi)^{3}} \operatorname{tr}\left[l^{\Phi \Phi}\left(\mathbf{p}-\mathbf{q},-\mathbf{p}, \mathbf{k},-\mathbf{q}-\mathbf{k}, t^{\prime \prime}, t_{0}\right)\right. \\
& \left.\Sigma^{\Phi \Phi}\left(\mathbf{p},-\mathbf{p}-\mathbf{q}, \mathbf{p}_{1}, \mathbf{q}-\mathbf{p}_{1}, t_{0}, t^{\prime}\right)\right]
\end{aligned}
$$

For $t^{\prime}>t^{\prime \prime}$ and the anti-T product :

$$
\begin{aligned}
\Sigma_{11}^{\Phi \Phi}\left(\mathbf{k},-\mathbf{q}-\mathbf{k}, \mathbf{p}_{1}, \mathbf{q}-\mathbf{p}_{1}, t^{\prime}, t^{\prime \prime}\right)= & -\int \frac{d^{3} p}{(2 \pi)^{3}} \operatorname{tr}\left[l^{\Phi \Phi}\left(\mathbf{p}-\mathbf{q},-\mathbf{p}, \mathbf{k},-\mathbf{q}-\mathbf{k}, t^{\prime}, t_{0}\right)\right. \\
& \left.\Sigma^{\Phi \Phi}\left(\mathbf{p},-\mathbf{p}-\mathbf{q}, \mathbf{p}_{1}, \mathbf{q}-\mathbf{p}_{1}, t_{0}, t^{\prime \prime}\right)\right]
\end{aligned}
$$

We use formula (3.134) obtained at the first order in perturbation. The retarded Green function $\chi_{\Phi \Phi, \Phi \Phi}\left(t^{\prime}, t^{\prime \prime}\right)$ is given by expression (2.121) obtained by optimizing only the dynamics and a term equal to :

$$
\begin{aligned}
& -\int \frac{d^{3} p}{(2 \pi)^{3}} \operatorname{tr}\left[l^{\Phi \Phi}\left(\mathbf{p}-\mathbf{q},-\mathbf{p}, \mathbf{k},-\mathbf{q}-\mathbf{k}, t^{\prime \prime}, t_{0}\right) X\left(t_{0}, t^{\prime}\right)\right] \\
& +\int \frac{d^{3} p}{(2 \pi)^{3}} \operatorname{tr}\left[l^{\Phi \Phi}\left(\mathbf{p}-\mathbf{q},-\mathbf{p}, \mathbf{k},-\mathbf{q}-\mathbf{k}, t^{\prime \prime}, t_{0}\right) X\left(t_{0}, t^{\prime}\right)\right]
\end{aligned}
$$

where $\operatorname{tr}\left[l^{\Phi \Phi}\left(t^{\prime}, t_{0}\right) X\left(t_{0}, t^{\prime \prime}\right)\right]$ is equal to the term $P^{(1)}$ (eq. (3.147)). We see from the expression of $P^{(1)}$ by interchanging $t^{\prime}$ and $t^{\prime \prime}$ that the difference $\operatorname{tr}\left[l^{\Phi \Phi}\left(t^{\prime \prime}, t_{0}\right) X\left(t_{0}, t^{\prime}\right)\right]-$ $\operatorname{tr}\left[l^{\Phi \Phi}\left(t^{\prime}, t_{0}\right) X\left(t_{0}, t^{\prime \prime}\right)\right]$ is vanishing.

To conclude, for the the linear response formula which involves the retarded Green functions, we obtain the same result with or without the optimization with respect to initial state. It is sufficient to solve the backward dynamical equations (3.114 3.117) in real time for $l^{\Phi \Phi}$. 
The first order contribution to the polarization function $\Pi_{R}$ writes :

$$
\begin{aligned}
\Pi_{R}^{(1)} & \left(\mathbf{q}^{2}, t^{\prime}, t^{\prime \prime}\right)=-\theta\left(t^{\prime}-t^{\prime \prime}\right) \frac{b}{4} \int \frac{d^{3} p}{(2 \pi)^{3}} \frac{1}{\omega_{\mathbf{p}} \omega_{\mathbf{p}+\mathbf{q}}} \times \\
& {\left[\left(n_{\mathbf{p}+\mathbf{q}}+n_{\mathbf{p}}+1\right) \sin \Omega_{1} \Delta t \int \frac{d^{3} k}{(2 \pi)^{3}} \frac{1}{\omega_{\mathbf{k}} \omega_{\mathbf{q}-\mathbf{k}}}\left[\left(n_{\mathbf{q}-\mathbf{k}}+n_{\mathbf{k}}+1\right) \frac{\Omega_{3}}{\Omega_{1}^{2}-\Omega_{3}^{2}}-\left(n_{\mathbf{q}-\mathbf{k}}-n_{\mathbf{k}}\right) \frac{\Omega_{4}}{\Omega_{1}^{2}-\Omega_{4}^{2}}\right]\right.} \\
& \left.-\left(n_{\mathbf{p}+\mathbf{q}}-n_{\mathbf{p}}\right) \sin \Omega_{2} \Delta t \int \frac{d^{3} k}{(2 \pi)^{3}} \frac{1}{\omega_{\mathbf{k}} \omega_{\mathbf{q}-\mathbf{k}}}\left[\left(n_{\mathbf{q}-\mathbf{k}}+n_{\mathbf{k}}+1\right) \frac{\Omega_{3}}{\Omega_{2}^{2}-\Omega_{3}^{2}}-\left(n_{\mathbf{q}-\mathbf{k}}-n_{\mathbf{k}}\right) \frac{\Omega_{4}}{\Omega_{2}^{2}-\Omega_{4}^{2}}\right]\right]
\end{aligned}
$$

with

$$
\begin{array}{ll}
\Omega_{1}=\omega_{\mathbf{p}+\mathbf{q}}+\omega_{\mathbf{p}} & \Omega_{2}=\omega_{\mathbf{p}+\mathbf{q}}-\omega_{\mathbf{p}} \\
\Omega_{3}=\omega_{\mathbf{q}-\mathbf{k}}+\omega_{\mathbf{k}} & \Omega_{4}=\omega_{\mathbf{q}-\mathbf{k}}-\omega_{\mathbf{k}}
\end{array}
$$

and $\Delta t=t^{\prime}-t^{\prime \prime}$

By taking its Fourrier transform in time and adding the zero order term and the first order term, we obtain :

$$
\begin{aligned}
& \Pi_{R}\left(\mathbf{q}^{2}, \omega\right)=\int \frac{d^{3} p}{(2 \pi)^{3}} \frac{1}{\omega_{\mathbf{p}} \omega_{\mathbf{p}+\mathbf{q}}} \times \\
& {\left[\left(n_{\mathbf{p}+\mathbf{q}}+n_{\mathbf{p}}+1\right) \frac{\Omega_{1}}{(\omega+i \eta)^{2}-\Omega_{1}^{2}}\right.} \\
& \left(1+\frac{b}{4} \int \frac{d^{3} k}{(2 \pi)^{3}} \frac{1}{\omega_{\mathbf{k}} \omega_{\mathbf{q}-\mathbf{k}}}\left[\left(n_{\mathbf{q}-\mathbf{k}}+n_{\mathbf{k}}+1\right) \frac{\Omega_{3}}{\Omega_{1}^{2}-\Omega_{3}^{2}}-\left(n_{\mathbf{q}-\mathbf{k}}-n_{\mathbf{k}}\right) \frac{\Omega_{4}}{\Omega_{1}^{2}-\Omega_{4}^{2}}\right]\right) \\
& -\left(n_{\mathbf{p}+\mathbf{q}}-n_{\mathbf{p}}\right) \frac{\Omega_{2}}{(\omega+i \eta)^{2}-\Omega_{2}^{2}} \\
& \left.\left(1+\frac{b}{4} \int \frac{d^{3} k}{(2 \pi)^{3}} \frac{1}{\omega_{\mathbf{k}} \omega_{\mathbf{q}-\mathbf{k}}}\left[\left(n_{\mathbf{q}-\mathbf{k}}+n_{\mathbf{k}}+1\right) \frac{\Omega_{3}}{\Omega_{2}^{2}-\Omega_{3}^{2}}-\left(n_{\mathbf{q}-\mathbf{k}}-n_{\mathbf{k}}\right) \frac{\Omega_{4}}{\Omega_{2}^{2}-\Omega_{4}^{2}}\right]\right)\right]
\end{aligned}
$$

Although the coupling constant appears linearly in the numerator, this formula includes nonperturbative contributions since the frequencies involves the self-consistent renormalized mass $m^{2}(\beta)$ eq. (2.66).

The divergent part of the integrals at high momentum is entirely determined by the contribution at $T=0$. This one writes :

$$
\begin{aligned}
\Pi_{R}\left(\mathbf{q}^{2}, \omega\right)= & \int \frac{d^{3} p}{(2 \pi)^{3}} \frac{1}{\omega_{\mathbf{p}} \omega_{\mathbf{p}+\mathbf{q}}} \frac{\omega_{\mathbf{p}+\mathbf{q}}+\omega_{\mathbf{p}}}{(\omega+i \eta)^{2}-\left(\omega_{\mathbf{p}+\mathbf{q}}+\omega_{\mathbf{p}}\right)^{2}} \times \\
& \left(1+\frac{b}{4} \int \frac{d^{3} k}{(2 \pi)^{3}} \frac{1}{\omega_{\mathbf{k}} \omega_{\mathbf{q}+\mathbf{k}}} \frac{\omega_{\mathbf{q}+\mathbf{k}}+\omega_{\mathbf{k}}}{\left(\omega_{\mathbf{p}+\mathbf{q}}+\omega_{\mathbf{p}}\right)^{2}-\left(\omega_{\mathbf{q}+\mathbf{k}}+\omega_{\mathbf{k}}\right)^{2}}\right)
\end{aligned}
$$

(We remark that there is no term in in the denominator of the second integral.)

At zero temperature, $\Pi_{R}\left(\mathbf{q}^{2}, \omega\right)$ depends only on the variable $s=\omega^{2}-\mathbf{q}^{2}$. At the lowest order,

$$
\Pi_{R}^{(0)}(s)=\int \frac{d^{3} p}{(2 \pi)^{3}} \frac{\omega_{\mathbf{p}+\mathbf{q}}+\omega_{\mathbf{p}}}{\omega_{\mathbf{p}} \omega_{\mathbf{p}+\mathbf{q}}} \frac{1}{(\omega+i \eta)^{2}-\left(\omega_{\mathbf{p}+\mathbf{q}}+\omega_{\mathbf{p}}\right)^{2}}
$$


which is related to the explicitly Lorentz invariant form :

$$
\Pi_{F}(s)=i \int \frac{d^{4} p}{(2 \pi)^{4}} \frac{1}{p^{2}-\bar{m}^{2}+i \epsilon} \frac{1}{(p+q)^{2}-\bar{m}^{2}+i \epsilon}
$$

where $q=(\omega, \mathbf{q})$. We have $\operatorname{Re} \Pi_{R}^{(0)}(s)=\operatorname{Re} \Pi_{F}(s)$ and $\operatorname{Im} \Pi_{R}^{(0)}(s)=\operatorname{sign}(\omega) \operatorname{Im} \Pi_{F}(s) . \bar{m}$ is the renormalized self-consistent mass at zero temperature given by eq. (2.64). By using for instance the results of Kerman and Lin [16],

$$
\Pi_{R}^{(0)}(s)=-\frac{1}{4 \pi^{2}} \log \left(\frac{2 \Lambda}{\sqrt{e} \mu}\right)+\frac{1}{4 \pi^{2}} \log \left(\frac{\bar{m}}{\mu}\right)-\frac{1}{8 \pi^{2}} f(s)-\theta\left(s-4 \bar{m}^{2}\right) \frac{i}{8 \pi} \sqrt{\frac{s-4 \bar{m}^{2}}{s}}
$$

where $\Lambda$ is a momemtum cut-off, $\mu$ is an arbitrary mass scale and the function $f(s)$ is defined by :

$$
\begin{gathered}
f(s)=2+\sqrt{\frac{s-4 \bar{m}^{2}}{s}} \log \frac{\sqrt{s}-\sqrt{s-4 \bar{m}^{2}}}{\sqrt{s+\sqrt{s-4 \bar{m}^{2}}}} \quad \text { si } \quad s>4 \bar{m}^{2} \\
f(s)=2-2 \sqrt{\frac{-4 \bar{m}^{2}}{s}} \tan ^{-1} \sqrt{\frac{s}{-4 \bar{m}^{2}}} \quad \text { si } \quad 0<s<4 \bar{m}^{2} \\
f(s)=2+\sqrt{\frac{s-4 \bar{m}^{2}}{s}} \log \frac{\sqrt{s-4 \bar{m}^{2}}-\sqrt{s}}{\sqrt{s+4 \bar{m}^{2}}+\sqrt{s}} \quad \text { si } \quad s<0
\end{gathered}
$$

Adding the zero order term and the first order term, we have at zero temperature :

$$
\Pi_{R}(s)=\Pi_{R}^{(0)}(s)\left(1-\frac{b}{16 \pi^{2}} \log \left(\frac{2 \Lambda}{\sqrt{e} \mu}\right)\right)
$$

and $\Pi_{R}^{(0)}(s)$ is given by expression (3.159). We see that at the order we have considered, the logarithmic divergence appears squared. By resumming the perturbative serie, we obtain :

$$
\Pi_{R}(s)=\frac{\Pi_{R}^{(0)}(s)}{1+\frac{b}{16 \pi^{2}} \log \left(\frac{2 \Lambda}{\sqrt{e} \mu}\right)}
$$

We can define a renormalized coupling constant $g_{R}(\mu)$ by

$$
2 g_{R}(\mu) \Pi_{R}^{(0)}(s)=b \Pi_{R}(s)
$$

i. e. :

$$
\frac{1}{2 g_{R}(\mu)}=\frac{1}{b}+\frac{1}{16 \pi^{2}} \log \left(\frac{2 \Lambda}{e \sqrt{\mu}}\right)
$$

This renormalization is identical to the one obtained by Kerman and Lin in the symmetric phase 16]. 


\section{Conclusion}

In this paper, we have shown how to derive variational approximations for two-time correlation functions in $\Phi^{4}$ theory. We have studied in particular the two-time correlation functions with two field operators $\Phi(\mathbf{x})$ and with four field operators $\Phi(\mathbf{x})$ in the symmetric phase. By using the variational principle introduced by Balian and Veneroni [6], we have shown how to take into account correlations both in the dynamics and in the initial state while keeping uncorrelated ansätze for the variational objects. The calculation sheme we have presented is valid for arbitrary time-dependent problems. As an illustration, we have calculated explicitly the two-time correlation functions with two field operators and with four field operators at equilibrium in the symmetric phase. The approximation we have obtained for the two field operators correlation function is identical in this case with the usual mean-field result. This won't be true in the asymmetric phase or for a time-dependent problem. The approximation for the two-time and four field operators correlation function contains already in the simple case we have considered corrections to the standard mean-field result. For the non-retarded correlation functions, it is important to optimize with respect to the initial state in order not to obtain a spurious dependence on the initial time. However, for the retarded correlation functions, the same result is obtained whether or not we optimize with respect to the initial state. Our variational approximations for the two-time correlation functions satisfy the fluctuation-dissipation theorem. For the four field operators correlation function, we were obliged to solve the dynamical equations perturbatively. The zero order approximation was already known in the litterature but we have shown here how to derive it variationally. We have calculated also the first order approximation. At zero temperature, it is possible to resum the perturbative serie and to define in this way a renormalized coupling constant. We stress that our approximations remain nonperturbative since they involve a self-consistent renormalized mass.

There are several important questions for future works. First, how can one obtain variational approximations which include damping. It would be very interesting to obtain a variational nonperturbative approximation for the viscosity defined in eq. (3.86). Damping effects are probably related to a non symmetrical form in $\mathbf{x}$ and $\mathbf{y}$ for the matrix $T$ defined in eq. (2.24) to characterize our gaussian variational ansätze and to a choice for the operator $\bar{H}$ different from the Hamiltonian $H$. A second interesting problem is the evolution of a scalar field in self-interaction in a time-dependent metric. The difficulty in this case is to solve the backward dynamical equations in order to obtain variational 
approximations for the two-time correlation functions.

\section{Aknowledgements}

I would like to thank Roger Balian and Marcel Vénéroni for very fruitful discussions. I thank also Olivier Martin for a critical reading of a part of the manuscript.

\section{Appendix A : Characterization of a Gaussian state}

We call a Gaussian operator an operator which is an exponential of quadratic and linear forms of the field operators $\Phi(\mathbf{x})$ and $\Pi(\mathbf{x})$. A Gaussian operator $\mathcal{D}(t)$ is completely characterized by the vector $\alpha(\mathbf{x}, t)$ and the matrix $\Xi(\mathbf{x}, \mathbf{y}, t)$ defined by the equations (2.18)-(2.25) of the section 2.3.

Instead of the quantities $G, T$ and $S$, the authors of reference [9] have introduced the quantities $G_{\xi}, \Sigma_{\xi}$ and $\xi, \xi$ being the degree of mixing (for a pur state, $\xi=1$ ):

$$
\begin{gathered}
\operatorname{Tr}(\mathcal{D}(t) \bar{\Phi}(\mathbf{x}) \bar{\Phi}(\mathbf{y}))=\left(G_{\xi}^{1 / 2}(1-\xi)^{-1} G_{\xi}^{1 / 2}\right)(\mathbf{x}, \mathbf{y}, t) \\
\operatorname{Tr}(\mathcal{D}(t) \bar{\Pi}(\mathbf{x}) \bar{\Pi}(\mathbf{y}))=\frac{1}{4}\left(G_{\xi}^{-1 / 2}(1+\xi) G_{\xi}^{-1 / 2}\right)(\mathbf{x}, \mathbf{y}, t)+4\left(\Sigma_{\xi} G_{\xi}^{1 / 2}(1-\xi)^{-1} G_{\xi}^{1 / 2} \Sigma_{\xi}\right)(\mathbf{x}, \mathbf{y}, t) \\
\operatorname{Tr}(\mathcal{D}(t) \bar{\Phi}(\mathbf{x}) \bar{\Pi}(\mathbf{y}))=\frac{i}{2} \delta^{3}(\mathbf{x}-\mathbf{y})+2\left(G_{\xi}^{1 / 2}(1-\xi)^{-1} G_{\xi}^{1 / 2} \Sigma_{\xi}\right)(\mathbf{x}, \mathbf{y}, t)
\end{gathered}
$$

It is usefull to relate our notations to those of the authors of [9]. For a pure state $(\xi=0)$,

$$
\begin{gathered}
T(\mathbf{x}, \mathbf{y}, t)=2(G \Sigma+\Sigma G)(\mathbf{x}, \mathbf{y}, t) \\
S(\mathbf{x}, \mathbf{y}, t)=\frac{1}{4} G^{-1}(\mathbf{x}, \mathbf{y}, t)+4(\Sigma G \Sigma)(\mathbf{x}, \mathbf{y}, t) .
\end{gathered}
$$

For an uniform configuration, we have the following relations :

$$
\begin{gathered}
G(\mathbf{p})=G_{\xi}(\mathbf{p})(1-\xi(\mathbf{p}))^{-1} \\
S(\mathbf{p})=\frac{1}{4} G_{\xi}^{-1}(\mathbf{p})(1+\xi(\mathbf{p}))+4 \Sigma_{\xi}^{2}(\mathbf{p}) G_{\xi}(\mathbf{p}) \frac{1}{1-\xi(\mathbf{p})} \\
T(\mathbf{p})=4 G_{\xi}(\mathbf{p}) \frac{1}{1-\xi(\mathbf{p})} \Sigma_{\xi}(\mathbf{p})
\end{gathered}
$$

and the Heisenberg invariant is related to the degree of mixing according to :

$$
I(\mathbf{p})=\frac{1+\xi(\mathbf{p})}{1-\xi(\mathbf{p})}
$$




\section{Appendix B : Expression of the von Neuman entropy for bosons in the} Hartree-Bogoliubov approximation

The von-Neuman entropy is given as a function of the density operator $\mathcal{D}$ by :

$$
S=-\frac{\operatorname{Tr} \mathcal{D} \log \mathcal{D}}{\operatorname{Tr} \mathcal{D}}+\log \operatorname{Tr} \mathcal{D}
$$

In the $\mathrm{HB}$ approximation, $\mathcal{D}$ is the exponential of linear and quadratic forms of the field operator $\Phi$ and $\Pi$. The entropy density then writes in terms of the matrix $\Xi$ :

$$
\begin{aligned}
\mathcal{S}=\int \frac{d^{3} p}{(2 \pi)^{3}} \quad & \frac{1}{4} \operatorname{tr}\left\{\left(\frac{1}{2}(1-\tau) \Xi(1-\tau)-1\right) \log \left(\frac{\Xi+\tau}{\Xi-\tau}\right)\right\} \\
& +\frac{1}{2} \log \operatorname{det}\left\{\frac{1}{4}\left(\begin{array}{cc}
1 & 1 \\
1 & -1
\end{array}\right) \Xi(1-\tau)-\frac{1}{2}\left(\begin{array}{ll}
0 & 1 \\
1 & 0
\end{array}\right)\right\} .
\end{aligned}
$$

where $\tau$ is the $2 \times 2$ matrix

$$
\tau=\left(\begin{array}{cc}
0 & 1 \\
-1 & 0
\end{array}\right)
$$

It can also be written in terms of the Heisenberg invariant I (2.26) :

$$
\mathcal{S}=\int \frac{d^{3} p}{(2 \pi)^{3}}\left[\frac{1}{2} \sqrt{I} \log \left(\frac{\sqrt{I}+1}{\sqrt{I}-1}\right)+\frac{1}{2} \log (I-1)\right] .
$$

For the static HB solution characterized by $\alpha^{0}, \Xi^{0}$, we have : $I=-\Xi_{11}^{0} \Xi_{22}^{0}$. It is easy to convince oneself that expression (B.4) is in this case identical to :

$$
S=\int \frac{d^{3} p}{(2 \pi)^{3}}\left[\left(n_{\mathbf{p}}+1\right) \log \left(n_{\mathbf{p}}+1\right)-n_{\mathbf{p}} \log n_{\mathbf{p}}\right]
$$

where the occupation number $n_{\mathbf{p}}$ is related to the degree of mixing $\xi(p)$ according to :

$$
2 n_{\mathbf{p}}+1=\sqrt{\frac{1+\xi(\mathbf{p})}{1-\xi(\mathbf{p})}}=\sqrt{I(\mathbf{p})} .
$$

By using the parametrization $\xi(\mathbf{p})=\frac{1}{\cosh \beta \omega_{\mathbf{p}}}$, we have also : $2 n_{\mathbf{p}}+1=\operatorname{coth}\left(\frac{\beta \omega_{\mathbf{p}}}{2}\right)$. In the $\Phi^{4}$ theory, $\omega_{\mathbf{p}}=\sqrt{-g_{0}(\mathbf{p})}$ and

$$
g_{0}(\mathbf{p})=-\left(\mathbf{p}^{2}+m_{0}^{2}+\frac{b}{2} \varphi_{0}^{2}+\frac{b}{2} G_{0}(\mathbf{x}, \mathbf{x})\right) .
$$

The static solution satisfies:

$$
\Xi_{11}^{0}(\mathbf{p}) g_{0}(\mathbf{p})=\Xi_{22}^{0}(\mathbf{p})
$$


and

$$
\Xi_{11}^{0}(\mathbf{p}) \Xi_{22}^{0}(\mathbf{p})=-\frac{1+\xi(\mathbf{p})}{1-\xi(\mathbf{p})}
$$

In terms of the frequency $\omega_{\mathbf{p}}$ :

$$
\begin{gathered}
\Xi_{11}^{0}(\mathbf{p})=\frac{1}{\omega_{\mathbf{p}}} \operatorname{coth}\left(\frac{\beta}{2} \omega_{\mathbf{p}}\right) \\
\Xi_{22}^{0}(\mathbf{p})=-\omega_{\mathbf{p}} \operatorname{coth}\left(\frac{\beta}{2} \omega_{\mathbf{p}}\right) \\
I(\mathbf{p})=\operatorname{coth}^{2}\left(\frac{\beta}{2} \omega_{\mathbf{p}}\right)
\end{gathered}
$$

The matrix of the second derivatives of the entropy evaluated for the static solution $\Xi_{0}$ is given by the following elements :

$$
\begin{gathered}
\left.\frac{\partial^{2} \mathcal{S}}{\partial \Xi_{11}(\mathbf{p}) \partial \Xi_{11}(\mathbf{p})}\right|_{\Xi_{0}}=-\frac{\omega_{\mathbf{p}}^{2}}{4}\left[\frac{x}{\operatorname{coth} x}+\frac{1}{\operatorname{coth}^{2} x-1}\right] \\
\left.\frac{\partial^{2} \mathcal{S}}{\partial \Xi_{11}(\mathbf{p}) \partial \Xi_{22}(\mathbf{p})}\right|_{\Xi_{0}}=-\frac{1}{4}\left[\frac{x}{\operatorname{coth} x}-\frac{1}{\operatorname{coth}^{2} x-1}\right] \\
\left.\frac{\partial^{2} \mathcal{S}}{\partial \Xi_{22}(\mathbf{p}) \partial \Xi_{22}(\mathbf{p})}\right|_{\Xi_{0}}=-\frac{1}{4 \omega_{\mathbf{p}}^{2}}\left[\frac{x}{\operatorname{coth} x}+\frac{1}{\operatorname{coth}^{2} x-1}\right] \\
\left.\frac{\partial^{2} \mathcal{S}}{\partial \Xi_{12}(\mathbf{p}) \partial \Xi_{12}(\mathbf{p})}\right|_{\Xi_{0}}=\frac{x}{\operatorname{coth} x}
\end{gathered}
$$

where we have introduced the notation $x=\beta \omega_{\mathbf{p}} / 2$.

\section{Appendix C : Parametrization of the product of two Gaussians}

In this appendix, we give the expressions of the expectation values $\alpha_{b}, \alpha_{c}, \Xi_{b}$ and $\Xi_{c}$ in terms of $\alpha_{d}, \alpha_{a}, \Xi_{d}$ and $\Xi_{a}$.

$$
\begin{aligned}
& \alpha_{b}=\left(\Xi_{a}-\tau\right) \frac{1}{\Xi_{a}+\Xi_{d}} \alpha_{d}+\left(\Xi_{d}+\tau\right) \frac{1}{\Xi_{a}+\Xi_{d}} \alpha_{a}, \\
& \alpha_{c}=\left(\Xi_{d}-\tau\right) \frac{1}{\Xi_{a}+\Xi_{d}} \alpha_{a}+\left(\Xi_{a}+\tau\right) \frac{1}{\Xi_{a}+\Xi_{d}} \alpha_{d},
\end{aligned}
$$

where $\tau$ is $2 \times 2$ matrix :

$$
\tau=\left(\begin{array}{cc}
0 & 1 \\
-1 & 0
\end{array}\right)
$$

For the matrices $\Xi$, we have the following relations :

$$
\Xi_{b}-\tau=\left(\Xi_{a}-\tau\right) \frac{1}{\Xi_{a}+\Xi_{d}}\left(\Xi_{d}-\tau\right),
$$




$$
\begin{aligned}
& \Xi_{c}-\tau=\left(\Xi_{d}-\tau\right) \frac{1}{\Xi_{a}+\Xi_{d}}\left(\Xi_{a}-\tau\right) . \\
& \Xi_{b}+\tau=\left(\Xi_{d}+\tau\right) \frac{1}{\Xi_{a}+\Xi_{d}}\left(\Xi_{a}+\tau\right), \\
& \Xi_{c}+\tau=\left(\Xi_{a}+\tau\right) \frac{1}{\Xi_{a}+\Xi_{d}}\left(\Xi_{d}+\tau\right) .
\end{aligned}
$$

\section{Appendix D}

In this appendix, we give the evolution equations for $n_{d}, \alpha_{d}, \Xi_{d} n_{a}, \alpha_{a}, \Xi_{a}$.

$$
\begin{gathered}
i \frac{\dot{n}_{d}}{n_{d}}=F_{c}^{(d)}-F_{b}^{(d)}-F_{K c}^{(d)} \\
2 i \dot{\alpha}_{d}=-\left[\left(\Xi_{d}+\tau\right)\left(\mathcal{H}_{c}-\mathcal{I}_{K}^{c}\right)\left(\Xi_{d}-\tau\right)-\left(\Xi_{d}-\tau\right) \mathcal{H}_{b}\left(\Xi_{d}+\tau\right)\right] \tau \alpha_{b-c} \\
+\Xi_{d}\left(w_{c}-w_{b}\right)+\tau\left(w_{c}+w_{b}\right)-\left(\Xi_{d}+\tau\right) w_{K}^{c} \\
i \dot{\Xi}_{d}=-\left[\left(\Xi_{d}+\tau\right)\left(\mathcal{H}_{c}-\mathcal{I}_{K}^{c}\right)\left(\Xi_{d}-\tau\right)-\left(\Xi_{d}-\tau\right) \mathcal{H}_{b}\left(\Xi_{d}+\tau\right)\right] \\
2 i \dot{\alpha}_{a}=\left[\left(\Xi_{a}-\tau\right)\left(\mathcal{H}_{c}-\mathcal{I}_{K}^{c}\right)\left(\Xi_{a}+\tau\right)-\left(\Xi_{a}+\tau\right) \mathcal{H}_{b}\left(\Xi_{a}-\tau\right)\right] \tau \alpha_{c-b}(a)-F_{c}^{(a)}+F_{K c}^{(a)} \\
+\Xi_{a}\left(w_{b}-w_{c}\right)+\tau\left(w_{c}+w_{b}\right)-\left(\Xi_{a}-\tau\right) w_{K}^{c} \\
i \dot{\Xi}_{a}=\left[\left(\Xi_{a}-\tau\right)\left(\mathcal{H}_{c}-\mathcal{I}_{K}^{c}\right)\left(\Xi_{a}+\tau\right)-\left(\Xi_{a}+\tau\right) \mathcal{H}_{b}\left(\Xi_{a}-\tau\right)\right]
\end{gathered}
$$

The expressions for the vector $w$ and the matrices $\mathcal{H}$ and $\mathcal{I}$ are the following :

$$
\begin{gathered}
\tilde{w}_{b}(\mathbf{x}, t)_{1}=\frac{\delta<H>_{b}}{\delta \varphi_{b}(\mathbf{x}, t)}=-f_{b}(\mathbf{x}, t) \quad, \quad \tilde{w}_{b}(\mathbf{x}, t)_{2}=i \frac{\delta<H>_{b}}{\delta \pi_{b}(\mathbf{x}, t)}=i \pi_{b}(\mathbf{x}, t) \\
\mathcal{H}_{b}(\mathbf{x}, \mathbf{y}, t)_{i j}=-2 \frac{\delta<H>_{b}}{\delta \Xi_{b}(\mathbf{x}, \mathbf{y}, t)_{j i}} \\
\mathcal{H}_{b}(\mathbf{x}, \mathbf{y}, t)_{11}=-\frac{\delta<H>_{b}}{\delta G_{b}(\mathbf{y}, \mathbf{x}, t)}=\frac{1}{2} g_{b}(\mathbf{x}, \mathbf{y}, t) \\
\mathcal{H}_{b}(\mathbf{x}, \mathbf{y}, t)_{22}=+\frac{\delta<H>_{b}}{\delta S_{b}(\mathbf{y}, \mathbf{x}, t)}=\frac{1}{2} \delta(\mathbf{x}-\mathbf{y}) \\
\mathcal{H}_{b}(\mathbf{x}, \mathbf{y}, t)_{12}=2 i \frac{\delta<H>b}{\delta T_{b}(\mathbf{y}, \mathbf{x}, t)}=0
\end{gathered}
$$


The quantities $F$ which appear in equations (D.1) and (D.3) are defined by :

$$
\begin{aligned}
F_{b}^{(d)}= & \int d^{3} x\left(\mathcal{E}_{b}(\mathbf{x}, t)-\tilde{w}_{b}(\mathbf{x}, t) \alpha_{b-d}(\mathbf{x}, t)\right) \\
& +\frac{1}{2} \int d^{3} x d^{3} y \operatorname{tr}\left[\mathcal{H}_{b}(\mathbf{x}, \mathbf{y}, t)\left(\Xi_{b}-\Xi_{d}+2 \alpha_{b-d} \tilde{\alpha}_{b-d}\right)(\mathbf{y}, \mathbf{x}, t)\right] \\
F_{K c}^{(d)}= & \int d^{3} x\left(\mathcal{K}_{c}(\mathbf{x}, t)-\tilde{w}_{K c}(\mathbf{x}, t) \alpha_{c-d}(\mathbf{x}, t)\right) \\
& +\frac{1}{2} \int d^{3} x d^{3} y \operatorname{tr}\left[\mathcal{I}_{K c}(\mathbf{x}, \mathbf{y}, t)\left(\Xi_{c}-\Xi_{d}+2 \alpha_{c-d} \tilde{\alpha}_{c-d}\right)(\mathbf{y}, \mathbf{x}, t)\right]
\end{aligned}
$$

For the $\Phi^{4}$ theory, we have :

$$
\begin{array}{r}
f_{b}(\mathbf{x}, t)=-\left(-\Delta+m_{0}^{2}+\frac{b}{6} \varphi_{b}^{2}(\mathbf{x}, t)+\frac{b}{2} G_{b}(\mathbf{x}, \mathbf{x}, t)\right) \varphi_{b}(\mathbf{x}, t) \\
g_{b}(\mathbf{x}, \mathbf{y}, t)=-\left(-\Delta+m_{0}^{2}+\frac{b}{2} \varphi_{b}^{2}(\mathbf{x}, t)+\frac{b}{2} G_{b}(\mathbf{x}, \mathbf{x}, t)\right) \delta(\mathbf{x}-\mathbf{y}) .
\end{array}
$$

From the variations of the source term $K_{c}$, we obtain :

$$
\begin{gathered}
\tilde{w}_{K}^{c}(\mathbf{x}, t)_{1}=\frac{\delta K_{c}}{\delta \varphi_{c}(\mathbf{x}, t)}, \tilde{w}_{K}^{c}(\mathbf{x}, t)_{2}=i \frac{\delta K_{c}}{\delta \pi_{c}(\mathbf{x}, t)}, \\
\mathcal{I}_{K}^{c}(\mathbf{x}, \mathbf{y}, t)_{i j}=-2 \frac{\delta K_{c}}{\delta \Xi_{c}(\mathbf{y}, \mathbf{x}, t)_{j i}} . \\
w_{K}^{c}(\mathbf{x}, t)_{1}=J^{\Phi}(\mathbf{x}, t)+2 \int d^{3} x_{2}\left(J^{\Phi \Phi}\left(\mathbf{x}, \mathbf{x}_{2}, t\right) \varphi_{c}\left(\mathbf{x}_{2}, t\right)+J^{\Phi \Pi}\left(\mathbf{x}, \mathbf{x}_{2}, t\right) \pi_{c}\left(\mathbf{x}_{2}, t\right)\right) \\
w_{K}^{c}(\mathbf{x}, t)_{2}=i J^{\Pi}(\mathbf{x}, t)+2 i \int d^{3} x_{2}\left(J^{\Phi \Pi}\left(\mathbf{x}, \mathbf{x}_{2}, t\right) \varphi_{c}\left(\mathbf{x}_{2}, t\right)+J^{\Pi \Pi}\left(\mathbf{x}, \mathbf{x}_{2}, t\right) \pi_{c}\left(\mathbf{x}_{2}, t\right)\right) \\
\mathcal{I}_{K}^{c}(\mathbf{x}, \mathbf{y}, t)_{11}=-J^{\Phi \Phi}(\mathbf{x}, \mathbf{y}, t), \\
\mathcal{I}_{K}^{c}(\mathbf{x}, \mathbf{y}, t)_{12}=-2 i J^{\Phi \Pi}(\mathbf{x}, \mathbf{y}, t) \\
\mathcal{I}_{K}^{c}(\mathbf{x}, \mathbf{y}, t)_{22}=J^{\Pi \Pi}(\mathbf{x}, \mathbf{y}, t)
\end{gathered}
$$

The expressions for the matrices $t, T, r$ and $R$ which appear in the dynamical equations for the two-time correlation functions are the following :

$$
\begin{gathered}
t_{11}(\mathbf{x}, \mathbf{y}, t)=-g^{(0)}(\mathbf{x}, \mathbf{y}, t), t_{22}(\mathbf{x}, \mathbf{y}, t)=-\delta(\mathbf{x}-\mathbf{y}) \\
T_{1,11}(\mathbf{x}, \mathbf{y}, \mathbf{z}, t)=-\frac{b}{2} \varphi^{(0)}(\mathbf{x}, t) \delta(\mathbf{x}-\mathbf{y}) \delta^{3}(\mathbf{x}-\mathbf{z}), T_{2, j k}=0 \\
r_{11,1}(\mathbf{x}, \mathbf{y}, \mathbf{z}, t)=T_{1,11}(\mathbf{x}, \mathbf{y}, \mathbf{z}, t) \\
R_{11,11}(\mathbf{x}, \mathbf{y}, \mathbf{z}, \mathbf{u}, t)=\frac{b}{4} \delta^{3}(\mathbf{x}-\mathbf{y}) \delta^{3}(\mathbf{x}-\mathbf{z}) \delta(\mathbf{x}-\mathbf{u})
\end{gathered}
$$

The other elements are equal to zero. 
We use the following definitions for the Fourrier transform :

$$
\begin{gathered}
W\left(\mathbf{x}_{1}, \ldots, \mathbf{x}_{n}\right)=\int \frac{d^{3} p_{1}}{(2 \pi)^{3}} \ldots \frac{d^{3} p_{n}}{(2 \pi)^{3}} \exp \left(i \sum_{j=1}^{n} \mathbf{p}_{j} \cdot \mathbf{x}_{j}\right) \tilde{W}\left(\mathbf{p}_{1}, \ldots, \mathbf{p}_{n}\right) \\
\tilde{W}\left(\mathbf{p}_{1}, \ldots, \mathbf{p}_{n}\right)=\int d^{3} x_{1} \ldots d^{3} x_{n} \exp \left(-i \sum_{j=1}^{n} \mathbf{p}_{j} \cdot \mathbf{x}_{j}\right) W\left(\mathbf{x}_{1}, \ldots, \mathbf{x}_{n}\right) \\
\tilde{W}\left(\mathbf{p}_{1}, \mathbf{p}_{2}, \ldots, \mathbf{p}_{n}\right)=(2 \pi)^{3} \delta^{3}\left(\mathbf{p}_{1}+\mathbf{p}_{2}+\ldots+\mathbf{p}_{n}\right) W\left(\mathbf{p}_{1}, \mathbf{p}_{2}, \ldots, \mathbf{p}_{n}\right)
\end{gathered}
$$

So for the two-point function $\beta^{\Phi}$ :

$$
\beta_{1}^{\Phi}\left(\mathbf{x}, \mathbf{x}_{1}, t, t^{\prime \prime}\right)=\int \frac{d^{3} p}{(2 \pi)^{3}} e^{i \mathbf{p} \cdot\left(\mathbf{x}_{1}-\mathbf{x}\right)} \beta_{1}^{\Phi}\left(\mathbf{p}, t, t^{\prime \prime}\right)
$$

\section{Appendix E}

We give in this appendix the expressions of the integrals $I_{n}, J_{n}, K_{n}, L_{n}, M_{n}, N_{n}$, $n=1,2,3,4$ in termes of the constants $a, b, c, d$ introduced in (3.129) and of $l_{i j}^{\Phi \Phi}$. We introduce the frequencies

$$
\begin{gathered}
\Omega_{3}=\omega_{\mathbf{k}+\mathbf{q}}+\omega_{\mathbf{k}} \quad, \Omega_{4}=\omega_{\mathbf{k}+\mathbf{q}}-\omega_{\mathbf{k}} \\
\Omega_{5}=\omega_{\mathbf{l}+\mathbf{q}}+\omega_{\mathbf{l}} \quad, \quad \Omega_{6}=\omega_{\mathbf{l}+\mathbf{q}}-\omega_{\mathbf{l}}
\end{gathered}
$$

We define :

$$
\begin{aligned}
& I_{1}=\int \frac{d^{3} l}{(2 \pi)^{3}} \frac{a}{\Omega_{3}^{2}-\Omega_{5}^{2}} \quad, I_{2}=\int \frac{d^{3} l}{(2 \pi)^{3}} \frac{c}{\Omega_{3}^{2}-\Omega_{6}^{2}} \\
& I_{3}=\int \frac{d^{3} l}{(2 \pi)^{3}} \frac{a}{\Omega_{4}^{2}-\Omega_{5}^{2}} \quad, I_{4}=\int \frac{d^{3} l}{(2 \pi)^{3}} \frac{c}{\Omega_{4}^{2}-\Omega_{6}^{2}} \\
& J_{1}=\int \frac{d^{3} l}{(2 \pi)^{3}} \frac{b \Omega_{5}}{\Omega_{3}^{2}-\Omega_{5}^{2}} \quad, \quad J_{2}=\int \frac{d^{3} l}{(2 \pi)^{3}} \frac{d \Omega_{6}}{\Omega_{3}^{2}-\Omega_{6}^{2}} \\
& K_{1}=\int \frac{d^{3} l}{(2 \pi)^{3}} \frac{a}{\Omega_{3}^{2}-\Omega_{5}^{2}} \cosh \left(\beta \Omega_{5}\right) \quad, \quad K_{2}=\int \frac{d^{3} l}{(2 \pi)^{3}} \frac{b}{\Omega_{3}^{2}-\Omega_{5}^{2}} \sinh \left(\beta \Omega_{5}\right) \\
& K_{3}=\int \frac{d^{3} l}{(2 \pi)^{3}} \frac{b \Omega_{5}}{\Omega_{4}^{2}-\Omega_{5}^{2}} \quad, J_{4}=\int \frac{d^{3} l}{(2 \pi)^{3}} \frac{d \Omega_{6}}{\Omega_{4}^{2}-\Omega_{6}^{2}} \cosh \left(\beta \Omega_{6}\right) \quad, \quad K_{4}=\int \frac{d^{3} l}{(2 \pi)^{3}} \frac{d}{\Omega_{3}^{2}-\Omega_{6}^{2}} \sinh \left(\beta \Omega_{6}\right)
\end{aligned}
$$

The integrals $L_{n}$ are obtained from the integrals $K_{n}$ by replacing $\Omega_{3}$ by $\Omega_{4}$.

$$
M_{1}=\int \frac{d^{3} l}{(2 \pi)^{3}} \frac{b \Omega_{5}}{\Omega_{3}^{2}-\Omega_{5}^{2}} \cosh \left(\beta \Omega_{5}\right) \quad, \quad M_{2}=\int \frac{d^{3} l}{(2 \pi)^{3}} \frac{a \Omega_{5}}{\Omega_{3}^{2}-\Omega_{5}^{2}} \sinh \left(\beta \Omega_{5}\right)
$$




$$
M_{3}=\int \frac{d^{3} l}{(2 \pi)^{3}} \frac{d \Omega_{6}}{\Omega_{3}^{2}-\Omega_{6}^{2}} \cosh \left(\beta \Omega_{6}\right) \quad, \quad M_{4}=\int \frac{d^{3} l}{(2 \pi)^{3}} \frac{c \Omega_{6}}{\Omega_{3}^{2}-\Omega_{6}^{2}} \sinh \left(\beta \Omega_{6}\right)
$$

The integrals $N_{n}$ are obtained from the integrals $M_{n}$ by replacing $\Omega_{3}$ by $\Omega_{4}$.

The expressions of sums of integrals in terms of $l_{i j}^{\Phi \Phi}$ are usefull :

$$
\begin{aligned}
I_{1}+I_{2}=V \int \frac{d^{3} l}{(2 \pi)^{3}} & \left\{-\frac{n_{\mathbf{1}+\mathbf{q}}+n_{\mathbf{1}}+1}{\Omega_{3}^{2}-\Omega_{5}^{2}}\left[\left(\frac{1}{\omega_{\mathbf{l}} \omega_{\mathbf{l}+\mathbf{q}}} l_{11}^{\Phi \Phi}+l_{22}^{\Phi \Phi}\right) \operatorname{coth}\left(\frac{\beta \Omega_{5}}{2}\right)+\frac{1}{\omega_{\mathbf{l}}} l_{12}^{\Phi \Phi}+\frac{1}{\omega_{\mathbf{1}+\mathbf{q}}} l_{21}^{\Phi \Phi}\right]\right. \\
& \left.+\frac{n_{\mathbf{1}+\mathbf{q}}-n_{\mathbf{1}}}{\Omega_{3}^{2}-\Omega_{6}^{2}}\left[\left(\frac{1}{\omega_{\mathbf{l}} \omega_{\mathbf{1}+\mathbf{q}}} l_{11}^{\Phi \Phi}-l_{22}^{\Phi \Phi}\right) \operatorname{coth}\left(\frac{\beta \Omega_{6}}{2}\right)+\frac{1}{\omega_{\mathbf{l}}} l_{12}^{\Phi \Phi}-\frac{1}{\omega_{\mathbf{1}+\mathbf{q}}} l_{21}^{\Phi \Phi}\right]\right\}
\end{aligned}
$$

$I_{3}+I_{4}$ is obtained from $I_{1}+I_{2}$ by replacing $\Omega_{3}$ by $\Omega_{4}$. The arguments of the functions $l_{i j}^{\Phi \Phi}$ in these integrals are $l_{i j}^{\Phi \Phi}\left(\mathbf{l},-\mathbf{q}-\mathbf{l}, \mathbf{p}_{1}, \mathbf{q}-\mathbf{p}_{1}, t^{\prime \prime}, t_{0}\right)$.

$$
\begin{aligned}
J_{1}+J_{2}= & V \int \frac{d^{3} l}{(2 \pi)^{3}} \times \\
& \frac{\Omega_{5}}{\Omega_{3}^{2}-\Omega_{5}^{2}}\left(n_{\mathbf{l}+\mathbf{q}}+n_{\mathbf{l}}+1\right)\left[\left(\frac{1}{\omega_{\mathbf{l}} \omega_{\mathbf{1}+\mathbf{q}}} l_{11}^{\Phi \Phi}+l_{22}^{\Phi \Phi}\right)+\left(\frac{1}{\omega_{\mathbf{l}}} l_{12}^{\Phi \Phi}+\frac{1}{\omega_{\mathbf{l}+\mathbf{q}}} l_{21}^{\Phi \Phi}\right) \operatorname{coth}\left(\frac{\beta \Omega_{5}}{2}\right)\right] \\
& -\frac{\Omega_{6}}{\Omega_{3}^{2}-\Omega_{6}^{2}}\left(n_{\mathbf{l}+\mathbf{q}}-n_{\mathbf{l}}\right)\left[\left(\frac{1}{\omega_{\mathbf{l}} \omega_{\mathbf{l}+\mathbf{q}}} l_{11}^{\Phi \Phi}-l_{22}^{\Phi \Phi}\right)+\left(\frac{1}{\omega_{\mathbf{l}}} l_{12}^{\Phi \Phi}-\frac{1}{\omega_{\mathbf{1}+\mathbf{q}}} l_{21}^{\Phi \Phi}\right) \operatorname{coth}\left(\frac{\beta \Omega_{6}}{2}\right)\right]
\end{aligned}
$$

$J_{3}+J_{4}$ is obtained from $J_{1}+J_{2}$ by replacing $\Omega_{3}$ by $\Omega_{4}$.

$$
\begin{aligned}
K_{1}+K_{2}+K_{3}+K_{4}= & -V \int \frac{d^{3} l}{(2 \pi)^{3}} \times \\
& \frac{n_{\mathbf{1}+\mathbf{q}}+n_{\mathbf{1}}+1}{\Omega_{3}^{2}-\Omega_{5}^{2}}\left[\left(\frac{1}{\omega_{\mathbf{1}} \omega_{\mathbf{1}+\mathbf{q}}} l_{11}^{\Phi \Phi}+l_{22}^{\Phi \Phi}\right) \operatorname{coth}\left(\frac{\beta \Omega_{5}}{2}\right)-\left(\frac{1}{\omega_{\mathbf{l}}} l_{12}^{\Phi \Phi}+\frac{1}{\omega_{\mathbf{1}+\mathbf{q}}} l_{21}^{\Phi \Phi}\right)\right] \\
& +\frac{n_{\mathbf{1}+\mathbf{q}}-n_{\mathbf{1}}}{\Omega_{3}^{2}-\Omega_{6}^{2}}\left[-\left(\frac{1}{\omega_{\mathbf{1}} \omega_{\mathbf{1}+\mathbf{q}}} l_{11}^{\Phi \Phi}-l_{22}^{\Phi \Phi}\right) \operatorname{coth}\left(\frac{\beta \Omega_{6}}{2}\right)+\frac{1}{\omega_{\mathbf{l}}} l_{12}^{\Phi \Phi}-\frac{1}{\omega_{\mathbf{1}+\mathbf{q}}} l_{21}^{\Phi \Phi}\right]
\end{aligned}
$$

$L_{1}+L_{2}+L_{3}+L_{4}$ is obtained from $K_{1}+K_{2}+K_{3}+K_{4}$ by replacing $\Omega_{3}$ by $\Omega_{4}$.

$$
\begin{aligned}
M_{1}+M_{2}+M_{3}+M_{4}= & -V \int \frac{d^{3} l}{(2 \pi)^{3}} \times \\
& \frac{\Omega_{5}}{\Omega_{3}^{2}-\Omega_{5}^{2}}\left(n_{\mathbf{l}+\mathbf{q}}+n_{\mathbf{1}}+1\right)\left[\left(\frac{1}{\omega_{\mathbf{1}} \omega_{\mathbf{1}+\mathbf{q}}} l_{11}^{\Phi \Phi}+l_{22}^{\Phi \Phi}\right)-\left(\frac{1}{\omega_{\mathbf{l}}} l_{12}^{\Phi \Phi}+\frac{1}{\omega_{\mathbf{1}+\mathbf{q}}} l_{21}^{\Phi \Phi}\right) \operatorname{coth}\left(\frac{\beta \Omega_{5}}{2}\right)\right] \\
& +\frac{\Omega_{6}}{\Omega_{3}^{2}-\Omega_{6}^{2}}\left(n_{\mathbf{l}+\mathbf{q}}-n_{\mathbf{1}}\right)\left[-\left(\frac{1}{\omega_{\mathbf{l}} \omega_{\mathbf{1}+\mathbf{q}}} l_{11}^{\Phi \Phi}-l_{22}^{\Phi \Phi}\right)+\left(\frac{1}{\omega_{\mathbf{l}}} l_{12}^{\Phi \Phi}-\frac{1}{\omega_{\mathbf{1}+\mathbf{q}}} l_{21}^{\Phi \Phi}\right) \operatorname{coth}\left(\frac{\beta \Omega_{6}}{2}\right)\right]
\end{aligned}
$$

$N_{1}+N_{2}+N_{3}+N_{4}$ is obtained from $M_{1}+M_{2}+M_{3}+M_{4}$ by replacing $\Omega_{3}$ by $\Omega_{4}$.

\section{Appendix F}


In this appendix, we give the solutions of the backward dynamical equations for $l_{i j}^{\Phi \Phi}$ at the first order. By noting :

$$
\begin{aligned}
& \Omega_{3}=\omega_{\mathbf{k}+\mathbf{q}}+\omega_{\mathbf{k}} \quad, \Omega_{4}=\omega_{\mathbf{k}+\mathbf{q}}-\omega_{\mathbf{k}} \\
& \Omega_{5}=\omega_{\mathbf{p}_{i}-\mathbf{q}}+\omega_{\mathbf{p}_{i}}, \Omega_{6}=\omega_{\mathbf{p}_{i}-\mathbf{q}}-\omega_{\mathbf{p}_{i}} \\
& F=\frac{b}{4} \frac{1}{\omega_{\mathbf{p}_{i}} \omega_{\mathbf{q}-\mathbf{p}_{i}}} \\
& \left.l_{11}^{\Phi \Phi}\left(\mathbf{k},-\mathbf{q}-\mathbf{k}, \mathbf{p}_{i},-\mathbf{p}_{i}+\mathbf{q}, t^{\prime}, t\right)\right)= \\
& -\frac{1}{2}\left[\delta^{3}\left(\mathbf{k}+\mathbf{p}_{i}\right)+\delta^{3}\left(-\mathbf{q}-\mathbf{k}+\mathbf{p}_{i}\right)\right] \cos \omega_{\mathbf{q}+\mathbf{k}}\left(t-t^{\prime}\right) \cos \omega_{\mathbf{k}}\left(t-t^{\prime}\right) \\
& -\frac{F}{2}\left(n_{\mathbf{q}-\mathbf{p}_{i}}+n_{\mathbf{p}_{i}}+1\right)\left[\frac{\Omega_{5}}{\Omega_{3}^{2}-\Omega_{5}^{2}} \cos \Omega_{3}\left(t-t^{\prime}\right)+\frac{\Omega_{5}}{\Omega_{4}^{2}-\Omega_{5}^{2}} \cos \Omega_{4}\left(t-t^{\prime}\right)\right] \\
& +\frac{F}{2}\left(n_{\mathbf{q}-\mathbf{p}_{i}}-n_{\mathbf{p}_{i}}\right)\left[\frac{\Omega_{6}}{\Omega_{3}^{2}-\Omega_{6}^{2}} \cos \Omega_{3}\left(t-t^{\prime}\right)+\frac{\Omega_{6}}{\Omega_{4}^{2}-\Omega_{6}^{2}} \cos \Omega_{4}\left(t-t^{\prime}\right)\right] \\
& +\frac{F}{2}\left(n_{\mathbf{q}-\mathbf{p}_{i}}+n_{\mathbf{p}_{i}}+1\right)\left(\frac{\Omega_{5}}{\Omega_{3}^{2}-\Omega_{5}^{2}}+\frac{\Omega_{5}}{\Omega_{4}^{2}-\Omega_{5}^{2}}\right) \cos \Omega_{5}\left(t-t^{\prime}\right) \\
& -\frac{F}{2}\left(n_{\mathbf{q}-\mathbf{p}_{i}}-n_{\mathbf{p}_{i}}\right)\left(\frac{\Omega_{6}}{\Omega_{3}^{2}-\Omega_{6}^{2}}+\frac{\Omega_{6}}{\Omega_{4}^{2}-\Omega_{6}^{2}}\right) \cos \Omega_{6}\left(t-t^{\prime}\right) \\
& \left.l_{12}^{\Phi \Phi}\left(\mathbf{k},-\mathbf{q}-\mathbf{k}, \mathbf{p}_{i},-\mathbf{p}_{i}+\mathbf{q}, t^{\prime}, t\right)\right)= \\
& -\frac{1}{2 i \omega_{\mathbf{k}+\mathbf{q}}}\left[\delta^{3}\left(\mathbf{k}+\mathbf{p}_{i}\right)+\delta^{3}\left(-\mathbf{q}-\mathbf{k}+\mathbf{p}_{i}\right)\right] \sin \omega_{\mathbf{q}+\mathbf{k}}\left(t-t^{\prime}\right) \cos \omega_{\mathbf{k}}\left(t-t^{\prime}\right) \\
& -\frac{F}{2 i \omega_{\mathbf{k}+\mathbf{q}}}\left(n_{\mathbf{q}-\mathbf{p}_{i}}+n_{\mathbf{p}_{i}}+1\right)\left[\frac{\Omega_{5}}{\Omega_{3}^{2}-\Omega_{5}^{2}} \sin \Omega_{3}\left(t-t^{\prime}\right)+\frac{\Omega_{5}}{\Omega_{4}^{2}-\Omega_{5}^{2}} \sin \Omega_{4}\left(t-t^{\prime}\right)\right] \\
& +\frac{F}{2 i \omega_{\mathbf{k}+\mathbf{q}}}\left(n_{\mathbf{q}-\mathbf{p}_{i}}-n_{\mathbf{p}_{i}}\right)\left[\frac{\Omega_{6}}{\Omega_{3}^{2}-\Omega_{6}^{2}} \sin \Omega_{3}\left(t-t^{\prime}\right)+\frac{\Omega_{6}}{\Omega_{4}^{2}-\Omega_{6}^{2}} \sin \Omega_{4}\left(t-t^{\prime}\right)\right] \\
& +\frac{F}{2 i \omega_{\mathbf{k}+\mathbf{q}}}\left(n_{\mathbf{q}-\mathbf{p}_{i}}+n_{\mathbf{p}_{i}}+1\right)\left(\frac{\Omega_{3}}{\Omega_{3}^{2}-\Omega_{5}^{2}}+\frac{\Omega_{4}}{\Omega_{4}^{2}-\Omega_{5}^{2}}\right) \sin \Omega_{5}\left(t-t^{\prime}\right) \\
& -\frac{F}{2 i \omega_{\mathbf{k}+\mathbf{q}}}\left(n_{\mathbf{q}-\mathbf{p}_{i}}-n_{\mathbf{p}_{i}}\right)\left(\frac{\Omega_{3}}{\Omega_{3}^{2}-\Omega_{6}^{2}}+\frac{\Omega_{4}}{\Omega_{4}^{2}-\Omega_{6}^{2}}\right) \sin \Omega_{6}\left(t-t^{\prime}\right) \\
& \left.l_{21}^{\Phi \Phi}\left(\mathbf{k},-\mathbf{q}-\mathbf{k}, \mathbf{p}_{i},-\mathbf{p}_{i}+\mathbf{q}, t^{\prime}, t\right)\right)= \\
& -\frac{1}{2 i \omega_{\mathbf{k}}}\left[\delta^{3}\left(\mathbf{k}+\mathbf{p}_{i}\right)+\delta^{3}\left(-\mathbf{q}-\mathbf{k}+\mathbf{p}_{i}\right)\right] \cos \omega_{\mathbf{q}+\mathbf{k}}\left(t-t^{\prime}\right) \sin \omega_{\mathbf{k}}\left(t-t^{\prime}\right) \\
& -\frac{F}{2 i \omega_{\mathbf{k}}}\left(n_{\mathbf{q}-\mathbf{p}_{i}}+n_{\mathbf{p}_{i}}+1\right)\left[\frac{\Omega_{5}}{\Omega_{3}^{2}-\Omega_{5}^{2}} \sin \Omega_{3}\left(t-t^{\prime}\right)-\frac{\Omega_{5}}{\Omega_{4}^{2}-\Omega_{5}^{2}} \sin \Omega_{4}\left(t-t^{\prime}\right)\right] \\
& +\frac{F}{2 i \omega_{\mathbf{k}}}\left(n_{\mathbf{q}-\mathbf{p}_{i}}-n_{\mathbf{p}_{i}}\right)\left[\frac{\Omega_{6}}{\Omega_{3}^{2}-\Omega_{6}^{2}} \sin \Omega_{3}\left(t-t^{\prime}\right)-\frac{\Omega_{6}}{\Omega_{4}^{2}-\Omega_{6}^{2}} \sin \Omega_{4}\left(t-t^{\prime}\right)\right] \\
& +\frac{F}{2 i \omega_{\mathbf{k}}}\left(n_{\mathbf{q}-\mathbf{p}_{i}}+n_{\mathbf{p}_{i}}+1\right)\left(\frac{\Omega_{3}}{\Omega_{3}^{2}-\Omega_{5}^{2}}-\frac{\Omega_{4}}{\Omega_{4}^{2}-\Omega_{5}^{2}}\right) \sin \Omega_{5}\left(t-t^{\prime}\right) \\
& -\frac{F}{2 i \omega_{\mathbf{k}}}\left(n_{\mathbf{q}-\mathbf{p}_{i}}-n_{\mathbf{p}_{i}}\right)\left(\frac{\Omega_{3}}{\Omega_{3}^{2}-\Omega_{6}^{2}}-\frac{\Omega_{4}}{\Omega_{4}^{2}-\Omega_{6}^{2}}\right) \sin \Omega_{6}\left(t-t^{\prime}\right)
\end{aligned}
$$




$$
\begin{aligned}
& \left.l_{22}^{\Phi \Phi}\left(\mathbf{k},-\mathbf{q}-\mathbf{k}, \mathbf{p}_{i},-\mathbf{p}_{i}+\mathbf{q}, t^{\prime}, t\right)\right)= \\
& +\frac{1}{2 \omega_{\mathbf{k}} \omega_{\mathbf{k}+\mathbf{q}}}\left[\delta^{3}\left(\mathbf{k}+\mathbf{p}_{i}\right)+\delta^{3}\left(-\mathbf{q}-\mathbf{k}+\mathbf{p}_{i}\right)\right] \sin \omega_{\mathbf{q}+\mathbf{k}}\left(t-t^{\prime}\right) \sin \omega_{\mathbf{k}}\left(t-t^{\prime}\right) \\
& -\frac{F}{2 \omega_{\mathbf{k}} \omega_{\mathbf{k}+\mathbf{q}}}\left(n_{\mathbf{q}-\mathbf{p}_{i}}+n_{\mathbf{p}_{i}}+1\right)\left[\frac{\Omega_{5}}{\Omega_{3}^{2}-\Omega_{5}^{2}} \cos \Omega_{3}\left(t-t^{\prime}\right)-\frac{\Omega_{5}}{\Omega_{4}^{2}-\Omega_{5}^{2}} \cos \Omega_{4}\left(t-t^{\prime}\right)\right] \\
& +\frac{F}{2 \omega_{\mathbf{k}} \omega_{\mathbf{k}+\mathbf{q}}}\left(n_{\mathbf{q}-\mathbf{p}_{i}}-n_{\mathbf{p}_{i}}\right)\left[\frac{\Omega_{6}}{\Omega_{3}^{2}-\Omega_{6}^{2}} \cos \Omega_{3}\left(t-t^{\prime}\right)-\frac{\Omega_{6}}{\Omega_{4}^{2}-\Omega_{6}^{2}} \cos \Omega_{4}\left(t-t^{\prime}\right)\right] \\
& +\frac{F}{2 \omega_{\mathbf{k}} \omega_{\mathbf{k}+\mathbf{q}}}\left(n_{\mathbf{q}-\mathbf{p}_{i}}+n_{\mathbf{p}_{i}}+1\right)\left(\frac{\Omega_{5}}{\Omega_{3}^{2}-\Omega_{5}^{2}}-\frac{\Omega_{5}}{\Omega_{4}^{2}-\Omega_{5}^{2}}\right) \cos \Omega_{5}\left(t-t^{\prime}\right) \\
& -\frac{F}{2 \omega_{\mathbf{k}} \omega_{\mathbf{k}+\mathbf{q}}}\left(n_{\mathbf{q}-\mathbf{p}_{i}}-n_{\mathbf{p}_{i}}\right)\left(\frac{\Omega_{6}}{\Omega_{3}^{2}-\Omega_{6}^{2}}-\frac{\Omega_{6}}{\Omega_{4}^{2}-\Omega_{6}^{2}}\right) \cos \Omega_{6}\left(t-t^{\prime}\right)
\end{aligned}
$$

\section{References}

[1] For reviews on inflationary cosmology, see : R. H. Brandenberger, Rev. Mod. Phys. 57, 1 (1985) ; A. Linde, in "Current Topics in Astrofundamental Physics, Proceedings of the Chalonge Erice School, edited by N. Sanchez and A. Zichichi, NATO ASI series C Vol 467, Kluwer Academic, Dordrecht, 1995.

[2] F. Cooper, Y. Kluger, E. Mottola and J. P. Paz, Phys. Rev. D51 (1995), 2377 ;

D. Boyanovsky, H. J. de Vega and R. Holman, Phys. Rev. D51 (1995), 734; D. Boyanovsky, F. Cooper, H. J. de Vega, P. Sodano, Phys. Rev. D 58 (1998) 025007.

[3] D. Yu. Grigoriev, V. A. Rubakov and M. E. Shaposhnikov, Nucl. Phys. B326 (1989), 737; D. Bödeker, Nucl. Phys. B486 (1997) 500

[4] P. Dirac, Proc. Cambridge Philos. Soc. 26 (1930) 736

[5] R. Jackiw and A. Kerman, Phys. Lett. A71 (1979) 158

[6] R. Balian and M. Vénéroni, Nucl. Phys. B408 (1993) 445

[7] J. Schwinger, J. Math. Phys. 2 (1961) 407; L. V. Keldish, JETP 20 (1965) 1018

[8] N. P. Landsmann and C. G. van Weert, Phys. Rep. 145 (1987) 141

[9] O. Eboli, R. Jackiw and S. Y. Pi, Phys. Rev. D37 (1988) 3557

[10] C. Martin, Ann. of Phys. 271 (1999) 294

[11] C. Martin, Phys. Rev. D52 (1995) 7121 
[12] J. Zinn-Justin, "Quantum field theory and critical phenomena", The international series of monographs on physics, Oxford University Press, 1990.

[13] E. Gerjuoy, A. R. P. Rau and L. Spruch, Rev. Mod. Phys. 55 (1983), 725

[14] M. Benarous, Phd Thesis (University of Paris-Sud, Orsay, 1991)

[15] R. Balian and M. Vénéroni, Ann. of Phys. 164 (1985) 334

[16] A. K. Kerman and C. Y. Lin, Ann. of Phys. 241 (1995) 185; Ann. of Phys. 269 (1998) 55

[17] G. Aarts and J. Smit, Nucl. Phys. B511 (1993) 4991

[18] L. Dolan and R. Jackiw, Phys. Rev. D9 (1995) 185

[19] P.Bonche and H. Flocard, Nucl. Phys. A437 (1987) 301.

[20] G. Parisi, Statistical Field Theory (Addison-Weysley Publishing Compagny, 1988)

[21] E. Wang and U. Heinz, Phys. Rev. D53 (1996), 5978; A. Jakovac, hep-th/9808349

[22] D. Boyanovsky, H. J. de Vega, R. Holman, S. Prem Kumar and R. D. Pisarski, Phys. Rev. D58 125009 